\title{
Proceedings of the Inaugural International Conference on Obesity and Chronic Diseases (ICOCD-2016)
}

\section{$\underline{\text { Keynote Presentations }}$}

\section{Obesity and Cancer Risk: The Gut Microbiota Connection}

\author{
Reza Hakkak $^{1-3^{*}}$, Soheila Korourian ${ }^{4}$, Steven Foley ${ }^{5}$ and Bruce Erickson ${ }^{5}$ \\ ${ }^{1}$ Department of Dietetics and Nutrition, University of Arkansas for Medical Sciences, AR, USA \\ ${ }^{2}$ Arkansas Children Hospital Research Institute, AR, USA \\ ${ }^{3}$ Department of Pediatrics, University of Arkansas for Medical Sciences, AR, USA \\ ${ }^{4}$ Department of Pathology, University of Arkansas for Medical Sciences, AR, USA \\ ${ }^{5}$ National Center for Toxicological Research, U.S. Food and Drug Administration, AR, USA
}

\begin{abstract}
The rate of overweight and obese adults continues to rise in the US and world. Obese women have higher risk and mortality rates from all types of cancers including breast cancer. The intestinal microbiota is composed of a diverse population of obligate and facultative anaerobic microorganisms, and these organisms carry out a broad range of metabolic activities. Previously, we reported that a soy protein diet containing high isoflavone levels promoted 7,12 dimethylbenz [a] anthracene (DMBA) induced mammary tumor development compared to a casein-based diet and the plasma equol was significantly lower in obese soy-fed rats compared to lean soy-fed rats. Obesity has been linked to changes in the intestinal microbiota, but the effects of obesity and soy protein diet on the composition of the intestinal microbiota have not been studied. The objective of study was to determine the effects of obesity and diet containing soy protein isolate on gut microbiota. Lean and obese female Zucker rats $(n=34)$ were randomly assigned to 1) casein, or 2) soy protein diets for 8 weeks. Fecal samples, collected at the end of the experiment, were analyzed using $16 \mathrm{~S}$ rRNA gene sequencing to determine the bacterial populations present. Bacterial DNA was isolated from the fecal samples using a commercial kit and the DNA prepared for sequencing using barcoded primers specific for the Illumina MiSeq platform. Following sequencing, the readings were de-multiplexed and the different taxa identified to characterize the total bacterial populations. Our results suggest that there are differences in gut microbiota associated with diet and the lean/ obese state. Further investigation will be needed to determine the effects of obesity and soy protein diet on gut microbiota in relation to DMBA-induced mammary tumor formation.
\end{abstract}

\section{$\mathrm{Mg}^{2+}$-Deficiency in Liver Cells: At the Cross-Road Between Inflammation and Dysmetabolism}

\author{
Andrea Romani*, Chesinta Voma and Lauren Keenan \\ Case Western Reserve University, Department of Physiology and Biophysics, Cleveland, OH, USA
}

\begin{abstract}
A decrease in tissue and serum $\mathrm{Mg}^{2+}$ content has been observed in several endocrinopathies including metabolic syndrome and diabetes, but it is still undefined whether $\mathrm{Mg}^{2+}$ deficiency plays a role in the onset of these pathologies and/or their complications. Our experimental observation in animals and human liver cells indicate that $\mathrm{Mg}^{2+}$ deficiency increases G6P entry into the ER, and results in an increased oxidation by H6PD. The produced NADPH is then utilized by the 11ß-HSD1 to convert inactive cortisone to active cortisol. Our data indicate that cortisol production is markedly increased in $\mathrm{Mg}^{2+} \mathrm{deficient}$ hepatocytes and results in enhanced gluconeogenesis, hepatic fatty acid synthesis, and intrahepatic triglycerides deposition. Furthermore, $\mathrm{Mg}^{2+}$-deficient hepatocytes present decreased insulin responsiveness, which is further compromised by cortisol
\end{abstract}


production. Returning cellular $\mathrm{Mg}^{2+}$ content to physiological levels dramatically decreases cortisol production, and progressively renormalizes expression and activity of H6P, $11 \beta-\mathrm{HSD} 1$, and cortisol-responsive genes. Investigation into the mechanism responsible for $11 \beta-H S D 1$ increased expression suggest the involvement of increased NFkB translocation to the nucleus and consequently enhanced IL-1 $\alpha$ and TNF expression in the process. Taken together, our results suggest that $\mathrm{Mg}^{2+}$ deficiency precedes the onset of metabolic syndrome, setting the conditions for an increased intrahepatic production of cortisol and a decreased insulin responsiveness by acting at multiple levels including NFkB translocation, and H6PD and 11 $\beta-H S D 1$ activity and expression while providing a constant entry of G6P into the ER to support the activity of the latter enzymes.

\title{
Transcriptomic Identification of a Novel Obesity Gene
}

\section{Christopher Jenkinson}

South Texas Diabetes and Obesity Institute (STDOI), University of Texas Rio Grande Valley (UTRGV), TX, USA

\begin{abstract}
Obesity is a complex disorder that is highly prevalent in Mexican Americans, and is strongly associated with insulin resistance $(\mathrm{OB} / \mathrm{IR})$. We measured genome-wide gene expression in adipose tissue from 75 unrelated Mexican Americans to detect gene expression associated with OB/IR. Fasting RNA was extracted from adipose biopsies, and gene expression data generated using Illumina Bead Arrays (47k probes). Gene sets analysis identified one gene, alcohol dehydrogenase 1B (ADH1B) with significantly enriched expression $(\mathrm{P}<10-60)$ as a candidate for involvement in relevant metabolic pathways. Adipocyte ADH1B was highly and specifically expressed in adipose tissue at levels greater than in all other tissues examined. $\mathrm{ADH} 1 \mathrm{~B}$ expression was inversely correlated with all $\mathrm{OB} / \mathrm{IR}$ variables measured: waist circumference $\left(\mathrm{P}=2.8 \times 10^{-9}\right), \mathrm{BMI}$ $(\mathrm{P}<0.0001)$, FPI $(\mathrm{P}<0.001)$, HOMA-IR $(\mathrm{P}<0.01)$, Matsuda Index $(\mathrm{P}<0.01)$, $\beta$-cell function (oral disposition index across 120 minutes of the OGTT (ODI1-120; OGTT) $\left(\mathrm{P}=8.6 \times 10^{-4}\right)$, pre-T2D (impaired glucose tolerance and fasting glucose during the OGTT $\left(\mathrm{P}=8.6 \times 10^{-5}\right)$; and, from the insulin clamp: total glucose disposal $\left(\mathrm{P}=8.6 \times 10^{-10}\right)$, hepatic glucose production (hGP, $\mathrm{P}=8.6 \times 10^{-4}$ ) free fatty acid IR index (FFA-IRI; $\mathrm{P}=1.3 \times 10^{-4}$ ). The TGD rate represents mainly skeletal muscle IR; hGP indicates hepatic IR and FFA IR indicate adipose IR. ADH1B protein was decreased with high BMI $(\mathrm{P}<$ $0.05, \mathrm{~N}=12$ ). Decreased adipose $\mathrm{ADH} 1 \mathrm{~B}$ expression was associated with increased obesity, whole body IR, IR in liver, skeletal muscle and adipose tissue, and with declining $\beta$-cell function. Interestingly, serum levels of circulating endogenous ethanol, a substrate for $\mathrm{ADH} 1 \mathrm{~B}$, decreased with increasing $\mathrm{OB} / \mathrm{IR}(\mathrm{P}=0.019$ with a 7 -fold increase in $\mathrm{FPI})$.
\end{abstract}

\section{$\underline{\text { Special Session I }}$}

\section{Nutrition and Obesity Grant Opportunities Through USDA and NIH}

\author{
Deirdra N. Chester ${ }^{*}$ and Tanya Agurs-Collins ${ }^{2^{*}}$ \\ ${ }^{1}$ Division of Nutrition, Institute of Food Safety and Nutrition, National Institute of Food and Agriculture, USDA, MD, USA \\ ${ }^{2}$ Health Behaviors Research Branch, Division of Cancer Control and Population Sciences, National Cancer Institute/NIH/DHHS, \\ $M D, U S A$
}

\begin{abstract}
The Childhood Obesity Prevention Program focuses on the societal challenge to end child obesity. Specific program areas are designed to achieve the long-term outcome of reducing the prevalence of overweight and obesity among children and adolescents. The Division of Nutrition (DN) supports research on the societal challenge to end child obesity. The focus is on the generation of new knowledge about the behavioral, social, cultural, and environmental factors that influence excessive weight gain in children and adolescents. DN is with the Institute of Food Safety and Nutrition (IFSN) at USDA - National Institute of Food and Agriculture (NIFA). Considerable evidence indicates that factors related to energy balance - the integrated effects of diet, physical activity, and genetics on growth and body weight - are known risk factors for many chronic diseases and conditions, such as several forms of cancer and cardiovascular disease. Recent systematic reviews and meta-analyses of observational studies suggest that being overweight or obese increases risk for initial cancers, recurrence, and second cancers and decreases overall survival among cancer survivors. These results also suggest that physical activity decreases cancer risk and mortality. Interest is rapidly increasing in the role sedentary behavior as a unique disease risk factor. The Health Behaviors Research Branch (HBRB) supports research on cancer prevention behaviors and outcomes, which includes diet, physical activity, sedentary behavior, energy balance, obesity and genetic influences on behaviors. HBRB is within the National Cancer Institute's (NCI) Behavioral Research Program (BRP), in the Division of Cancer Control and Population Sciences (DCCPS).
\end{abstract}




\title{
Featured Presentations
}

\section{Prevention of Excess Weight Gain in Pediatric Primary Care: Beverages Only or Multiple Life Style Factors. The Smart Step Study, a Cluster-randomized Clinical Trial}

\author{
Nicolas Stettler-Davis
}

The Lewin Group, VA, USA

\begin{abstract}
Background: Insufficient evidence exists to support obesity prevention in pediatric primary care.

Objectives: To test a theory-based behavior modification intervention delivered by trained pediatric primary care providers for obesity prevention.

Methods: Efficacy trial with cluster randomization (practice level) and a 12-session 12-month sweetened beverages decrease intervention or a comprehensive dietary and physical activity intervention, compared with a control intervention unrelated to obesity among children ages $8-12$ years.

Results: A low practice recruitment rate was observed. Among participants, the increase in body mass index z-score (BMIz) for the 139 subjects (11 practices) randomized to any of the two obesity interventions (combined group) was less than that of the 33 subjects (five practices) randomized to the control intervention $(-0.089,95 \%$ confidence interval [CI]: -0.170 to -0.008 , $\mathrm{P}=0.03)$ with a $-1.44 \mathrm{~kg}$ weight difference $(95 \% \mathrm{CI}:-2.98$ to $+0.10 \mathrm{~kg}, \mathrm{P}=0.095)$. The incidences of obesity and excess weight gain were lower in the obesity interventions, but the number of subjects was small. Post hoc analyses comparing the beverage only to the control intervention also showed an intervention benefit on BMIz $(-0.083,95 \% \mathrm{CI}:-0.165$ to $-0.001, \mathrm{P}=0.048)$.

Conclusions: For participating families, an obesity prevention intervention delivered by pediatric primary care clinicians, who are compensated, trained and continuously supported by behavioral specialists, can impact children's BMIz. This study also provides additional data supporting a causal link between sweetened beverages and excess weight gain in children.
\end{abstract}

\section{Overfed and Undernourished in the Middle East: Obesity is Associated with Micronutrient Deficiencies and Food Insecurity}

\author{
Nahla Hwalla \\ American University of Beirut, Beirut, Lebanon
}

\begin{abstract}
The Middle Eastern region has been witnessing a triple burden of disease (coexistence of undernutrition, micronutrient deficiencies, and overweight/obesity) as a consequence of nutrition transition experienced by countries in the region. The association between over-nutrition and under-nutrition in these countries has not been investigated to date. Obese subjects are considered over-nourished, whereas under-nourished individuals are considered stunted and/or micronutrient deficient. However, analyzing recent data from these countries revealed the coexistence of micronutrient deficiencies and food insecurity both in the under-nourished and the obese. Available data from Middle Eastern countries were analyzed for prevalence of obesity, stunting, and micronutrient deficiencies among children, and where available, the magnitude of micronutrient deficiencies in this population group. Data showed that high-income countries of the region have high obesity rates concomitant with high prevalence of micronutrient deficiencies. Under-five Saudi children suffer from high prevalence of iron and iodine deficiencies (14.5\% and 23\%, respectively), as well as anemia (41.4\%), despite having the highest obesity rate in the region (10\%). A showcase of the coexistence of micronutrient deficiencies and obesity were found in Lebanon where vitamin D and magnesium deficiencies were highly prevalent in $97 \%$ and $87 \%$ of obese children and adolescents (6-19 years), respectively. In addition, Lebanese children and adolescents ( $4-18$ years) from food insecure households were significantly more overweight/obese than those from food secure households ( $16.8 \%$ vs. $9.7 \% ; \mathrm{p}<0.05)$. In conclusion, cohort studies are needed to adequately determine early-life culturally-related feeding practices and their future impact on the triple burden of disease in the region.
\end{abstract}




\title{
Failure to Address Overweight and Obesity in Children with Autism Spectrum Disorders: What's the Impact of Doing Nothing?
}

\author{
Tina Crook \\ Department of Dietetics and Nutrition, University of Arkansas for Medical Sciences, AR, USA
}

\begin{abstract}
Data from the 2011-2012 National Health and Nutrition Examination Survey indicate 32\% of children in the U.S. are overweight or obese, making excess weight the most predominant health risk in the pediatric population. Current research indicates that children with autism spectrum disorders (ASDs) may be at even greater risk of obesity than the general pediatric population. Factors cited in the literature that may be associated with increased obesity risk in children with ASDs include 1) limited food repertoire that emphasizes high carbohydrate, energy dense foods; 2) preference for sedentary activities; 3 ) use of psychotropic medications; 4) sleep disorders and 5) genetics. While recommendations were established in 2007 by an Expert Committee that included "Universal Assessment" (UA) of all children for obesity risk by primary care providers, barriers may exist inhibiting its application. This appears to be true especially in children with ASDs, perhaps because other problems take priority (such as communication and behavior issues) and clinicians and caregivers may not relate weight to long-term outcomes. It is imperative that healthcare providers are educated about the process of identifying, assessing and treating obesity in all children, including those with ASDs. Furthermore, healthcare providers must rank weight management among the top priorities when assessing children with ASDs since co-morbidities may develop if weight issues are not addressed. The presentation will explore the projected impact of not addressing weight, barriers to interventions and strategies for overcoming weight management obstacles in children with ASDs.
\end{abstract}

\section{Risk Habits Associated to Overweight (OW) and Obesity (OB) in Adolescents of a Secondary School}

\author{
Ana Lilia Rodriguez Ventura*, Arturo Parra Solano, Minerva Hernandez, Laura Almaguer, Carolina Paredes, Reyna \\ Samano, Bernarda Sanchez, Rosa Martinez and Maricruz Hdez
}

Instituto Nacional de Perinatologia, Mexico

\begin{abstract}
Introduction: In spite of the worldwide epidemic of overweight and obesity and the international recommendations focus on the behavior, habits risk for overweight and obesity, few studies reporting such habits and their association with other anthropometric and metabolic variables.

Methods and Patients: Cross-sectional study of 105 adolescents of a secondary school in order to identify nutritional and metabolic status and risk habits associated to OW/OB. Previous standardized of nutritionists, they recorded weight, height, waist; blood pressure, fat percentage, frequency of food, 24 hour recalls and risk habits. After 12 hours fasting, a blood venous sample was taken previous signed informed letter by adolescents and parents or tutors.

Results: The $67.6 \%$ was female sex, $42.9 \%$ had low socioeconomic level; $83.3 \%$ ate only $0-1$ equivalent of vegetables per day, $62.7 \%$ only $0-1$ equivalent of fruits per day, $60 \%$ did not exercise or it was not enough $<2 \mathrm{hrs}$ per week, $64 \%$ watched TV $>2 \mathrm{hrs} /$ day, $51 \%$ sleep < 9 hrs. The media for age was $13.31 \pm 0.67$ years, weight $53.9 \pm 12.2 \mathrm{~kg}$, body mass index $(\mathrm{BMI}) 22.4 \pm 4.3 \mathrm{~kg} / \mathrm{m}^{2}, \mathrm{waist}$ $73.6 \pm 13.4 \mathrm{~cm}$, fat percentage $30.15 \pm 9.2 \%$, systolic blood pressure $114.2+7.6 \mathrm{mmHg}$, diastolic $72.6 \pm 7.4$; cholesterol $177.2 \pm$ $44.1 \mathrm{mg} / \mathrm{dl}$, triglycerides $98.8 \pm 52 \mathrm{mg} / \mathrm{dl}$, glucose $87.5 \pm 8.7 \mathrm{mg} / \mathrm{dl}$, HDL $51.9 \pm 12.8 \mathrm{mg} / \mathrm{dl}$. Media of calories consumed was $1427 \pm 623 ; 18.4 \%$ was overweight and $31.1 \%$ obesity according CDC tables. Using Pearson correlation, a higher weight, lower calories, more prolonged fasting, less hours of sleep, higher measures of waist, blood pressure, fat percentage and waist/height index (WHI) and only higher WHI was associated to higher triglycerides concentrations.

Conclusions: Almost all adolescents, independently of nutritional status presented risk habits to develop OW or OB, especially sleep short and less calories associated with prolonged fasting. WHI was a better marker of hypertriglyceridemia. It is necessary to promote better habits -a healthy lifestyle- across the population.
\end{abstract}




\title{
TheEmerging ChronicDisease:EducatingProviders to Start the Difficult Conversations
}

\author{
Susan Farus-Brown \\ Obio University School of Nursing, OH, USA
}

\begin{abstract}
The objective of this project was to provide education on utilization of a tool kit to increase the nurse practitioner (NP) participants' knowledge base in regards to pediatric obesity prevention, recognition, and treatment. As the pre-intervention, a questionnaire was distributed to the participants to measure their knowledge of childhood obesity. An educational session was provided and then the participants utilized a tool kit in their clinical practice. After a two-month period the Project Director met with the participants and distributed the same questionnaire to determine if participant knowledge, skills and perceptions changed after adopting the tool kit principles into their practice. Data analysis was completed utilizing an analysis of both the mode and median for each question to determine whether or not there was a change in the pre and post-intervention responses. An analysis of pre and post-frequency distributions was completed to determine the direction and extent of that change. Statistical significance of each change was tested utilizing the Mann-Whitney U-Test. Mode, median and statistical significance were calculated utilizing Excel. Post-intervention participants reported that children would not outgrow being overweight, and identified that a significant barrier to pediatric obesity treatment was not the patient, but lack of preparation on the part of NPs. The results supported that increased knowledge did translate into improved treatment practices in the ambulatory care setting. As more NPs are prepared to recognize, prevent, and treat pediatric obesity, the health of our children will improve significantly.
\end{abstract}

\section{Childhood Obesity Among Intellectually Disable Children in Hong Kong: A Cross- Sectional Study}

\author{
Yang Gao, Ya-jun Huang, Health Kwok and Ada Chan \\ Hong Kong Baptist University, Hong Kong, China
}

\begin{abstract}
Aim: This cross-sectional study aims at investigating the epidemic characteristics of obesity in children with mild and moderate intellectual disability (ID) in Hong Kong.

Methods: The study was conducted in 12 special schools from June to November, 2015. Parents of participants completed a self-administered questionnaire on children's eating habits and physical activity (PA), parenting style, and parents' height and weight. Children's height and weight were measured at school following standard procedures. Overweight and obesity were defined using the Cole's classification criteria (Cole, 2000). Univariate and multivariate regression were performed to estimate the relationship between potential risk factors and childhood obesity.
\end{abstract}

Results: Out of 558 participants (age: 6-21 years, $12.2 \pm 3.9$ years; boys: 68.8\%), 31.3\% were overweight or obese, higher than their general counterparts in Hong Kong (20.3\%). Multivariate regression revealed that children spending more time in moderate-to-vigorous PA (MVPA), those taking more meats, those taking more fried food, and those with parental overweight and obesity were more likely to be overweight or obese (adjusted ORs: 1.69-5.24, $\mathrm{P}<0.05$ ), whilst those with parents' positive attitude towards children's weight management was less likely to be overweight or obese (adjusted OR: 0.46, P =0.028).

Conclusions: Children with ID are more vulnerable to obesity. Parents may have already taken some actions against children's obesity, especially by increasing PA level and intake of fruits and vegetable. However, large portion of high-energy food may play a more important role. There is an urgent call for new effective interventions for obesity among the special children.

\section{KidsJust WannaHave Fun: Children’s Experiences of Weight Management Programme}

\author{
Libby Watson
}

University of East London, United Kingdom

\section{Abstract}

This research is the first qualitative study that explored children's accounts of their experiences of the UK's largest childhood 
obesity programme, MEND (Mind, Exercise, Nutrition. . .Do it!), including the vital post-treatment maintenance period (at least one year since completing). This study also explored the under-researched phase of adolescence: whilst multi-component family interventions are recommended for tackling childhood obesity, the participants in this study were at a developmental stage characterized by peer influences. Participants were also from diverse cultural backgrounds. Findings unearthed an unexpected and strong theme - that of fun - the focus of the above-titled journal article. Optimizing conditions for fun is integral to children's (and adults') engagement with, and maintenance of, healthy lifestyle activities. Additional themes that arose from the complete analysis (L. Watson, Unpublished doctoral thesis) will also be presented. As a practicing Clinical Psychologist, Dr. Libby Watson will also present practice-based evidence and insights from her experience working in adult weight management and chronic disease settings within the United Kingdom's National Health Service, providing key links between research findings and theories to their real world applications for clinical practice [1].

\title{
Reference
}

1. Watson L, Baker M, Chadwick P. 2016. Kids just wanna have fun: children's experiences of a weight management programme. British Journal of Health Psychology 21(2): 407-420. doi: 10.1111/bjhp.12175

\section{Relationship Between Socioeconomic Status and Obesity in Mexican Children and Adolescents}

\author{
Michelle M. Haby ${ }^{*}$, Rolando G. Diaz Zavala ${ }^{1}$, Mauro E. Valencia ${ }^{1}$ and Anna Peeters ${ }^{2}$ \\ ${ }^{1}$ Department of Chemical and Biological Sciences, University of Sonora, Mexico \\ ${ }^{2}$ School of Health and Social Development, Deakin University, Australia
}

\begin{abstract}
In developed countries, obesity affects people of lower socioeconomic status (SES) more than those of higher SES, and this is the case for both children and adults. However, in developing countries the relationship is different. In low-income countries, obesity is more prevalent in people of higher SES and less prevalent in people of lower SES. In middle-income countries, there is ongoing debate and research as to whether obesity primarily affects the poor or the rich. Recent studies in Brazil (a middleincome country) suggest that obesity prevalence is decreasing amongst women with higher income and increasing in women with lower income. We hypothesize that, with the nutrition transition and associated change in dietary habits and physical activity, a similar change will be found in children and adolescents in Mexico. We are analyzing the Mexican National Health and Nutrition Surveys (ENSANUT) from 2000, 2006 and 2012 to determine the relationship between obesity and SES and changes over time in children and adolescents. Outcome variables include BMI z-score and obesity prevalence, calculated using the International Obesity Task Force age- and sex- specific BMI cut-offs. SES variables include a composite variable of SES and educational level of parents. Results will be presented at the conference. Understanding the differences between subgroups of the population is critical to ensuring policy makers can make informed decisions as to where preventive efforts should be focused. This is particularly important in light of evidence that demonstrates differential effectiveness of a number of obesity prevention interventions according to SES.
\end{abstract}

\section{Cost-Effectiveness of an Intervention in UK Pre-School Children}

\author{
Gerry Richardson \\ Centre for Health Economics, University of York, UK
}

\begin{abstract}
Background: In the United Kingdom, obesity prevalence in childhood remains high and levels of physical activity remain low. These related childhood influences often impact on both behavior and health status as adults. However, there is a lack of feasible, evidence-based approaches to reduce obesity and improve physical activity in infancy. Interventions with parents and young children have the potential to improve these childhood outcomes with the expectation that health benefits will be achieved not only in childhood but also into adulthood; there is also the potential to improve parental outcomes. There are few pre-schools, playground-based interventions and these have given inconclusive results. This study aimed to assess the cost effectiveness of an outdoor playground-based physical activity intervention for parents and their children aged from 18 months to 4 years (Preschoolers in the Playground or PiP). We will also consider other UK evaluations (such as the Healthy and Active Parenting Programme for Early Years) that aim to reduce obesity and improve physical activity levels in children.
\end{abstract}

Methods: This Cost Effectiveness Analysis (CEA) is based on data from a Randomized Controlled Trial conducted in 
a deprived UK population comparing PiP with usual practice and makes an assessment of the value for money of the PiP intervention. The pilot trial is set in Bradford, UK; 164 children participated in the study. The CEA is conducted from the perspective of the UK National Health Service (NHS) and personal social services and uses 2013/14 prices. Health Related Quality of Life (HRQoL) is assessed for both parents and children using Quality Adjusted Life Years (QALYs) and Pediatric Quality of Life Inventory (PedsQL) respectively.

Results: Parents HRQoL improved by 0.058 QALYs (95\% CI -0.009 to 0.125 ) at an additional cost of $£ 1173$ (-£903 to $£ 3,250)$ per participant generating an incremental cost-effectiveness ratio of $£ 20,215$; there is a large degree of uncertainty around the results. Children in the intervention group had an improved HRQoL over the trial period (increase of $1.5 \mathrm{PedsQL}$ points) while the control group showed a reduced HRQoL (decrease of 0.2). These results are preliminary.

Conclusion: The PiP intervention is potentially cost-effective but the degree of uncertainty around the results means that further studies and analysis would be informative. Other interventions in young children and their parents have the potential to offer value for money and need to be considered.

\title{
$\underline{\text { Special Session II }}$
}

\section{Molecular Medicine and Functional Genomics of Energy Metabolism and Chronic Diseases}

\author{
Hiroaki Tanaka \\ Fukuoka University Institute for Physical Activity, Japan
}

\begin{abstract}
This presentation will address the studies concerning the effects of running at lactate threshold (LT), so-called slow jogging. Supervised physical training performed at LT could induce the improvement in VO2max, thigh muscle mass, lipid profiles, insulin sensitivity and abdominal fat; without diet. Based on these studies, we designed health tourism program for obese people for one week with slow jogging and dieting inducing the large energy deficit $>2000 \mathrm{kcal} / \mathrm{d}$. Subjects succeeded in $3.2 \%$ weight loss with improving lipid profiles similar to the prolonged statin treatment.
\end{abstract}

\section{Electric Pulse Stimulation (ESP)/Mechanical Stretch as a Model for Physical Exercise and Chronic Diseases using Cell Cultured Myotubes}

\author{
Makoto Kanzaki \\ Graduate School of Biomedical Engineering, Tohoku University, Japan
}

\begin{abstract}
Physical exercise provokes a highly complex series of interrelated stimuli, including mechanical stress, acute energy expenditure, reactive oxidative stress, and others in working skeletal muscles, which is well known to be able to subsequently deliver many beneficial effects for maintaining better health. Understanding of molecular mechanisms underlying such beneficial effects provide us to allow new therapeutic approaches for preventing and/or treating various lifestyle-related diseases including type 2 diabetes, atherosclerosis, stroke and obesity. A model cell culture system that faithfully mimics the features of in vivo muscle would be an invaluable tool for investigating a wide array of biological phenomena including muscle development, hypertrophy and atrophy, and would also be applicable to drug screening. However, there was no such muscle cell culture model system which can be utilized for functionally-active muscle research, because conventional muscle cell culture systems do not produce myotubes with vigorous contractile activity. Recently, we succeeded in establishing an "Electric Pulse Stimulation (EPS)-evoked myotube contraction culture system (in vitro Exercise model)" which generates highly-developed cultured myotubes similar to in vivo skeletal muscle. Importantly, the highly-developed contractile myotubes are not only with [1] vigorous contractile ability resulting from de novo sarcomere structure [1], but also with higher functional properties in terms of [2] contraction-induced myokines secretion, [3] higher energy expenditure along with activation of various stress-response kinases, [4] a fiber-type switching in myosin heavy chain (MHC) expression of "fast-to-slow" type, and [5] better insulin responsiveness [2-4]. Given the highly complex interrelated stimuli evoked by muscle contractile activity, physiological responses of working muscle to physical exercise have been assessed using animal models and human subjects. Although it is quite difficult to directly compare in vivo exercise (muscle contraction) and EPS-evoked contraction of cultured myotubes, and caution must
\end{abstract}


be exercised in interpreting the results in some cases, our careful investigations as well as recent related publications from other researchers5 provide compelling evidence demonstrating EPS-evoked changes in "cultured" contractile myotubes to be involved in the physiological process of muscle maturation in terms of contractility and subsequent alterations in gene expressions and metabolic properties that are observable in skeletal muscles in vivo. Thus, "in vitro Exercise model" would have great usefulness for studying a broad spectrum of skeletal muscle research area that was formerly completely dependent on animal experiments because of their fundamental characteristics including vigorous contractile activity.

\title{
References
}

1. Fujita H, Nedachi T, Kanzaki M. 2007. Accelerated de novo sarcomere assembly by electric pulse stimulation in C2C12 myotubes. Exp Cell Res 313(9): 1853-1865.

2. Nedachi T, Fujita H, Kanzaki M. 2008. Contractile C2C12 myotube model for studying exercise-inducible responses in skeletal muscle. Am J Physiol EndocrinolMetab 295(5): E1191-1204.

3. Nedachi T, Hatakeyama H, Kono T, Sato M, Kanzaki M. 2009. Characterization of contraction-inducible CXC chemokines and their roles in C2C12 myocytes. Am J Physiol Endocrinol Metab 297(4): E866-878.

4. Farmawati A, Kitajima Y, Nedachi T, Sato M, Kanzaki M, et al. 2013. Characterization of contraction-induced IL-6 up-regulation using contractile C2C12 myotubes. Endocr J60(2): 137-147.

5. Brown AE, Jones DE, Walker M, Newton JL. 2015. Abnormalities of AMPK activation and glucose uptake in cultured skeletal muscle cells from individuals with chronic fatigue syndrome. PLoS One10(4): e0122982.

\section{Functional Genomic and Physical Exercise Studies Potentially Leading to the Development of Obesity and Sedentary Related Diseases Drugs Discovery}

\author{
Yoshioka $\mathbf{M}^{1,2^{*}}$, Melouane $\mathrm{A}^{1,2}$ and St-Amand $\mathrm{J}^{1,2}$ \\ ${ }^{1}$ Functional Genomics Laboratory, Endocrinology and Nephrology, CHUQ-CHUL Research Center, Canada \\ ${ }^{2}$ Department of Molecular Medicine, University Laval, Canada
}

\begin{abstract}
Moderate-intensity endurance exercise at the Lactate Threshold (LT) can be one of the best treatments for metabolic syndrome, obesity and sedentary related diseases, such as sarcopenia. However, some individuals such as obese, elderly and cardiac patients cannot or does not want to perform recommended physical exercise to have health benefits. Thus, the aim of our research projects is to develop exercise pill or surrogate for such individuals using functional genomic studies in order to identify candidate transcripts specifically regulated in elderly individuals after LT training. According to the current obesity epidemics and its associations with complex diseases, the studies focusing to develop surrogate to exercise therapy are thus urgently needed. We have characterized, for the first time, the transcriptomes of several tissues involved in energy intake and expenditure as well as their regulation by several factors, such as exercise, high fat diet and steroid hormones, which influence energy homeostasis and body fat distribution. We have compared sedentary individuals with sprinters and endurance athletes, using the powerful Serial Analysis of Gene Expression (SAGE) method. Among all the genes expressed in skeletal muscle, we have identified several hundreds of transcripts differentially regulated. Very interestingly, several no match tags were not expressed at all in normal individuals; however, they were expressed hundreds of times in endurance athletes. We have called these potential novel genes: endurance athlete genes. Then, we have also performed the same type of studies after LT training and detraining in young adults and elderly individuals. In order to further characterize the roles of the key genes in LT training adaptations, development of obesity and related diseases, we have made a selection of the novel candidate genes. These identified signals can be characterized by using young and elderly genetically modified mice after LT training. Our search for genes modulated in the skeletal muscle by LT training, which can be easily and safely performed by elderly individuals, highlighted the importance of mitochondrial function and extracellular matrix (ECM) remodeling in the skeletal muscle after LT training. A novel candidate gene, Secreted Protein Acidic and Rich in Cysteine (SPARC), controls ECM remodeling and plays an important role in skeletal muscle maturation. SPARC is also known to interact with a protein which induces mitochondrial biogenesis. Therefore, we hypothesized that SPARC is mediating enhanced muscle integrity promoted by mild-exercise training in elderly individuals, thus contributing to the improvement of sarcopenia and related metabolic diseases.
\end{abstract}

The first objective is to demonstrate the roles of SPARC in the link between ECM remodeling and mitochondrial function and OXPHOS capacity in mouse myoblasts. The gene and protein expressions of markers for differentiation, ECM remodeling, mitochondrial biogenesis and OXPHOS capacity, as well as antioxidant enzyme activities, mitochondrial membrane potential, reactive oxygen species production and damage will be analyzed. The second objective is to elucidate the effects of SPARC on exercise-induced changes in skeletal muscle, such as cell growth, ECM modification, and mitochondrial biogenesis and increased OXPHOS capacity, by using electronic pulse stimulation/mechanical stretch models in mouse myoblasts. In addition to the measurements of the first objective, we are studying the cell proliferation, phosphorylations of proteins in the insulin- 
stimulated protein synthesis pathway, and glucose uptake. The last objective is to characterize the functional relevance of SPARC in sarcopenia and skeletal muscle adaptations to mild exercise in Sparc KO mice. Blood, liver, abdominal adipose tissues and skeletal muscle are sampled for the measurements of glucose and lipid metabolism as well as mitochondrial biogenesis and OXPHOS. Markers for ECM remodeling, proliferation and differentiation are also quantified in the skeletal muscle. Other groups of mice, from 10 weeks and 16.5 months of age, are subjected to exercise for 6 weeks. This project will provide the functional characterization of SPARC controlling the beneficial effects of physical exercise against obesity and aging. Consequently, it may impact the development of therapy strategies for sarcopenia, obesity, diabetes and coronary artery diseases. A long-term possibility is to develop treatments for obesity and related diseases in human with an exercise pill.

\title{
Mex3c Mutation in Mouse Reduces Adiposity Partially Through Increasing Physical Activity
}

\author{
Baisong Lu*, Changjie Han, Yan Jiao and Qingguo Zhao \\ Institute for Regenerative Medicine, Wake Forest University Health Sciences, Winston-Salem, NC, USA
}

\begin{abstract}
Mex3c is an RNA-binding protein with unknown physiological function. We recently reported that a Mex3c mutation in mice causes growth retardation and reduced adiposity, but how adiposity is reduced remains unclear. Here we show that homozygous Mex3c gene trap mice have increased physical activity. The Mex3c mutation consistently conferred full protection from diet-induced obesity, hyperglycemia, insulin resistance, hyperlipidemia, and hepatic steatosis. In ob/ob mice with leptin deficiency, the Mex3c mutation also increased physical activity and improved glucose and lipid profiles. Expressing cre in neurons of Mex3c gene trap mice, an attempt to partially restoring neuronal Mex3c expression, significantly increased white adipose tissue deposition, but had no effects on body length. Our data suggest that one way in which Mex $3 \mathrm{c}$ regulates adiposity is through controlling physical activity, and that neuronal Mex3c expression could play an important role in this process.
\end{abstract}

\section{Skeletal Muscle Metabolites Potentially Involved in Favorable Energy Metabolism in Anti-Obesity Lipoprotein Lipase Transgenic Rabbits}

\author{
Yuichiro Nishida ${ }^{1 *}$, Keitaro Tanaka ${ }^{1}$, Kazutoshi Nishijima ${ }^{2}$, Fumika Mi-ichi ${ }^{3}$, Yosuke Yamada ${ }^{4}$ Hiroaki Tanaka $^{5}$, Jianglin \\ $\operatorname{Fan}^{6}$ and Shuji Kitajima ${ }^{7}$ \\ ${ }^{1}$ Department of Preventive Medicine, Faculty of Medicine, Saga University, Saga, Japan \\ ${ }^{2}$ Animal Research Laboratory, Bioscience Education-Research Center, Akita University, Akita, Japan \\ ${ }^{3}$ Department of Biomolecular Sciences, Faculty of Medicine, Saga University, Saga, Japan \\ ${ }^{4}$ Department of Nutritional Science, National Institute of Health and Nutrition, Tokyo, Japan \\ ${ }^{5}$ Laboratory of Exercise Physiology, Faculty of Health and Sports Science, Fukuoka University, Fukuoka, Japan \\ ${ }^{6}$ Department of Molecular Pathology, Interdisciplinary Graduate School of Medicine and Engineering, University of Yamanashi, \\ Yamanashi, Japan \\ ${ }^{7}$ Division of Biological Resources and Development, Analytical Research Center for Experimental Sciences, Saga University, Saga, Japan
}

\begin{abstract}
Lipoprotein lipase (LPL) transgenic ( $\mathrm{Tg}$ ) rabbits have been reported to be protected against high-fat-diet (HFD)-induced obesity. Skeletal muscle is a major organ responsible for energy metabolism. We analyzed muscle metabolites profile, and simultaneously measured whole body energy expenditure (EE) in LPL Tg and control (wild type) rabbits under HFD condition. At the $15^{\text {th }}$ week of 16 -week HFD period, whole body EE was measured using doubly-labeled water $\left(2 \mathrm{H}_{2} \mathrm{O}\right.$ and $\left.\mathrm{H}_{2}{ }^{18} \mathrm{O}\right)$ method in the $\mathrm{Tg}(\mathrm{n}=12)$ and wild-type (control, $\mathrm{n}=12)$ rabbits. Gastrocnemius muscle (red portion) of HFD-fed rabbits $(\mathrm{n}$ = 9, each group) were used for metabolomic profiling using capillary electrophoresis mass spectrometry. 165 peaks (98 in cation mode and 67 in anion mode) were detected. Body weight was significantly lower in $\mathrm{Tg}$ than controls, while EE levels were significantly higher in Tg compared with controls $(94.0 \pm 6.5$ vs. $88.1 \pm 7.1 \mathrm{kcal} / \mathrm{kg}$ weight $/$ day, $\mathrm{P}<0.05)$. Among $165 \mathrm{muscle}$ metabolites measured, concentration levels of 40 metabolites were significantly different between the two groups. To pare down the most promising candidate metabolites, we further performed the correlation analyses to identify muscle metabolites that are significantly correlated with the whole body EE among the 40 metabolites. As a result, we found 18 muscle metabolites that were positively or inversely correlated with the whole body EE. For instance, higher concentration levels of branched-chain amino acids (isoleucine, leucine) and other amino acids (methionine, asparagine) were significantly associated with a higher level of EE, while higher $\beta$-alanine concentrations were associated with lower EE levels. Two metabolites related to carnitine
\end{abstract}


(octanoylcarnitine and butyrylcarnitine) were also inversely correlated with the EE. Higher level of glycerol concentration was associated with an enhanced level of EE, whereas higher NADH level was associated with a decreased level of EE. Thus, the current study identified the skeletal muscle metabolites and metabolic pathways that may be involved in the favorable regulation of energy metabolism in the anti-obesity LPL Tg rabbits.

\title{
Genetics of Food Addiction
}

\author{
Marilyn C. Cornelis ${ }^{*}$, Alan Flint ${ }^{2}$, Alison E. Field ${ }^{2}$, Eric B. Rimm ${ }^{2}$, Peter Kraft ${ }^{2}$, Jiali Han ${ }^{3}$ and Rob M. van Dam ${ }^{4}$ \\ ${ }^{1}$ Preventive Medicine, Northwestern University Feinberg School of Medicine, Chicago, IL, USA \\ ${ }^{2}$ Harvard School of Public Health, Boston, MA, USA \\ ${ }^{3}$ Richard M. Fairbanks School of Public Health, Simon Cancer Center, Indiana University, Indianapolis, IN, USA \\ ${ }^{4}$ Saw Swee Hock School of Public Health and Department of Medicine, Yong Loo Lin School of Medicine, National University of \\ Singapore and National University Health System, Singapore
}

\begin{abstract}
Evidence of parallels between drug addiction and eating behavior continues to accumulate. Genetic studies of addictive substances have yielded a number of susceptibly loci that point to common higher-order genetic pathways underlying addiction. To elucidate the relationship between drug addiction and eating behaviors we conducted a genome-wide association study (GWAS) of food addiction, determined by the modified Yale Food Addiction Scale (mYFAS), among 9,314 U.S. women of European ancestry and examined results for enrichment of single-nucleotide polymorphisms $(\mathrm{SNPs})(\mathrm{n}=44)$, genes $(\mathrm{n}=238)$ and pathways $(\mathrm{n}=11)$ implicated in drug addiction. Two loci met $\mathrm{GW}$-significance $\left(\mathrm{P}<2.5 \times 10^{-8}\right)$ mapping to $17 \mathrm{q} 21.31$ and 11q13.4 that harbor genes with no obvious roles in eating behavior. GW results were significantly enriched for gene members of the MAPK signaling pathway $(\mathrm{P}=0.02)$. No candidate SNP or gene for drug addiction was significantly associated with food addiction. A SNP previously linked to illicit drug use, and the candidate genes LOC100130673, HOMER1, ZHX2, DRD2, and SURF6, were nominally associated with food addiction. A global exploratory pathway analysis yielded significant enrichment for interleukin signaling genes (FDR = 0.003). In the first GWAS of mYFAS, we identified suggestive loci worthy of further follow-up but provide limited support for shared genetic underpinnings of food addiction and drug addiction.
\end{abstract}

\section{Potential Discovery of Novel High Fat Diet Induced Satiety Hormone}

\author{
St-Amand Jonny and Yoshioka $M$ \\ Functional Genomics Laboratory, Department of Molecular Medicine, University Laval, Canada
}

\begin{abstract}
With the new era, the word wide epidemic of obesity brings obesity as the most critical risk factors for complex diseases. High fat (HF) diet is a well-known and obvious cause of obesity. The satiety hormone for glucose, the insulin, is well known and characterized. Since the lipid metabolism is the absolute main component of energy equilibrium, we can expect that the evolution of mammalians will have also equip the human body with a hormone which will be the specific sensor for HF intake and will inform the hypothalamus to give the order to stop to eat, since the energy equilibrium is finely tuned to keep a stable body weight set point along the decencies. However, no such HF diet induced satiety hormone has been yet characterized. According to the current obesity epidemics and its associations with complex diseases, the studies focused to discover the $\mathrm{HF}$ diet induced satiety hormone are thus urgently needed. Our strategy is to discover the HF diet induced satiety hormone usually transcriptionally regulated $30 \mathrm{~min}$ to $3 \mathrm{~h}$ after $\mathrm{HF}$ meal and to deliver it at the time of appetizer in order to control HF intake, obesity and related complex diseases. We have characterized, for the first time, the transcriptomes of several tissues involved in energy intake and expenditure as well as their regulation by factors which influence energy homeostasis and body fat distribution. Since the acute changes in the expression levels of all the genes induced by HF intake had not been previously studied in key tissues regulating appetite and satiety, the initial events in the dysregulation of appetite and satiety, which are responsible for the development of obesity, remained unclear. We have investigated the effects of HF or LF meal on the transcriptomes of stomach (absorption of short fatty acids), intestine (digestion and absorption of lipids), adipose tissue (storage of lipids and hormone secretions) and hypothalamus (the brain center controlling food intake), pituitary (important gland for hormone secretions) in order to identify the HF-specific signals. We have identified hundreds of genes modulated after HF or low fat $(\mathrm{LF})$ meal ingestion. In order to further characterize the roles of the key genes in feeding behavior and development of obesity, we have made a selection of the novel candidate genes.
\end{abstract}

Trefoil factor family member 2 (TFF2) is a small gut peptide, mainly known for its protective and healing functions. As 
previously demonstrated, high-fat (HF) feeding can rapidly and specifically modulate Tff2 transcription in key tissues of mice, including the duodenum and mesenteric adipose tissue, therefore suggesting a novel role for this gene in energy balance. To explore whether and how Tff2 can influence feeding behavior and energy metabolism, we have challenged Tff 2 knock-out (KO) mice with HF diet for 12 weeks. Tff2 KO mice showed a greater appetite and higher energy intake compared to wildtype (WT) mice. Consistently, they presented lower levels of serum leptin, and increased transcription of agouti-related protein (Agrp) in the hypothalamus. Though energy and triglyceride fecal excretion were augmented in Tff2 KO mice, digestible energy intake was superior. However, $\mathrm{KO}$ mice were finally protected from HF diet-induced obesity, and accumulated less weight and fat depots than WT animals, while keeping a normal lean mass. Energy efficiency was lower in HF-KO mice, while energy expenditure and locomotor activity were globally increased. Our work demonstrates previously unsuspected roles for Tff 2 and suggests it to be a mastermind in the control of energy balance and a promising therapeutic target for obesity. Overall, this project will elucidate the mechanisms underlying the anti-obesity effect of Tff2 inhibition, and will contribute to the understanding of energy balance control. A long-term possibility is to develop treatments for obesity and related diseases in human. This study characterizes a potential therapeutic target which can be also used for the treatment of obesity, diabetes and CAD by administration of the pharmaceutical inhibitors.

\title{
Synoviolin, as a Novel Factor for Understanding Obesity and Metabolic Disorders Based on Chronic Inflammation
}

\author{
Toshihiro Nakajima \\ Institute of Medical Science, Tokyo Medical University, Japan
}

\begin{abstract}
Inflammation by itself is classical pathogenic concept but still very crucial for understanding many disorders even in 21 st century. In ancient Roman periods, Celsus and Galenus described that the classical signs of acute inflammation are pain, heat, redness, swelling and loss of function. The purpose of inflammation is to eliminate the initial cause of cell injury, clear out necrotic cells and tissues damaged from the original insult and the inflammatory process, and to initiate tissue repair. By contrast, chronic inflammation is recently thought to lead to a host of intractable diseases, including rheumatoid arthritis, atherosclerosis, cancer, diabetes mellitus, and obesity. My research team discovered Synoviolin (SYVN1) as an over expressing molecule in rheumatoid synovium (Amano et al., 2003), and has been verifying its critical roles in arthritis, inflammatory cytokine-signaling and fibrosis (Yagishita et al., 2005, 2008, 2014). Recently, a gene knockout strategy was used in post-neonatal mice to delete Syvn1 and its deficiency resulted in weight loss and lower accumulation of white adipose tissue in otherwise wild-type animals as well as in genetically obese (ob/ob and $\mathrm{db} / \mathrm{db}$ ) and adipose tissue-specific knockout mice as compared to control animals. SYVN1 interacted with and ubiquitinated the thermogenic co activator peroxisome proliferators activated receptor co-activator (PGC)$1 \alpha$ and Syvn1 mutants showed up regulation of PGC- $1 \alpha$ target genes and increase in mitochondrion number, respiration, and basal energy expenditure in adipose tissue relative to control animals (Fujita et al., 2016). Moreover, the selective SYVN1 inhibitor LS-102 abolished the negative regulation of PGC-1 $\alpha$ by SYVN1 and prevented weight gain in mice. Thus, SYVN1 is a novel regulator of chronic inflammation and a potential therapeutic target in treatment with those diseases. This study will also demonstrate the data on newly developed herbal medicine in Japanese market.
\end{abstract}

\section{Characterization of Retinal Energy Metabolism in Age-related Macular Degeneration}

\author{
Jean-Sebastien Joyal \\ Université de Montreal, Canada
}

\begin{abstract}
Tissues with high metabolic rates often use lipid as well as glucose for energy, conferring a survival advantage during feast and famine. Current dogma suggests that high-energy consuming photoreceptors depend on glucose. We recently showed that retina also uses fatty acid (FA) $\beta$-oxidation for energy. In a murine model of age-related macular degeneration (AMD), dysregulated lipid and glucose photoreceptor energy metabolism was a driving force in neovascular AMD. Using examples from our recent work, this presentation will describe old and new approaches to characterize the energy metabolism of mice retinas.
\end{abstract}




\title{
Personalized Prevention and Intervention for Obesity: Implications of Gene- Environment Interaction
}

\author{
Qibin Qi \\ Department of Epidemiology Eo Population Health, Albert Einstein College of Medicine, Bronx, NY 10461, USA
}

\begin{abstract}
Obesity and its co-morbidity have become major public health problems throughout the world. It has been acknowledged that obesity is a result of interplay between genetic and environment factors, though the underlying mechanisms remain unclear. A large body evidence has shown that sedentary lifestyle and high-density energy food intake are major risk factors for overweight and obesity. On the other hand, with the advent of genome-wide association studies, many genetic loci have been identified to be associated with obesity and related traits. Our prior work has identified significant interaction between obesogenic diet/lifestyle (e.g., high sugar-sweetened beverage and fried food consumption, low physical activity) and genetic variants on BMI and obesity risk in large population-based cohorts. In addition, significant inter-individual variation in weight loss in response to diet/lifestyle interventions has long been noted, partly accounted for by an individual's genetic make-up. Thus, a better understanding of interactions between genetic and environment factors may help to develop more effective strategies for obesity prevention and intervention. In this review, we will summarize recent findings of gene-die/lifestyle interactions on obesity in observational studies as well as genetic influence on weight loss in response to diet/lifestyle interventions, and discuss the potential applications of these findings for the "personalized" obesity prevention and intervention.
\end{abstract}

\section{Featured Presentations II}

\section{Determinants of Immune-muscle Crosstalk in Obesity and Diabetes}

\author{
M. Constantine Samaan ${ }^{1,2}$ \\ ${ }^{1}$ Division of Pediatric Endocrinology, McMaster Children's Hospital, Canada \\ ${ }^{2}$ Department of Pediatrics, McMaster Children's Hospital, Canada
}

\begin{abstract}
Obesity and type 2 diabetes are global epidemics that together affect billions of people around the world. Over the past few years, broad understanding of the mechanisms driving obesity and diabetes emerged. One key mechanism that links these two diseases involve the activation of the immune system in obesity its association with inflammation, which can drive insulin resistance and type 2 diabetes. While this has been extensively described in adipose tissue, limited data exist to associate muscle immune cells with diabetes. Skeletal muscle is the key organ responsible for postprandial glucose disposal, and its insulin resistance is an early event in obesity; this may represent a potential therapeutic target. In this presentation, data from our research group will be presented to highlight muscle immune responses in obesity. In addition, data on novel interventions that can alter muscle-immune crosstalk will be discussed.
\end{abstract}

\section{Influence of Testosterone Replacement Therapy on Metabolic Disorders in Patient with Type 2 Diabetes Mellitus and Androgen Deficiency}

\author{
Shota Janjgava ${ }^{1,2^{*}}$, Tamr Zerekidze ${ }^{1,2}$, Lasha Uchava ${ }^{1,2}$, Elene Giorgadze ${ }^{1,2}$ and Ketavan Asatiani ${ }^{1}$ \\ ${ }^{1}$ National Institute of Endocrinology, Tbilisi, Georgia \\ ${ }^{2}$ Tbilisi State University, Tbilisi, Georgia
}

\begin{abstract}
Introduction: Over the past few decades, obesity and diabetes mellitus (T2D) has become a global health challenge. Between 1980 and 2004 the prevalence of obesity increased from 15 to 33\% in the United States - a pattern mirrored across the world. Multiple epidemiological studies have shown that low testosterone levels are associated with and predict the future development of T2D and the metabolic syndrome.
\end{abstract}

Aim of Study: The aim of study was to show the influence of testosterone replacement therapy on obesity, HbA1c level, 
arterial hypertension and dyslipidemia with patient diabetes mellitus and Androgen deficiency.

Materials and Methods: 125 male patients with diabetes mellitus was screened, 85 subjects with 41-65 years and BMI $27.0-48.0 \mathrm{~kg} / \mathrm{m}^{2}$ were randomized in placebo-controlled study, who underwent a routine physical examination and choose free testosterone examination. According to the laboratory and clinical condition we divided patients into two groups: First group, treatment group and Second group, placebo group. In the first group we used diet, physical activity [Lifestyle intervention implies reduced calorie diet; the reduction of daily calorie intake in 800-1200 calorie, it was selected individually], patient's antidiabetic therapy and testosterone replacement therapy (TRT), [testosterone undecanoate $250 \mathrm{mg} / \mathrm{ml}$ intra- muscular $3 \mathrm{months,} 1$ time]. In second group we used diet, physical activity [Lifestyle intervention implies reduced calorie diet; the reduction of daily calorie intake in 800-1200 calorie, it was selected individually], patient's antidiabetic therapy and placebo.

Results: After six months of treatment we repeated the diagnostic assessments. We had some positive results cholesterol, triglyceride and LDL levels decreased, and HDL increased both of group but better results was in first group which was clinically significant. Free testosterone level increased in all groups but the best results was in first group which was clinically significant where was used of testosterone undecanoate. HbA1c decreased in both group but in first group we had the best result. BMI decreased in both groups but more reduction was in first group. Leptin level after treatment was approximately same in both groups, but compared best results were achieved in first group, also blood pressure were reduced in both group, where we found alike results.

Discussion: Serum testosterone, glycosylated hemoglobin, high-density lipoprotein cholesterol, triglyceride concentrations, BMI and hypertension improved in both treatment groups after 26 weeks of treatment. We have shown that testosterone replacement therapy improves insulin resistance and glycemic control in hypogonadal men with diabetes.

Conclusion: Our study demonstrated that it is possible to break into this vicious circle by raising testosterone levels in diabetic men and low testosterone level. In addition to traditional CV risk factors, novel risk factors are also inversely related to testosterone levels. Re-instituting physiological levels of testosterone in hypoandrogenic men as our small study shown, have an important role in reducing the prevalence of diabetic complication.

\title{
Effects of Diet Quality, Obesity, and Inflammation on Mortality from Cancer and Other Chronic Diseases
}

\section{Marisa A. Bittoni", Chris Taylor, Colleen Spees and Steven Clinton}

The Obio State University, Columbus, OH, USA

\begin{abstract}
Introduction: Diet quality, obesity and other lifestyle factors have shown consistent associations with chronic disease mortality. The present study simultaneously assessed the effects of diet quality, obesity and inflammation on cancer/chronic disease mortality. We hypothesized that reduced diet quality, increased obesity and elevated inflammation would increase mortality risk from cancer and other chronic diseases.
\end{abstract}

Methods: Data from The National Health and Nutrition Examination Survey (NHANES) III participants were used to prospectively examine the effects of diet quality (measured by the Healthy Eating Index), obesity (BMI) and inflammation (C-reactive protein levels) on mortality risk from cancer, all causes, cardiovascular disease (CVD) and diabetes. Cox proportional hazards regression was performed to assess these relationships.

Results: The regression results revealed that higher HEI scores showed significantly reduced risks of mortality for cancer (11\%), all causes (7\%) and CVD (5\%) when adjusted for other lifestyle factors. Elevated CRP was associated with a 30\% increased risk of mortality from cancer, all cause and CVD, and a 2-fold increased risk of diabetes mortality. Increased obesity showed slightly reduced mortality for all but diabetes ( $5 \%$ increased risk).

Discussion: The results support our hypothesis that healthier eating is associated with decreased cancer/chronic disease mortality after adjusting for other lifestyle factors. The results also indicate that higher inflammation levels were independently associated with cancer/chronic disease mortality. Obesity, however, was not a prominent factor when adjusted for healthy eating and inflammation. Future studies should continue to assess these factors and their inter-relationships so that recommendations can be made to reduce mortality for cancer and other chronic diseases. 


\title{
Leptin/Adiponectin Ratio as a Predictor of Postprandial Triglyceride Clearance in Obese Subjects
}

\author{
Larsen MA*, Isaksen V, Goll R, Lekahl S, Moen OS and Florholmen J \\ Research Group of Gastroenterology and Nutrition, Institute of Clinical Medicine, Norwegian Arctic University, Tromso, Norway
}

\begin{abstract}
Background: Obesity is a global health issue and can cause serious diseases including cardiovascular disease and diabetes.

Aim: To study the relationship between insulin resistance, leptin and adiponectin in response to postprandial triglyceridemia.

Material \& Methods: A total of 27 subjects with obesity (BMI > 30); 21 metabolic healthy obese (MHO), and 6 with metabolic disorders volunteered to participate in this study. In addition, 16 age- and sex-matched, healthy and normal weight subjects represented the control group. Postprandial triglycerides (TG) were measured in serum in an oral fat tolerance test (OFTT) during $8 \mathrm{~h}$, and TG response (TGR) was calculated. Insulin sensitivity (HOMA-IR and WBISI), the atherogenic index; leptin: adiponectin ratio (L: A ratio) and leptin sensitivity by the indirect basal metabolic rate (BMR)/serum leptin index was measured. The control subjects performed the same tests.
\end{abstract}

Results: A significant interaction between L: A ratio versus SE-TG at various time points and time course was detected in each group. Fasting TG could predict IR defined as L: A ratio $>1.2$, with a cut-off value of TG $>1.08 \mathrm{mmol} / \mathrm{L}$, and leptin resistance (defined as $>114.5$ ), with a cut off value of TG $>1.3 \mathrm{mmol} / \mathrm{L}$. Having L: A ratio $>1.2$ was associated to a 30 times more likeliness of having a pathological TGR.

Conclusion: In obese subjects, also including subjects with no clinical signs of metabolic syndrome there are early disturbances with delayed clearance of postprandial TG, and L: A ratio may be a useful clinical surrogate marker of delayed postprandial TG clearance.

\section{Metabolism of Anticancer Agents Noscapine and Analogs}

\author{
Ramesh Chandra* and Vartika Tomar \\ Laboratory of Drug Discovery and Metabolism, Department of Chemistry, University of Delhi, Delhi, India
}

\begin{abstract}
Many nitrogen-moiety containing alkaloids derived from plant origins are bioactive and play a significant role in human health and emerging medicine. Noscapine is an isoqiunoline alkaloid found in opium latex. Unlike most other alkaloids obtained from opium latex, noscapine is not sedative and has been used as antitussive drug in various countries. Recently, it has been introduced as an anti-mitotic agent. This drug can be used orally. When the resistance to other anti-cancer drugs such as paclitaxel menifests, noscapine might be effective. Therefore, noscapine and its analogs have great potential as novel anticancer agents. From the urine of rats, rabbits and humans treated with noscapine, two novel metabolites were isolated and identified as 7-hydroxy-6-methoxyphthalide (MA-1) and 6-hydroxy-7-methoxyphthalide (MA-2), mainly by mass spectrometry.

Our laboratory has reported that two brominated derivatives of noscapine, 5-bromonoscapine (5-Br-nosc) and reduced 5-bromonoscapine ( $\mathrm{Rd} 5$-Br-nosc), have higher tubulin binding activity than noscapine and affect tubulin polymerization differently from noscapine. In addition, they are able to arrest cell cycle progression at mitosis at concentrations much lower than noscapine. Interestingly, whereas noscapine-arrested cells have nearly normal bipolar spindles, cells arrested by 5-Brnosc and $\mathrm{Rd} 5-\mathrm{Br}$-nosc form multipolar spindles. Nevertheless, noscapine and the two derivatives all affect the attachment of chromosomes to spindle microtubules and they impair the tension across paired kinetochores to similar degrees. 5- Br-nosc and $\mathrm{Rd} 5-\mathrm{Br}$-nosc are also more active than noscapine in inhibiting the proliferation of various human cancer cells, including those that are resistant to paclitaxel and epothilone. Our lab investigation thus indicates a great potential for the use of 5-Br-nosc and $\mathrm{Rd} 5-\mathrm{Br}$-nosc both as biological tools for studying microtubule-mediated processes and as chemotherapeutic agents for the treatment of human cancers.

We have synthesized various noscapine derivatives to describe recent breakthroughs that have led to an elucidation of the noscapine biosynthetic pathway, and to discuss the pharmacological properties that have prompted intensive evaluation of the potential pharmaceutical applications of noscapine and several semi-synthetic derivatives. We have speculated the future potential for the production of noscapine using metabolic engineering and synthetic biology in plants and microbes.
\end{abstract}

Noscapine, a phthalideisoquinoline alkaloid derived from Papaver somniferum, has been used as a cough suppressant since the mid1950s, illustrating a good safety profile. Noscapine has since been discovered to arrest cells at mitosis, albeit with 
moderately weak activity. Immunofluorescence staining of microtubules after $24 \mathrm{~h}$ of noscapine exposure at $20 \mu \mathrm{M}$ elucidated chromosomal abnormalities and the inability of chromosomes to complete congression to the equatorial plane for proper mitotic separation. A number of noscapine analogues possessing various modifications have been described within the literature and have shown significantly improved antiprolific profiles for a large variety of cancer cell lines. Several semisynthetic antimitotic alkaloids are emerging as possible candidates as novel anticancer therapies.

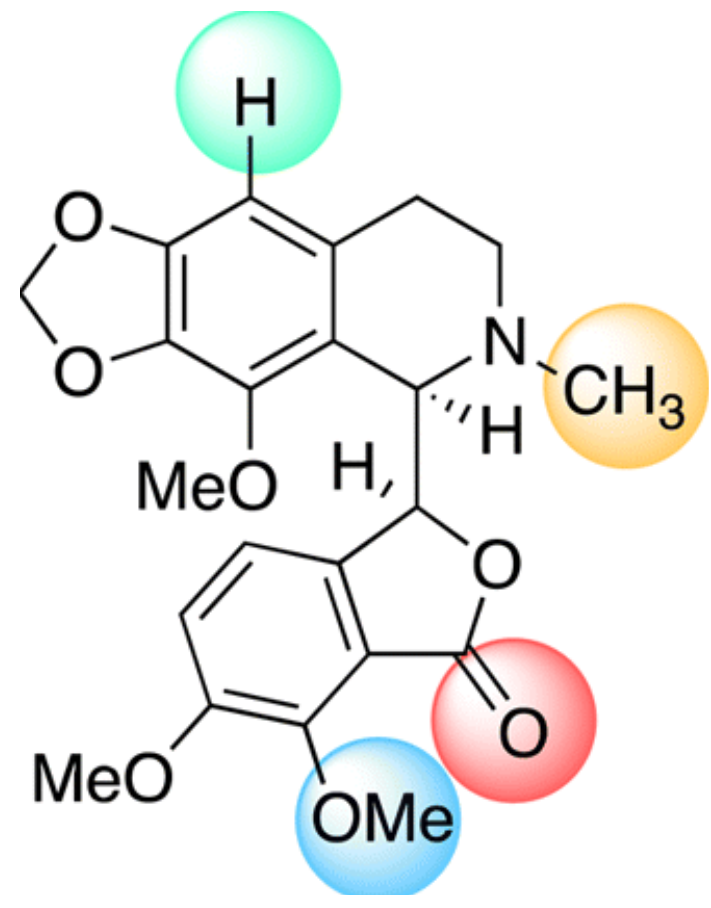

\title{
Achievement of MEtabolic syndRome and abdominaL ObesiTy (MERLOT) Study and Development of Dual BIA Method for Visceral Adiposity
}

\author{
Kazuwa Nakao ${ }^{*}$, Yoko M. Nakao ${ }^{2}$, Kazuhiro Nakao ${ }^{2}$, Yoshihiro Miyamoto², Kenji Kangawa ${ }^{2}$, Takashi Miyawaki ${ }^{3}$, Shinji \\ Yasuno $^{4}$, Kenji Ueshima ${ }^{4}$, Nobuo Saito ${ }^{5}$ and Toshikazu Shiga ${ }^{6}$ \\ ${ }^{1}$ Medical Innovation Center, Kyoto University, School of Medicine, Kyoto, Japan \\ ${ }^{2}$ National Cerebral and Cardiovascular Center, Suita, Japan \\ ${ }^{3}$ Kyoto Women's University, Kyoto, Japan \\ ${ }^{4}$ Institute for Advancement of Clinical and Translational Science, Kyoto University Hospital, Kyoto, Japan \\ ${ }^{5}$ NTT West Kyoto Hospital, Kyoto, Japan \\ ${ }^{6}$ Omron HealthCare Co., Kyoto, Japan
}

\begin{abstract}
MERLOT study is the longitudinal cohort study to investigate the significance of intra-abdominal fat area (IAFA) as a predictor on new onset of the metabolic syndrome (MetS). MERLOT Study 1: Of 25,255, 1,380 measured IAFA by computer tomography and had no metabolic syndrome components (MetS-c). During 3.6 years of the mean follow-up, 752 occurred one of MetS-c. IAFA was significantly associated with new onset of MetS-c (adjusted HR: 1.05/10 cm², 95\%CI: 1.03-1.07), independent of BMI, and even in non-obese with BMI $<25 \mathrm{~kg} / \mathrm{m}^{2}$. MERLOT study 2: Of 2,743 subjects without two or more of MetS-c (clustering), 797 of clustering occurred. Classifying participants according to IAFA and BMI, the highest risk was observed among those who were IAFA $\geq 100 \mathrm{~cm}^{2}$ and BMI $\geq 25 \mathrm{~kg} / \mathrm{m}^{2}(\mathrm{p}<0.001)$. Higher IAFA was associated with an increased risk for clustering within each BMI category $(\mathrm{p}<0.001)$. MERLOT study demonstrates that increased IAFA is an independent predictor for new onset of MetS-c and clustering among non-obese and obese. IAFA and BMI assessed in combination can improve prediction of clustering of MetS-c. Dual BIA method: We succeeded in developing the novel compact equipment with the dual bio-impedance (dual BIA method) to measure IAFA without radiation exposure. IAFA measured with the dual BIA method is highly correlated with IAFA measured with CT $(r=0.821, p<0.001)$ Taken together, MERLOT study demonstrates that IAFA is an important predictor of MetS and the dual BIA which enables to measure IAFA without radiation exposure is a useful method for diagnosis and follow-up of MetS and obesity.
\end{abstract}




\title{
Physiological Property of Luteolin in Diet-induced Obese Mice
}

\author{
Myung-Sook Choi ${ }^{1,2^{*}}$ and Eun-Young Kwonand ${ }^{1,2}$ \\ ${ }^{1}$ Center for Food and Nutritional Genomics Research, South Korea \\ ${ }^{2}$ Department of Food Science and Nutrition, Kyungpook National University, South Korea
}

\begin{abstract}
Little is known about the mechanism associated with the effect of luteolin on inflammation-related obesity. The aim of the present study was to reveal the effect of luteolin on anti-inflammation in diet-induced obesity by integrating transcriptional responses with phenotype biomarkers. C57BL/6J mice were fed a normal diet (ND), a high-fat diet (HFD, 20\% fat), and a HFD containing $0.005 \%$ (w/w) luteolin for 16 weeks. Body weight, all white adipose tissue (WAT) depot weights and adipocyte size were significantly decreased by luteolin supplementation compared to HFD. Luteolin was effective for lowering the plasma and hepatic lipid levels in diet-induced obese mice by altering the hepatic lipid metabolizing enzyme activities and fecal lipids contents. Furthermore, luteolin was beneficial against HFD-induced inflammation by decreasing plasma cytokine level and pro-inflammatory protein expression. IPA revealed upstream regulator on significant networks targeted by luteolin was consisted of genes related to lipid synthesis and oxidation (PPARA and PPARD) in liver and inflammatory response (LEP, INSR and IL6) in epididymal adipose tissue. In liver, luteolin down-regulated gene expression associated with lipid and cholesterol synthesis, while it up-regulated gene expressions associated with fatty acid oxidation. In adipose tissue, luteolin up-regulated gene expressions related to fatty acid oxidation and TCA cycle, and it down-regulated gene expression of ADRP which involves in formation of lipid droplet. Results indicate that luteolin supplement exhibits positive effects on metabolic responses in obesity by modulating the metabolites associated with inflammation. Anti-obesity effect of luteolin could help to ameliorate the deleterious effects of HFD-induced inflammation.
\end{abstract}

\section{Childhood Health Status and Adulthood Cardiovascular Disease Morbidity in China: Are They Related?}

\author{
Qing Wang \\ School of Business, Dalian University of Technology, Dalian, Liaoning, China
}

\section{Abstract}

Background: Cardiovascular diseases are among the top health problems of the Chinese people. Although mounting evidence suggests that early childhood life circumstances have an enduring effect on late life chronic morbidity, none study so far has analyzed the issue in China. This study aimed to examine long-term effects of childhood health status on adulthood cardiovascular disease in China.

Methods: Using nationally representative data from China Health and Retirement Longitudinal Study 2013 (CHARLS), Logit model and difference-in-differences estimator were applied to analyze the relationship between childhood health status and adulthood cardiovascular disease.

Results: Good childhood health status decreased the likelihood of self-reported adult cardiovascular diseases by 1.8 percent $(p<0.01)$. The marginal effects of child health status on adult cardiovascular diseases was 1.4 percent $(p<0.05)$ for the females, while 2.2 percent $(\mathrm{p}<0.01)$ for the males.

Conclusion: A negative relationship exists between good childhood health and adulthood risk for cardiovascular diseases. Good childhood health decreases morbidity of cardiovascular diseases in one's adult life. Given the long-term effect of childhood health on the adulthood health later on, health policy and programs to improve health status and well-being of Chinese populations over the entire life cycle, especially in persons' early life, are expected to be effective and successful.

\section{Vitamin D Deficiency is a Prevalent Condition in Obesity: Increase in Body Fat Mass Leads to a Deterioration of Vitamin D Deficiency}

\section{Edita Stokic}

Department of Endocrinology, Diabetes and Metabolic Diseases, Clinical Centre of Vojvodina, Novi Sad, Serbia 


\title{
Abstract
}

Vitamin D has an important role for the regulation of calcium homeostasis, and is suggested to supply a protective effect against multiple diseases and conditions. Serum concentrations of 25-hydroxyvitamin D, $25(\mathrm{OH}) \mathrm{D}$, are considered as the best indicator of total body vitamin $\mathrm{D}$ stores, and its value reflects the total dietary intake and exposure to ultraviolet radiation. Numerous clinical and epidemiological studies have proposed a relationship between vitamin D level, obesity and insulin resistance. Additionally, in obese patients, there is a suggestion that insulin resistance improves after vitamin D supplementation.

The study was carried out in a group of 50 obese patients (BMI $43.5+9.2 \mathrm{~kg} / \mathrm{m}^{2}$ ) and 36 normal weight participants (BMI: $22.6+1.9 \mathrm{~kg} / \mathrm{m}^{2}$ ). We excluded patients with recent weight changes and those who had been treated with vitamin D within 3 months prior. The prevalence of vitamin D deficiency $(25(\mathrm{OH}) \mathrm{D}<50 \mathrm{nmol} / \mathrm{L})$ was $88 \%$ among obese patients and $31 \%$ among non-obese individuals. The mean serum $25(\mathrm{OH}) \mathrm{D}$ level was significantly lower in the obese group than in the control group $(27.3+13.7$ vs. $64.6+21.3 \mathrm{nmol} / \mathrm{L} ; \mathrm{P}<.001)$. The coefficients of linear correlation between $25(\mathrm{OH}) \mathrm{D}$ levels and anthropometric parameters (body weight, BMI, fat \%) are negative and significant $(\mathrm{P}<.001)$. The increase in $\mathrm{BMI}$ and $\mathrm{BF} \%$ was associated with a downward trend of $25(\mathrm{OH}) \mathrm{D}$ concentrations. Furthermore, increase in body fat mass leads to a deterioration of vitamin D deficiency.

Our results have demonstrated an association between changes in BMI, total fat mass and $25(\mathrm{OH}) \mathrm{D}$ level. The increase in $\mathrm{BMI}$ and $\mathrm{BF} \%$ was associated with a downward trend of $25(\mathrm{OH}) \mathrm{D}$ concentrations. Measurement of vitamin D levels may help to identify obese individuals at greater risk and this could be a basis for the design of intervention trials in the obese patients.

\section{Influence of Obesity on the Correlation Between Laryngopharyngeal Reflux and Obstructive Sleep Apnea}

\author{
Marcos Marques Rodrigues ${ }^{1 *}$, Ralph Silveira Dibbern ${ }^{2}$, Victor Jose Barbosa Santos ${ }^{3}$ and Luis Augusto Passeri ${ }^{4,5}$ \\ ${ }^{1}$ School of Medicine, Centro Universitario de Araraquara (UNIARA), Araraquara, SP, Brazil \\ ${ }^{2}$ School of Medicine, Universidade de Sao Paulo (USP), Ribeirao Preto, SP, Brazil \\ ${ }^{3}$ School of Medicine, Universidade EstadualPaulista (UNESP), Botucatu, SP, Brazil \\ ${ }^{4}$ Southwestern Medical Center, Texas University, Dallas, Texas, USA \\ ${ }^{5}$ Department of Surgery, School of Medical Sciences, Universidade Estadual de Campinas (UNICAMP), Campinas, SP, Brazil
}

\begin{abstract}
Introduction: The obstructive sleep apnea (OSA) is caused by recurrent episodes of partial or total obstruction of the upper airway lasting more than 10 seconds during sleep. Laryngopharyngeal Reflux (LPR) is a variant of the disease Gastroesophageal Reflux that affects the larynx and pharynx.
\end{abstract}

Objectives: Evaluate the influence of obesity on the relationship between RFL and OSAS in patients with OSA.

Materials and Methods: An observational retrospective cross. We reviewed care protocol for patients with OSA that includes validated questionnaires for RFL as Sympton Reflux Index (RSI) and Reflux Finding Score (RSI).

Results: 105 patients were divided into obese group (39 patients) and non-obese patients (66 patients). In the evaluation of the mean RSI group of non-obese was similar between patients with mild OSA (11.96) and moderate (11.43). In the obese group the mean RSI was 6.7 in patients with mild OSA and 11.53 in patients with moderate to severe OSA $(\mathrm{p}<0.05)$.

Discussion: The sub-group of patients with OSA and RFL have several factors that promote inflammation of the upper airway. Patients with OSA should be screened and treated as the RFL increasing the quality of life.

Conclusion: The RFL are positively correlated and OSAS in obese patients.

\section{Effect of Chlorogenic Acid Administration on Glucemic Control in Patients with Intolerance Glucose}

\author{
Laura-Yareni Zuniga*, Esperanza Martinez-Abundis and Manuel Gonzalez-Ortiz \\ Institute of Experimental and Clinical Therapeutics, University of Guadalajara, Guadalajara, Mexico
}

\section{Abstract}

Introduction: The Chlorogenic acid has demonstrated promising effects on glucose, obesity, and dyslipidemia, among 
others research models, which could improve the glycemic control in patients with intolerance glucose.

Methods: A double-blind, randomized and placebo-controlled clinical trial, was carried out in 30 patients, with glucose intolerance, aged 30-60 years. The patients received $400 \mathrm{mg}$ of placebo or Chlorogenic acid capsules, three times daily before each meal for 90 days. We evaluated before and after intervention body weight (BW), body mass index (BMI), waist circumference (WC), fasting glucose (FG), 2-hour plasma glucose (2h-PG) after the Oral Glucose Tolerance Test (OGTT) of $75 \mathrm{~g}$, glycated hemoglobin A1C (A1C), triglycerides (TGs), total cholesterol (TC), high-density lipoprotein cholesterol (HDL-C), lowdensity lipoprotein cholesterol (LDL-C) and very-low density lipoprotein (VLDL). An Ethic Committee approved the protocol and a written informed consent was obtained from all volunteers. Statistical analysis: Fisher exact test, Wilcoxon signed rank test and Mann Whitney U test. Statistically significant $\mathrm{p} \leq 0.05$. Clinical trials.gov: NCT02621060.

Results: After Chlorogenic acid administration there were significant decrease in BW $(83.29 \pm 9.85$ vs. $80.77 \pm 11.01 \mathrm{~kg}$, $\mathrm{p}=0.020)$, BMI $\left(32.64 \pm 2.37\right.$ vs. $\left.31.40 \pm 2.71 \mathrm{~kg} / \mathrm{m}^{2}, \mathrm{p}=0.002\right), \mathrm{WC}(105.93 \pm 10.48$ vs. $103.93 \pm 10.01 \mathrm{~cm}, \mathrm{p}=0.170), \mathrm{FG}$ (5.36 \pm 0.47 vs. $5.23 \pm 0.32 \mathrm{mmol} / \mathrm{L}, \mathrm{p}=0.017)$, TGs $(1.58 \pm 0.65$ vs. $1.20 \pm 0.43 \mathrm{mmol} / \mathrm{L}, \mathrm{p}=0.002), \mathrm{TC}(4.54 \pm 0.65$ vs. 4.16 $\pm 0.71 \mathrm{mmol} / \mathrm{L}, \mathrm{p}=0.025), \mathrm{HDL}-\mathrm{C}(1.68 \pm 0.40$ vs. $1.90 \pm 0.24 \mathrm{mmol} / \mathrm{L}, \mathrm{p}=0.023), \mathrm{LDL}-\mathrm{C}(2.29 \pm 1.04$ vs. $2.29 \pm 1.04 \mathrm{p}=$ $0.049)$, VLDL ( $0.72 \pm 0.30$ vs. $0.55 \pm 0.20 \mathrm{mmol} / \mathrm{L}, \mathrm{p}=0.002)$.

Conclusion: Chlorogenic acid decreases BW, BMI,WC and improve the FG and lipid profile in patients with intolerance glucose.

\title{
References
}

1. McCarty MF. 2005. A chlorogenic acid-induced increase in GLP-1 production may mediate the impact of heavy coffee consumption on diabetes risk. Med Hypotheses 64(4): 848-853.

2. Renouf M, Marmet C, Giuffrida F, Lepage M, Barron D, et al. 2014. Dose-response plasma appearance of coffee chlorogenic and phenolic acids in adults. Mol Nutr Food Res 58(2): 301-309.

3. Meng S, Cao J. 2013. Roles of chlorogenic Acid on regulating glucose and lipids metabolism: A review. Evid Based Complement Alternat Med 8: 14571461.

\section{Transition from Metabolic Adaptation to Maladaptation of the Heart in Obesity: Role of Apelin}

\author{
Kunduzova $\mathrm{O}^{1,2^{*}}$, Alfarano $\mathrm{C}^{1,2}$, Foussal $\mathrm{C}^{1,2}$, Anesia $\mathrm{R}^{1,2}$, Valet $\mathrm{P}^{1,2}$, Parini $\mathrm{A}^{1,2}$ and Calise $\mathrm{D}^{3}$ \\ ${ }^{1}$ National Institute of Health and Medical Research (INSERM) U1048, Toulouse, Cedex 4, France \\ ${ }^{2}$ University of Toulouse, UPS, Institute of Metabolic and Cardiovascular Diseases, Toulouse, France \\ ${ }^{3}$ US006, Microsurgery Services, Toulouse, Cedex 4, France
}

\begin{abstract}
Impaired energy metabolism is the defining characteristic of obesity-related heart failure. The adipocyte-derived peptide apelin has a role in the regulation of cardiovascular and metabolic homeostasis and may contribute to the link between obesity, energy metabolism and cardiac function. Here we investigate the role of apelin in the transition from metabolic adaptation to maladaptation of the heart in obese state. Adult male C57BL/6J, apelin knock-out (KO) or wild-type mice were fed a high-fat $\operatorname{diet}(\mathrm{HFD})$ for 18 weeks. To induce heart failure, mice were subjected to pressure overload after 18 weeks of HFD. Long-term effects of apelin on fatty acid (FA) oxidation, glucose metabolism, cardiac function and mitochondrial changes were evaluated in HFD-fed mice after 4 weeks of pressure overload. Cardiomyocytes from HFD-fed mice were isolated for analysis of metabolic responses. In HFD-fed mice, pressure overload-induced transition from hypertrophy to heart failure is associated with reduced FA utilization, accelerated glucose oxidation and mitochondrial damage. Treatment of HFD-fed mice with apelin for 4 weeks prevented pressure overload-induced decline in FA metabolism and mitochondrial defects. Furthermore, apelin treatment lowered fasting plasma glucose, improved glucose tolerance and preserved cardiac function in HFD-fed mice subjected to pressure overload. In apelin KO HFD-fed mice, spontaneous cardiac dysfunction is associated with reduced FA oxidation and increased glucose oxidation. In isolated cardiomyocytes, apelin stimulated FA oxidation in a dose-dependent manner and this effect was prevented by small interfering RNA sirtuin 3 knockdown. These data suggest that obesity-related decline in cardiac function is associated with defective myocardial energy metabolism and mitochondrial abnormalities. Furthermore, our work points for therapeutic potential of apelin to prevent myocardial metabolic abnormalities in heart failure paired with obesity.
\end{abstract}




\title{
Relationship Between Recently Proposed Adiposity Indices and Inflammatory Markers in Elderly Women with Sarcopenic Obesity
}

\author{
Maurilio T. Dutra ${ }^{1^{*}}$, Andre B. Gadelha ${ }^{1}$, Ricardo M. Lima ${ }^{1}$ and Otavio T. Nobrega ${ }^{2}$ \\ ${ }^{1}$ College of Physical Education, University of Brasilia, Brazil \\ ${ }^{2}$ Faculty of Ceilandia, University of Brasilia, Brazil
}

\begin{abstract}
Aim: Reduction in fat free mass and increase in fat mass are well documented body composition alterations related to aging. The combination of these changes is a complex condition that has been named sarcopenic obesity (SO) [1]. Also, aging is associated with chronic inflammation [2]. Interleukin-6 (IL-6) and C-reactive protein (CRP) are inflammatory markers related to aging. However, the association between new adiposity indices [e.g. body adiposity index (BAI) and visceral adiposity index (VAI)] and inflammatory markers is poorly understood. The aim of this study was to investigate the association between BAI and VAI with inflammatory markers in elderly women with SO.

Methods: A total of 130 women $(66.7 \pm 5.2$ years $)$ underwent body composition analysis by dual energy X-ray absorptiometry (DEXA). Volunteers were classified according to SO definition [3]. BAI, VAI, and waist-to-hip ratio (WHR) were calculated as previously proposed [4,5]. Blood samples were collected for CRP, Tumor Necrosis Factor (TNF) and IL-6 measurements. Spearman and partial correlation tests were employed to analyze correlations.

Results: SO prevalence was 20.8\%. BAI correlated to DEXA-derived body fat content ( $\mathrm{rs}=0.90)$, CRP (rs $=0.55)$ and IL-6 ( $\mathrm{rs}=0.53$ ), whereas WHR correlated to CRP ( $\mathrm{rs}=0.60)$, all $\mathrm{p}<0.01$. After partial correlations adjusted for age, WHR correlated to $\mathrm{CRP}$ ( $\mathrm{rs}=0.62, \mathrm{p}<0.01$ ), whereas BAI presented statistical trend to correlate to $\mathrm{CRP}(\mathrm{rs}=0.35, \mathrm{p}=0.08)$ and TNF ( $\mathrm{rs}=0.38, \mathrm{p}=0.055$ ). VAI did not correlate to any of the inflammatory variables.

Conclusion: Simple and cheap anthropometric indices such as BAI and WHR may be better predictors of low-grade inflammation than VAI in elderly women with SO.

\section{References}

1. Jarosz PA, Bellar A. 2009. Sarcopenicobesity: an emerging cause of frailty in older adults. Geriatric Nursing 30(1): 64-70. doi: 10.1016/j. gerinurse.2008.02.010

2. Licastro F, Candore G, Lio D, Porcellini E, Colonna-Romano G, et al. 2005. Innate immunity and inflammation in ageing: a key for understanding agerelated diseases. Immun Ageing 2: 8. doi: 10.1186/1742-4933-2-8

3. Oliveira RJ, Bottaro M, Júnior JT, Farinatti PTV, Bezerra LA, et al. 2011. Identification of Sarcopenic Obesity in Postmenopausal Women: a cutoff Proposal. Braz J Med Biol Res 44(11): 1171-1176. doi: 10.1590/S0100-879X2011007500135

4. Bergman RN, Stefanovski D, Buchanan TA, Sumner AE, Reynolds JC, et al. 2011. A better index of body adiposity. Obesity 19(5): 1083-1089. doi: 10.1038/oby.2011.38

5. Amato MC, Giordano C. 2014. Visceral adiposity index: an indicator of adipose tissue dysfunction. International Journal of Endocrinology 1-7. doi: $10.1155 / 2014 / 730827$
\end{abstract}

\section{Featured Presentations III}

\section{Obesity and Liver Metabolic Profile in Zucker Rats: New Aspects and Approaches}

\author{
Stepan Melnyk ${ }^{1,2^{*}}$, Teresa Evans ${ }^{1,2}$, Oleksandra Pavliv ${ }^{1,2}$, Soheila Korourian ${ }^{3}$ and Reza Hakkak ${ }^{2-4}$ \\ ${ }^{1}$ Department of Pediatrics, University of Arkansas for Medical Sciences, AR, USA \\ ${ }^{2}$ Arkansas Children's Research Institute, AR, USA \\ ${ }^{3}$ Department of Pathology, University of Arkansas for Medical Sciences, AR, USA \\ ${ }^{4}$ Department of Dietetics and Nutrition, University of Arkansas for Medical Sciences, AR, USA
}

\begin{abstract}
The obesity epidemic in the United States and developed countries has been steadily rising in the past two decades. Obese populations have higher mortality rates from all cancers and other chronic diseases. Multiple experimental and clinical studies show that obesity can play an important role in promotion of fatty liver creating a major health problem in the USA and worldwide. Six weeks old lean $(n=26)$ and obese $(n=20)$ female Zucker rats were fed AIN-93 G diet and sacrificed 155 days later. Liver samples were frozen in liquid nitrogen and analyzed for metabolites content utilizing high sensitivity HPLC-ECD
\end{abstract}


and LC-MS techniques. Obese rats developed higher SAM $(\mathrm{P}<0.05)$ and SAM/SAH $(\mathrm{P}<0.03)$ and a lower $(\mathrm{P}<0.05)$ SAH level. Global DNA methylation $(5 \mathrm{mCyt})$ in obese rats was significantly $(\mathrm{P}>0.02)$ higher compared to lean rats. Levels of $\mathrm{GSH}$ were significantly $(\mathrm{P}<0.03)$ lower, GSSG level significantly $(\mathrm{P}<0.002)$ higher and GSH/GSSG ratio was significantly $(\mathrm{P}<0.005)$ lower in obese rats compared to lean rats. Additionally, DNA of obese group contained almost $25 \%$ higher $5 \mathrm{hmCyt}$ and over 23\% higher 8-OH-Gua. Both nitrosative stress biomarkers (3-nitroTyrosine and nitroso Glutathione) in obese rats were significantly $(\mathrm{P}<0.02)$ higher compare to lean rats. Obesity significantly affected metabolic processes in liver by creating both an oxidative and nitrosative stress with post-translational DNA modifications. Such an environment is capable of triggering irreversible metabolic/morphological changes in the liver of Zucker rats. Future studies are needed to better understand the relations between these complex fundamental processes, not only in liver, but in other vital organs for prevention of chronic pathological conditions and irreversible damages.

\title{
Maternal Exposure to the Production of Fireworks and Reduced Rate of New Onset Hypertension in Pregnancy
}

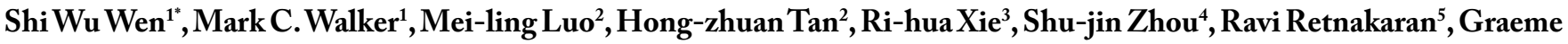 \\ Smith $^{6}$, Sandra T. Davidge ${ }^{7}$ and Jacquetta Trasler ${ }^{8}$
}

${ }^{1}$ Ottawa Hospital Research Institute, Canada

${ }^{2}$ Changsha Center for Disease Control and Prevention, China

${ }^{3}$ Central South University, China

${ }^{4}$ Hunan University of Medicine, China

${ }^{5}$ Liuyang Maternal and Child Hospital, China

${ }^{6}$ University of Toronto, Canada

${ }^{7}$ Queen's University, Kingston, Canada

${ }^{8}$ McGill University, Canada

\section{Abstract}

Background: Carbon monoxide (CO) is one of the main substances contained in fireworks. Previous studies suggested that $\mathrm{CO}$ may have protective effect on the development of hypertension of pregnancy.

Method: The authors conducted a prospective cohort study in Liuyang, Hunan, China between January 2010 and December 2011.Demographic and life-style variables of the participating pregnant women were obtained through structured interview with the women and clinical data were retrieved from antenatal medical records. Density of fireworks factories was defined as the number of fireworks factories per 1,000 residents in the township where the mothers resided during pregnancy. Multiple logistic regression analysis was used to analyze the independent association between maternal exposure to the production of fireworks and new onset hypertension in pregnancy.

Results: A total of 5,976 pregnant women were included in the final analysis. Density of fireworks factories was inversely correlated with incidence of new onset hypertension in pregnancy (Pearson correlation coefficient $=-0.29, \mathrm{P}<0.001$ ). Multiple logistic regression analysis showed that, compared with women who resided during pregnancy in a township with $0-0.25$ fireworks factories per 1,000 residents, the rates of new onset hypertension in pregnancy in women who resided in a township with 0.26-1.00 fireworks factories per 1,000 residents (Odds Ratio $=0.66,95 \%$ confidence interval: $0.46,0.96$ ) and $>1.5$ fireworks factories per 1,000 residents (Odds Ratio $=0.65,95 \%$ confidence interval: $0.44,0.97$ ) were reduced by more than $30 \%$.

Conclusion: Maternal exposure to the high density of fireworks factories is associated with reduced risk of developing new onset hypertension in pregnancy.

\section{Bile Acid Therapy for Obesity}

\section{Oren Tirosh}

Institute of Biochemistry, Food Science and Nutrition, The Hebrew University of Jerusalem, Rehovot, Israel

\section{Abstract}

Bile acids (BA) function as signaling molecules in the gastrointestinal tract to regulate glucose and energy metabolism. BA have been implicated to affect, satiety, lipid and cholesterol levels, glucose and energy metabolism, and the gut microbiome. TGR5, an emerging G protein-coupled receptor, was identified as a membrane receptor for BA. The expression of TGR5 and its function are distinct from the previously identified nuclear bile acid receptor, farnesoid X receptor (FXR). These two BA 
receptors complement with each other for maintaining homeostasis and mediating BA signaling. In 1975 Yosef and Fisher reported the presence of L-arginocholate in isolated perfused rat liver. The structure was confirmed by mass spectrometry analysis. Regular bile acids, as was analyzed in gallbladder, common ducts and duodenum are conjugated to glycine and taurine. The role of the lesser known basic amino acid bile acid conjugates is intriguing. One possibility is that these bile acids may serve as modulators to the effect of the regular BA. The aim of this study was to elucidate the effect of odd bile acids on non-alcoholic fatty liver disease, metabolic syndrome and obesity. Our results demonstrated that conjugation of primary bile acids with basic (positively charged at physiological $\mathrm{pH}$ ) and supplementation to mice protected the liver against high fat diet-induced fatty liver disease without any toxic effects, prevented the metabolic syndrome and attenuated the effect of diet-induced obesity. These results indicate that basic amino acids e.g., L-arginine and BA conjugates may be of potential therapy in metabolic diseases.

\title{
Cardiometabolic and Reproductive Benefits of Early Dietary Energy Restriction and Voluntary Exercise in an Obese PCOS-prone Rodent Model
}

\author{
Diane $A^{1,2^{*}}$, Kupreeva $\mathrm{M}^{1,2}$, Borthwick $\mathrm{F}^{1,2}$, Proctor $\mathrm{SD}^{1,2}$, Pierce $\mathrm{WD}^{1,2}$ and Vine $\mathrm{DF}^{3}$ \\ ${ }^{1}$ Metabolic and Cardiovascular Diseases Laboratory, Molecular and Cell Biology of Lipids Group, Alberta Diabetes, University of \\ Alberta, Edmonton, Alberta, Canada \\ ${ }^{2}$ Mazakowski Heart Institutes, Division of Human Nutrition, University of Alberta, Edmonton, Alberta, Canada \\ ${ }^{3}$ Department of Sociology, University of Alberta, Edmonton, Alberta, Canada
}

\begin{abstract}
Polycystic ovary syndrome (PCOS) is one of the most common endocrine-metabolic disorders in women of reproductive age characterized by ovulatory dysfunction, hyperandrogenism and cardiometabolic risk. The overweight-obese PCOS phenotype appears to have exacerbated reproductive dysfunction and cardiometabolic risk. In overweight-obese adult women with PCOS, exercise and energy restricted diets have shown limited and inconsistent effects on both cardiometabolic indices and reproductive outcomes. We hypothesized that an early lifestyle intervention involving exercise and dietary energy restriction to prevent or reduce the propensity for adiposity would modulate reproductive indices and cardiometabolic risk in an obese PCOS-prone rodent model. Weanling obese PCOS-prone and Lean-Control JCR: LA-cp rodents were given a chow diet ad libitum or an energy-restricted diet combined with or without voluntary exercise $(4 \mathrm{~h} / \mathrm{day})$ for 8 weeks. Dietary energy restriction and exercise lowered total body weight gain and body fat mass by $30 \%$ compared to free-fed sedentary or exercising obese PCOSprone animals $(\mathrm{P}<0.01)$. Energy restriction induced an increase in exercise intensity compared to free-feeding plus exercise conditions. Energy restriction and exercise decreased fasting plasma triglycerides and apoB48 concentrations in obese PCOSprone animals compared to free-fed and exercise or sedentary groups. The energy restriction and exercise combination in obese PCOS-prone animals significantly increased plasma sex-hormone binding globulin, hypothalamic cocaine-and amphetamineregulated transcript (CART) and Kisspeptin mRNA expression to levels of the Lean-Control group, and this was further associated with improvements in estrous cyclicity. The combination of exercise and dietary energy restriction when initiated in early life exerts beneficial effects on cardiometabolic and reproductive indices in an obese PCOS-prone rodent model, and this may be associated with normalization of the hypothalamic neuropeptides, Kisspeptin and CART.
\end{abstract}

\section{Effects of Beta-glucans Ingestion (Saccharomyces cerevisiae) on Metabolism of Rats Receiving High Fat Diet}

\author{
Luciano Jose Pereira ${ }^{*}$, Ticiana Vasques de Araujo ${ }^{2}$, Eric Francelino Andrade ${ }^{2}$, Raquel Vieira Lobato ${ }^{2}$, Nubia Ferreira \\ Gomes $^{2}$, Raimundo Vicente de Sousa ${ }^{2}$, Marcio Gilberto Zangeronimo ${ }^{2}$ and Debora Ribeiro Orlando ${ }^{3}$
}

${ }^{1}$ Federal University of Lavras- UFLA, Department of Health Sciences, Brazil

${ }^{2}$ Federal University of Lavras - UFLA, Department of Veterinary Sciences, Brazil

${ }^{3}$ Federal University of Jequitinhonba and Mucuri Valleys - UFVJM, Department of Agricultural Sciences, Brazil

\begin{abstract}
We investigated the effects of Beta-glucans (Saccharomyces cerevisiae) ingestion on metabolic parameters of Wistar rats receiving high fat diet. The experimental period was divided into two stages: in the first one, the animals were divided into two groups containing 12 animals each. The first group received commercial feed and the second received high fat diet containing $20 \%$ of pork fat during 60 days. At the end of this period, body weight, blood glucose and Lee index were assessed. In the second stage, those 24 animals were re-divided into four groups: $(\mathrm{C})$ - control diet; $(\mathrm{CB})$ - control diet and treated with Beta-glucan $(\mathrm{BG}) ;(\mathrm{O})$ - obese animals and $(\mathrm{OB})$ - obese animals treated with BG. Animals from groups $\mathrm{CB}$ and $\mathrm{OB}$ received $30 \mathrm{mg} / \mathrm{kg}$ of
\end{abstract}


BG dissolved in saline solution by gavage. Animals from groups $\mathrm{C}$ and $\mathrm{O}$ received only saline solution for 28 days. The design used was totally randomized in $2 \times 2$ factorial schemes. Data were submitted to analysis of variance (ANOVA). Animals from OB group showed inferior levels $(\mathrm{p}<0.05)$ of total cholesterol $(13.33 \%)$, triacylglycerols $(16.77 \%)$ and blood glucose $(23.97 \%)$ when compared to the animals from group $O$. The use of BG has provided smaller increase in Lee index $(p<0.05)$, without promoting alteration in feed and water consumption, organs weight, HDL-C, LDL+VLDL-C, carcass composition, villus/ crypt ratio, and pancreas, kidney and stomach histology. BG from Saccharomyces cerevisiae promoted beneficial metabolic effects in rats receiving high fat diet. This study was supported by FAPEMIG - Grant PPM 00268-14

\title{
Quantifying Parental Misconceptions of Childhood Obesity in California's Farm Worker Communities
}

\section{Banafsheh Sadeghi ${ }^{*}$, Lisa Martinez ${ }^{2}$, Rosa Gomez-Camacho², Alber Aguilera ${ }^{2}$, Mayra Munoz Gomez ${ }^{2}$, Iraklis Erik Tseregounis ${ }^{2}$, Linda Whent ${ }^{2}$, Adela de la Torre ${ }^{2}$, Sara Schaefer ${ }^{3}$ and Ulfat Shaikh ${ }^{4}$}

${ }^{1}$ University of California-Davis Department of Internal Medicine, CA, USA

${ }^{2}$ University of California-Davis, Center for Transnational Health, CA, USA

${ }^{3}$ University of California-Davis Foods for Health Institute, CA, USA

${ }^{4}$ University of California-Davis Department of Pediatrics, CA, USA

\begin{abstract}
Background: Ninos Sanos, Familia Sana is a childhood obesity prevention intervention study in Mexican-origin children in California's central valley. At baseline, data on socio-demographic status, health history, and anthropometric measures were collected.
\end{abstract}

Purpose: To a) assess the association between BMI z-score and related socio-demographic factors, b) explore the association between skinfold thickness and overweight/obesity status, and c) understand parental perception of their children's actual overweight/obesity status.

Methods: Using the baseline data from a five-year non-randomized and multifaceted intervention study, we analyzed data from 594 children (492 families) between 2 to 7 years old.

Findings: Forty-five percent of children and $82 \%$ of their mothers were overweight or obese. Ninety-two percent of children in the overweight and 53\% of children in the obese groups according to BMI were perceived as having normal weight by their mothers. Among anthropometric measures, waist circumference had the highest (0.713), and triceps skinfold thickness had the lowest (0.611) correlation with BMI z-score. Only in 33\% of overweight or obese children, either triceps or subscapular skinfold thickness was higher than $85^{\text {th }}$ percentile. In multivariate analyses, mothers' BMI and acculturation had positive association with children's BMI z-score.

Conclusions: Childhood obesity remains a major public health issue in Mexican-origin children in the US. Considering that the majority of the families are traditional or low-bicultural in this and similar communities, designing culturally sensitive interventions that impact the whole family rather than just the targeted age group which addresses the gaps in awareness as well as knowledge in healthy life behaviors is needed.

\section{Lipid Profiles in the Rural Community of North Kohala: The Kohala Health Study}

\author{
Kawika Liu*, Andrew Grandinetti and J Keawe'aimoku Kaholokula
}

John A Burns School of Medicine, HI, USA

\begin{abstract}
Objectives: Although the state of Hawaii enjoys relatively low cardiovascular disease (CVD) mortality, ethnic disparities persist and disproportionally affect native Hawaiians and Pacific Islanders. The Kohala Health Study (KHS) was conducted with the aim of estimating the prevalence of diabetes and other CVD risk factors in a multiethnic population in the rural community of North Kohala. Previous reports from the KHS focused on prevalence of Type 2 diabetes mellitus and cardiometabolic syndrome. The objective of this report is to compare the ethnic distribution of some other important CVD risk factors, namely low density lipoproteins (LDL), high density lipoproteins (HDL), and total cholesterol/HDL ratios (TC/HDL) in this population.
\end{abstract}

Methods: Cross-sectional data from 1,440 participants of Hawaiian, Japanese, Filipino, Caucasian, and mixed ethnic 
ancestries were analyzed for the study to include socio-demographics, anthropomorphic measurements, and biochemical markers. Body fat was estimated by calculating body mass indices (BMI), and body fat distribution by waist-hip ratios (WHR), and fasting plasma glucose and insulin levels were used to calculate insulin resistance using the Homeostasis Model (HOMAIR). Associations were estimated using general linear models (GLM).

Results: There were no significant age-adjusted ethnic differences in LDL cholesterol, however both HDL and TC/HDL were observed to vary significantly by ethnic group. Total cholesterol was highest among Filipino and compared to all other ethnic groups. While TC/HDL was highest among Hawaiians, the mean concentration was not significantly higher than observed among Japanese and Filipinos. After adjusting for BMI, ethnic differences continued to persist, however Filipinos appeared to have the highest TC/HDL. Further adjustment for leisure time physical activity further attenuated the ethnic differences in TC/HDL cholesterol, which were no longer statistically significant. The effect of physical activity is likely mediated by its effect on insulin sensitivity since its effect was no longer significant after adjustment for HOMA-IR.

Conclusions: Ethnic disparities in HDL and TC/HDL appear to parallel disparities in mortality in Hawaii. The disparity in HDL and TC/HDL were largely explained by differences in BMI and physical activity, mediated by their effects on insulin sensitivity.

\title{
Female Overweight and Obesity and Chronic Diseases in China
}

\author{
Cun-Xian Jia ${ }^{1^{*}}$, Li-Yuan $\mathrm{Liu}^{2}$ and Zhi-gang Yu ${ }^{2}$ \\ ${ }^{1}$ Department of Epidemiology, Shandong University School of Public Health, Jinan, China \\ ${ }^{2}$ Department of Breast Diseases, The Second Hospital of Shandong University, Jinan, China
}

\begin{abstract}
Objectives: In order to understand the relationship between female overweight and obesity and chronic diseases in China.

Methods: This study used the data about 120558 females aged 25-70 years old in eastern China. In this study, chronic diseases included breast cancer, benign breast tumors, hyperplasia of galactophone, cervix cancer, ovary cancer, ovary cyst, diabetes mellitus, hypertension, coronary heart disease. The overweight and obesity were classified according to body mass index (BMI) equal or higher than 25 and 30.
\end{abstract}

Results: The prevalence of female overweight was $22.18 \%$ while the obesity was $2.90 \%$. There were significant associations between BMI and breast cancer, hyperplasia of galactophone, cervix cancer, ovary cyst, diabetes mellitus, hypertension, coronary heart disease. After adjusting other potential confounding factors including age, education level, residence area, marriage status, and family income, overweight was still significantly associated with breast cancer $(\mathrm{OR}=1.658,95 \% \mathrm{CI}: 1.303-2.110)$, hyperplasia of galactophone $(\mathrm{OR}=1.158,95 \% \mathrm{CI}: 1.092-1.227)$, ovary cyst ( $\mathrm{OR}=1.300,95 \% \mathrm{CI}: 1.152-1.467)$, diabetes mellitus $(\mathrm{OR}=1.519,1.355-1.703)$, hypertension $(\mathrm{OR}=2.195,2.081-2.316)$, coronary heart disease $(\mathrm{OR}=1.252,1.122-1.397)$, obesity was still significantly associated with breast cancer $(\mathrm{OR}=2.478,95 \% \mathrm{CI}: 1585-3.875)$, diabetes mellitus $(\mathrm{OR}=2.217,95 \%$ $\mathrm{CI}: 1.828-2.688)$, hypertension $(\mathrm{OR}=.432,95 \% \mathrm{CI}: 4.025-4.881)$, coronary heart disease $(\mathrm{OR}=1.709,95 \% \mathrm{CI}: 1.418-2.061)$.

Conclusions: Overweight and obesity are important predictors for breast cancer, diabetes mellitus, hypertension and coronary heart disease in the females of China. Reducing weight might be an important measure for reducing the incidence of these diseases in the females of China.

\section{Black Tea Leaves and Rambutan Peel from Indonesia Natural Plant has Biological Function as Anti-Obesity Agents: Nutritional Genomic Study}

\section{Fatchyah Fatchiyah}

Department of Biology, Faculty of Mathematic and Natural Sciences, Brawijaya University, Malang, Indonesia

\begin{abstract}
Obesity is one global problem not only in developed countries, but also developing country. The number of obese people in Indonesia is increasing in line with the change of lifestyle. Nowadays, Indonesia entered into the top ten countries with developing overweight and obesity, around $40 \%$ of the adult population. This is alarming to become a major public health concern. Obesity is a complex disease causing lack energy balance in the body, genetics \& environmental factors, lifestyle and malnutrition. Indeed, Indonesia has a rich of natural plants such as tea leaves, rambutan peels, legumes, brown rice, fruits and vegetables. The ingredients of natural plants are able to control the developing obesity through the cell signaling pathway
\end{abstract}


which is organized at the level of proteins and biological active compounds and has ability to interact and modulate molecular mechanisms underlying an organism's physiological function. Our previous studies by animal model and in silico analysis shown the catechin of black tea leaves could lose the rat body weight gain and inhibit the $\mathrm{IgF}-1$ protein and $\mathrm{IgF}-1 \mathrm{receptor}$ interaction in the adipocyte. The ability of EC, EGC and EGCG of black tea was only prevented the L1-domain of IgF-1R binds of IgF1 protein and reduced the adipocyte proliferation. The rambutan peel extract (RPE) was also protected the receptor of IgF-1 interact with its ligand protein in obese rats, both expressions were lower than control rat group. RPE has an ability to down regulate directly the adipogenesis regulation of PPAR $\gamma$ gene expression and mRNA level of FABP4 genes and also prevent the body weight gain by the declining size of the adipocyte in an obese rat model, respectively. Conclusively, Catechin of black tea leaves and bioactive compound of rambutan peel extract has potential as an anti-obesity agent through regulation of the molecular signaling pathway of the adipogenesis gene cascade.

\title{
Laparoscopic Fundoplication with Sleeve Gastrectomy as a Potential Treatment of Morbidly Obese Patients with GERD, First Experience and Results
}

\author{
Kelimu Abudureyimu*, Aikebaier Aili, Yibitihaer Saifuding, Zhang Cheng, Pierdiwasi, Alimujiang Maitisiyiti and Wang \\ Zhi
}

Department of Laparoscopic Surgery, People's Hospital of Xinjiang Uyghur Autonomous Region, Urumqi, China

\begin{abstract}
Objective: To observe the efficacy of Laparoscopic fundoplication with sleeve gastrectomy.

Methods: The clinical data of 5 morbidly obese patients with GERD who underwent laparoscopic fundoplication with sleeve gastrectomy from February 2014 to October 2014 in People's Hospital of Xinjiang Uyghur Autonomous Region. After undergoing revised sleeve gastrectomy which reserve a small part of fundus, then underwent esophageal hiatal hernia repair and fundoplications which include Nissen fundoplication Toupet fundoplication and Dor fundoplication.

Result: All procedures were successfully performed via laparoscopy. Among 5 patients, 2 patients underwent with Nissen fundoplication, 2 with Toupet fundoplication, 1 has underwent with Dor fundoplication, and one also underwent lapascopic cholecystectomy. The mean operation time was $(156.8 \pm 26.7)$ minutes mean post-hospital stay was $(5.7 \pm 1.5)$ days. The follow up duration was 3-6 months, the mean weight lost was $(13.1 \pm 2.7) \mathrm{kg}$, $(25.7 \pm 3.8) \mathrm{kg}$ and $(37.3 \pm 4.1) \mathrm{kg}$, respectively, the percentage of the excess weight lost was $24.7 \% \pm 3.3 \%, 47.3 \% \pm 6.3 \%$ and $65.8 \% \pm 8.7 \%$, at 1,3 and 6 months following the procedure. After operation the symptoms of GERD were relieved in all patients. Esophagael high resolution manometry and acid measurement and endoscopy showed that 3 patients' erosive esophagitis were cured. Post-operative blood pressure and blood glucose level were back to normal in 3 patients who combined with impaired glucose tolerance and high blood pressure. Mild hair loss happened in 2 patients, mild anemia in 1 patient. No other complications were noticed.
\end{abstract}

Conclusion: Laparoscopic fundoplication with sleeve gastrectomy would be an acceptable option for treating morbidly obese patients combined with GERD and or hiatal hernia.

\section{Establishment of Laparoscopic Fundoplication with Sleeve Gastrectomy in a Porcine Model}

\author{
Aikebaier Aili, Kelimu Abudureyimu, Yibitihaer Saifuding, Zhang Cheng, Alimujiang Maisiyiti and Pierdiwasi Maimaiti \\ Department of Laparoscopic Surgery, People's Hospital of Xinjiang Uyghur Autonomous Region, China
}

\begin{abstract}
Objective: To study the feasibility and safety of laparoscopic fundoplication with sleeve gastrectomy in the porcine model.

Methods: A total of eight mini pigs underwent revised sleeve gastrectomy which reserved a small part of fundus, then underwent esophageal hiatal hernia repair and fundoplications including Nissen fundoplication, Toupet fundoplication, Dor fundoplication between December 2013 and May 2014 in Covidien Shanghai Clinical Training Center and Olympus (Shanghai) Laboratory. Intra-operative complications, main operative procedure, technical difficulties and cautions were recorded.

Results: Eight cases of sleeve gastrectomy with small part of reserved fundus and esophageal hiatal hernia repair and 24 cases of fundoplications were performed laparoscopically on eight mini pigs. Three types of fundoplications as Nissen, Toupet and Dor fundoplication were performed orderly on each mini pig. Vital signs of all animals were stable during the procedure. The mean operation time was $(20.2 \pm 13.7)$ minutes, and the mean blood loss was $(35.8 \pm 11.1)$ ml. There was no complication
\end{abstract}


except one liver damage. Operation time shorten significantly after practicing.

Conclusion: Establishment of laparoscopic fundoplication with sleeve gastrectomy in mini pig can be feasible and safe, and it can provide very helpful training opportunities for surgeons. The new surgical procedure would be an acceptable option for treating gastroesophageal reflux disease or hiatal hernia in morbidly obese patients.

\title{
Critical Roles of Long Non-Coding RNA's in Metabolic Homeostasis and Disease
}

\author{
Haiming $\mathrm{Cao}^{1 *}$, Ping $\mathrm{Li}^{1}$, Xiangbo Ruan ${ }^{1}$, Ling Yang ${ }^{1}$, Kurtis Kiesewetter $^{1}$, Andrew Cangelosi ${ }^{1}$, Haitao Luo $^{2}$, Yi Zhao ${ }^{2}$ and \\ Jun $\mathrm{Zhu}^{3}$ \\ ${ }^{1}$ Center for Molecular Medicine, NHLBI, National Institutes of Health Bethesda, MD, USA \\ ${ }^{2}$ Key Laboratory of Intelligent Information Processing, Institute of Computing Technology, Chinese Academy of Sciences, Beijing, PR \\ China \\ ${ }^{3}$ Systems Biology Center National Heart, Lung and Blood Institute, NIH, Bethesda, MD, USA
}

\begin{abstract}
Long non-coding RNAs (lncRNAs) constitute a significant portion of mammalian transcriptomes, yet their importance in metabolic physiology is largely unknown. To systematically identify functional lncRNAs regulating energy metabolism, we combine extensive genome wide screens, bio-informatics function predictions and cell-based analyses to identify 541 putative lncRNAs metabolic regulators in mice. We demonstrate a liver-enriched lncRNA we term liver-specific triglyceride regulator (lncLSTR) robustly regulates systemic lipid metabolism. Mice with a liver specific depletion of lncLSTR exhibit a marked reduction in postprandial plasma triglyceride levels. Bioinformatics analysis coupled with in vivo experimentation demonstrates that lncLSTR depletion activates a bile acid receptor, FXR-mediated pathway to enhance apoC2 expression, leading to robust lipoprotein lipase activation and increased plasma triglyceride clearance. We further show that lncLSTR depletion can be utilized therapeutically to reduce circulating triglyceride levels in a hyperlipidemia mouse model. Utilizing a similar approach, we identify an lncRNA that is robustly increased by the master transcription factor of lipogenesis, SREBP1cthus we termed lncSREBP. LncSREBP knockdown in mice increases the levels of plasma triglyceride and key lipogenic gene expressions in a SREBP1c-dependnet manner. Furthermore, we find the human homolog of lncSREBP exhibits similarly striking liver-specific expression and is under the robust regulation of SREBP1c in human cells. Importantly, human lncSREBP shows altered expression in patients of nonalcoholic fatty liver disease suggesting its role in the development of human steatosis. Taken together, our data support that a class of lncRNAs function as important metabolic regulators and provide a proof-of-principle that human lncRNAs could provide novel therapeutic targets for the treatment of metabolic disorders.
\end{abstract}

\section{Impact of Metabolic Syndrome on New-Onset Hypertension: Results from a Population-Based Survey Study}

\author{
Rongjing Ding ${ }^{*}$, Dayi Hu${ }^{1}$ and Zhugui Zhang ${ }^{2}$ \\ ${ }^{1}$ Peiking University Peoples' Hospital, China \\ ${ }^{2}$ Thomas Jefferson University, PA, USA
}

\section{Abstract}

Objective: Metabolic syndrome (MS), with different definitions, has been shown to contribute to risk of cardiovascular events in hypertension and has been associated with a wide range of cardiovascular risk factors. However, the association between MS and new-onset hypertension remains unclear.

Methods: We examined the association between MS and new-onset hypertension during a two-year period in a populationbased survey using random cluster sampling. The baseline survey was conducted in 10054 residents of four communities in north China between June and August of 2007; of these, 7222 residents completed follow up between June and August of 2009. Respondents were considered to have MS if they met three or more of the following criteria: 1) waist circumstance $\geq 90 \mathrm{~cm}$ in men and $\geq 85 \mathrm{~cm}$ in women; 2) triglyceride $\geq 150 \mathrm{mg} / \mathrm{dl}$ (1.7 mmol/l); 3) low HDL-C < $40 \mathrm{mg} / \mathrm{dl}$ in men and $<50 \mathrm{mg} / \mathrm{dl}$ in women; 4) high blood pressure > 130/85 mmHg; and 5) fasting glucose $>110 \mathrm{mg} / \mathrm{dl}$. A systolic blood pressure $\geq 140 \mathrm{mmHg}$ and/or diastolic blood pressure $\geq 90 \mathrm{mmHg}$, and/or use of antihypertensive medications within the two-year follow-up period was defined as new-onset hypertension.

Results: Among respondents who had normal blood pressure $(n=4034,55.86 \%)$ at baseline survey, $669(16.58 \%)$ had MS and 978 (24.24\%) developed hypertension over two years.MS was significantly associated with new-onset hypertension after 
adjustment for other known or suspected risk factors such as age, gender, clinical conditions and medications. The multivariate odds ratios for developing hypertension among participants who had MS, as compared with those who did not, were 3.21 (95\% confidence interval [CI], 2.57 to 4.03 , c-index $=0.78)$ and $4.11(95 \% \mathrm{CI}: 3.43$ to 4.92 , c-index $=0.72)$ for models with or without components of MS, respectively. Significant associations were also observed with odds ratios ranging from 2.5 to 4.1 ( $\mathrm{p}$-value < 0.01) when MS was calculated from definitions of World Health Organization and American Heart Association guidelines.

Conclusions: Metabolic syndrome was a potential independent risk factor of new-onset hypertension. his analysis can be applied to inform shared decision making between health care providers and patients.

\title{
Correlation Between Anthropometrics and Cardiorespiratory Function in Breast Cancer Survivors
}

\author{
Kyuwan Lee*, Nathalie Sami and Christina M. Dieli-Conwright \\ Women's Health and Exercise Laboratory, Division of Biokinesiology and Physical Therapy, University of Southern California, CA, USA
}

\begin{abstract}
Background: Obesity is a risk factor for cardiovascular disease (CVD) in breast cancer survivors (BCS). Screening for CVD involves assessing cardiorespiratory function (CRF), defined as the capability of the circulatory system to transport oxygen to tissues, and by measuring anthropometrics; elevated anthropometric measures such as high body mass index (BMI) or large waist circumference (WC) correlate with poor CRF. The purpose of this study was to determine the relationship between CRF and anthropometric measures including BMI, WC, waist-to-hip ratio (WHR) and waist-to-height ratio (WHtR) in BCS.
\end{abstract}

Methods: Seventy-seven BCS diagnosed with stage I-III breast cancer were included in this study. Height and weight were measured to calculate BMI. WC and hip circumference were measured to calculate WHR. WHtR was calculated as the ratio of WC and height. CRF was assessed using resting heart rate (RHR), and a 4-minute walk test to measure estimated VO2max. Pearson's correlations were used to determine the associations between anthropometrics and CRF.

Results: BMI, WC, and WHtR were significantly correlated with RHR and estimated VO2max $(\mathrm{p}<0.01)$. WHtR presented the strongest correlation with $\operatorname{RHR}(\mathrm{r}=.410, \mathrm{p}<0.01)$ followed by BMI $(\mathrm{r}=.379, \mathrm{p}<0.01)$, and WC $(\mathrm{r}=.361 \mathrm{p}<$ 0.01). Further, WHtR showed the strongest correlation with estimated VO2 max $(r=-.476, p<0.01)$, followed by BMI ( $r=$ $-.368, \mathrm{p}<0.01)$, WC $(\mathrm{r}=.295, \mathrm{p}<0.05)$ and WHR $(\mathrm{r}=-.398 \mathrm{p}<0.01)$.

Conclusion: WHtR is most closely associated with CRF compared to WC, BMI, and WHR. This study suggests that WHtR may identify BCS who are at increased risk for obesity-related declines in CRF.

\section{Adult Overweight and Obesity Among the Mongolian Populations}

\author{
Ouynbileg Janchiv*, Enkhtuya Palam, Ichinkhorloo Bonduush, Bolormaa Norov, Otgontuya Dugee and Tsogtbaatar \\ Byambaa
}

Public Health Institute of Ministry of Health, Mongolia

\begin{abstract}
Overweight and obesity are recognized as intermediate risk factors for NCDs. Trained staff performed anthropological measurements of weight, height and waist circumference in 5314 survey participants aged 15-64 years. The prevalence of overweight and obesity (BMI $\geq 25$ ) was $42.7 \%$ (95\% CI 39.9-45.5) in women and 37\% (95\% CI 33.0-41.0) in men. Proportion of overweight and obese people in 25-34 year age group was 2.2-2.5 times higher compared to 15-24 year age group. For instance, proportion of overweight and obese men aged 25-34 years was 40.1\%, which was 2.5 times more compared to $15-24$ year-old men (15.7\%). In persons aged 15-44 there was a statistically significant direct correlation between the prevalence of obesity and age in both genders. More than a half of populations aged 45-64 years were overweight or obese, and there were no significant differences between age groups. The prevalence of overweight and obesity was highest in 45-64 year-olds with $55.9-60.3 \%$ of men and $65.8-67.7 \%$ of women having BMI $\geq 25$. According to BMI risk assessment 39.8\% of populations were overweight or obese, with $27.3 \%$ overweight and $12.5 \%$ obese. Prevalence of overweight and obesity tended to increase with age, and the proportion of overweight or obese women in all age groups was higher compared to their male counterparts. Prevalence of central obesity was $29.1 \%$ in $15-64$ year-old men and $55.7 \%$ in women. Seven in ten women above the age of 35 were centrally obese.
\end{abstract}




\title{
Improving Consumption of Healthy Food Through a Technology-Dependent "Chef Coaching" Program
}

\author{
Rani Polak* and Edward M. Phillips \\ Institute of Lifestyle Medicine, Spaulding Rehabilitation Hospital, Harvard Medical School, Boston, MA, USA
}

\begin{abstract}
Background: Beneficial correlations have been observed between participation in food preparation and choosing healthier food. Prompted by this, Culinary Medicine (CM) programs delivering patient and physician curricula emphasizing skills such as shopping, food storage, and meal preparation are growing. These programs now typically offer either hands-on or visual culinary demonstrations in a teaching-kitchen setting. While these models may be the ideal, they face barriers for implementation including high costs. A remote "hands-off" culinary education is more accessible and affordable, and therefore a valuable option.
\end{abstract}

Methods: In 2014, a technology-dependent Chef Coaching Program, remotely delivered by a credentialed chef who is also a certified health coach, was developed at the Institute of Lifestyle Medicine, Joslin Diabetes Center, Harvard Medical School. It was comprised of coaching sessions via videoconference, including practical culinary skills such as meal planning, food purchasing and storage, and healthy food preparation techniques, delivered via an online CM resource center, including a collection of recipes, culinary video-clips and comprehensive culinary classes.

Results: (1) From 2014, the Chef Coaching program was offered at Harvard's Joslin Diabetes Center to healthy individuals and patients with overweight, obesity, and diabetes (type $1 \& 2$ ). Pilot outcomes ( $=5$; twelve 30-minute coaching sessions) include improved culinary attitude and confidence, nutritional consumption, and HgA1c. (2) From 2015, we coached Yale Preventive Medicine residents ( $n=4$; six 45-minute coaching sessions), who reported heightened confidence in helping patients improve their lifestyle-related behaviors. (3) In 2015, we trained credentialed chefs who are also certified health and wellness coaches ( $\mathrm{n}=7$; eight 90-minute training); all currently practice the Chef Coaching Program as part of their internship in seven North American coaching practices (data pending). (4) Our next step is to train chefs without any coaching experience (grant approved).

Conclusion: This technology-dependent educational initiative is expected to have important nutritional education implications by efficiently and effectively educating both health and culinary providers and patients. We hope that it will be the gateway for chefs to be key agents in improved nutrition.

\section{Role of CTHRC1 in Adipose Tissue Formation, Body Composition, Physical Activity and Endurance Athletic Performance}

\author{
Volkhard Lindner ${ }^{*}$, J. Patrizia Stohn ${ }^{1}$, Matthew E. Siviski ${ }^{1}$, Yong-Ri Jin ${ }^{1}$, Qiaozeng Wang ${ }^{1}$, Victoria DeMambro ${ }^{1}$, Lucy \\ Liaw $^{1}$, Clifford J. Rosen ${ }^{1}$, Igor Prudovsky ${ }^{1}$, Elaine C. Lee ${ }^{2}$ and Frank Suhr ${ }^{3}$
}

${ }^{1}$ Maine Medical Center Research Institute, ME, USA

${ }^{2}$ Department of Kinesiology, University of Connecticut, CT, USA

${ }^{3}$ Deutsche Sporthochschule Köln, Germany

\begin{abstract}
CTHRC1, collagen triple helix repeat containing-1, was discovered in our laboratory and recently we characterized its function as a hormone expressed in the hypothalamus and pituitary gland. CTHRC1 null mice were generated to investigate the effects of loss of CTHRC1 on metabolism, voluntary physical activity, and grip strength. Compared to wild types, CTHRC1 null mice had similar body weights but increased fat mass and decreased muscle mass. In vitro studies demonstrated that CTHRC1 inhibited adipocyte differentiation as well as PPAR and CREB reporter activity, while preadipocytes isolated from CTHRC1 null mice exhibited enhanced adipogenic differentiation. Voluntary physical activity in CTHRC1 null mice as determined by wheel running was reduced to approximately half the distance covered by wild types. Reduced grip strength was observed in Cthrc1 null mice at the age of 15 weeks or older with reduced performance and mass of fast twitch muscle. In approximately $75 \%$ of healthy human subjects CTHRC1 plasma levels are below detection $(<30 \mathrm{pg} / \mathrm{ml})$, whereas the vast majority of endurance athletes were found to have high CTHRC1 plasma levels, up to $100 \mathrm{ng} / \mathrm{ml}$ in ultramarathon runners. Finding increased resting energy expenditure in Cthrc1 null mice and high CTHRC1 levels in endurance athletes suggests a potential role for circulating CTHRC1 regulating metabolic efficiency. Preliminary data suggest that circulating CTHRC1 levels may be genetically determined with CTHRC1 produced by the hypothalamus and released by the posterior pituitary. Consistent with a hormone, our data indicate that CTHRC1 has multiple functions including inhibition of adipogenesis,
\end{abstract}


regulation of voluntary physical activity and metabolic efficiency.

\title{
Improvements in Glucose Tolerance with Bikram Yoga in Older Obese Adults: A Pilot Study
}

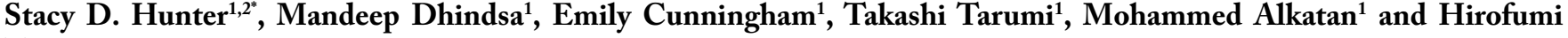 \\ Tanaka ${ }^{1}$
}

${ }^{1}$ University of Texas at Austin, TX, USA

${ }^{2}$ Pure Action, Inc., Austin, TX, USA

\begin{abstract}
Bikram yoga is an exotic form of physical activity combining hatha yoga and thermal therapy that could positively impact metabolic health. Although this increasingly popular alternative exercise may be ideal for obese adults due to its low impact nature, few studies have elucidated the health benefits associated with it. As an initial step, we determined the effect of Bikram yoga on glucose tolerance. Fourteen young lean and 15 older obese subjects completed an 8-week Bikram yoga intervention in which classes were completed 3 times weekly. Glucose tolerance was assessed using a $75 \mathrm{~g}$ oral glucose tolerance test. The area under the glucose curve following the oral glucose tolerance test was significantly reduced as a result of the Bikram yoga intervention in older obese $(\mathrm{P}<0.05)$ but not in young lean subjects. We concluded that a short-term Bikram yoga intervention improved glucose tolerance in older obese, but not in young lean adults.
\end{abstract}

\section{Single-blind, Placebo Controlled Randomized Clinical Trial of Chitosan for Body Weight Reduction}

\author{
Veronique Maquet ${ }^{1^{*}}$, Audrey Deschamps ${ }^{1}$, Milan C. Satia ${ }^{2}$ and Vandit R. Trivedi ${ }^{2}$ \\ ${ }^{1}$ KitoZyme, Rue de Milmort 680, 4040 Herstal, Belgium \\ ${ }^{2}$ Ethicare Clinical Trial Services, Titanium City Centre, Abmedabad, India
}

\begin{abstract}
Chitosan is a dietary fiber obtained by deacetylation of chitin, the second most abundant natural polysaccharide after cellulose. Thanks to its exceptional fat binding properties, chitosan is being used as an efficient product for treatment of overweight and obesity. In a recent clinical study, the efficacy and safety of fungal chitosan derived from Aspergillus niger has been evaluated for the treatment of excess weight in absence of dietary restrictions or changes. This randomized, single-blind, placebo-controlled clinical study was conducted in 96 overweight and obese subjects receiving either $2.5 \mathrm{~g} / \mathrm{day}$ of chitosan or placebo for 90 days. The mean change in body weight at day 45 and day 90 was $-1.78 \pm 1.37 \mathrm{~kg}$ and $-3.10 \pm 1.95 \mathrm{~kg}$, respectively, in patients receiving chitosan which was significantly different as compared to placebo $(\mathrm{p}<0.0001)$. In addition, BMI was decreased by 10.91 fold compared to placebo after 90 days of treatment with chitosan. Simultaneously, reduction in body composition and anthropometric parameters together with improvement in QoL score were observed. Chitosan was also able to reduce $\mathrm{HbA} 1 \mathrm{C}$ level in subjects with initial higher value. The mean caloric intake shows that there was no change of food habits for both groups. Lipid levels were unaffected. Adverse events were mild and unrelated to study treatment. In conclusion, this clinical study shows that chitosan from fungal origin is able to efficiently and safely reduce the mean body weight up to 3 $\mathrm{kg}$ after a 90-days period in overweight and obese subjects.
\end{abstract}

\section{Bite Force Difference among Obese Adolescents in Central Taiwan}

\author{
Kuo-Ting Sun ${ }^{1,2^{*}}$, Shih-Chueh Chen ${ }^{3}$, Yu-Fen Li ${ }^{4}$, Hsien-Hsiung Chiang ${ }^{5}$, Chi-Yuan Li ${ }^{6}$ and Ming-Gene Tu ${ }^{7}$ \\ ${ }^{1}$ Department of Pediatric Dentistry, China Medical University Hospital, No.2Yu-Der Rd., Taichung, Taiwan \\ ${ }^{2}$ School of Dentistry, China Medical University, No.91 Hsueb-Shih Rd, Taichung, Taiwan \\ ${ }^{3}$ Department of Endocrinology, Cheng Ching Hospital, No.139 Ping-Tien St., Taichung, Taiwan \\ ${ }^{4}$ Institute of Biostatistics, China Medical University, No.91 Hsueh-Shih Rd, Taichung, Taiwan \\ ${ }_{5}^{5}$ epartment of Pediatric Dentistry, China Medical University Hospital, No.2Yu-Der Rd., Taichung, Taiwan \\ ${ }^{6}$ Department of Anesthesiology, China Medical University Hospital, No. 2 Yu-Der Rd, Taichung, Taiwan \\ ${ }^{7}$ School of Dentistry, China Medical University, No.91 Hsueh-Shih Rd, Taichung, Taiwan
}




\title{
Abstract
}

Background/Purpose: Maximal bite force of the jaw can cause thorough food chewing and result good digestion. Bite force is related to the health of the masticatory muscles. Muscle force is frequently affected by obesity in adolescence, but little is known about how obesity influences the maximum bite force and the difference between genders.

Methods: Five hundred and seventy-seven adolescent students (292 girls and 285 boys) aged 13 to 16 years were recruited for the cross-sectional study from central Taiwan in 2009. The maximum bite force, hand strength, triceps skin-fold fat thickness, the serum level of testosterone and Body Mass Index (BMI) were measured. Dental health was evaluated based on malocclusion and dental caries.

Results: The prevalence of obesity was two-fold higher in boys (14.39\%) than in girls (7.88\%). The bite force in girls was highest in the obese group $(32.49 \pm 19.13 \mathrm{~kg}$, mean \pm standard deviation), whereas in boys it was higher in the over weighted group $(41.89 \pm 19.3 \mathrm{~kg})$ than in the obese group $(33.21 \pm 17.12 \mathrm{~kg})$. The mean serum level of testosterone increased with BMI in girls $(p=0.0172)$, while it decreased with BMI in boys $(p=0.0014)$. The trends of serum testosterone and bite force with BMI were similar in the two genders.

Conclusion: The maximum bite force decreased in obese boys but increased in obese girls, which may be due to the sensitivity to testosterone being modulated by the fat level.

\section{Abdominal Obesity in Recyclable Waste Pickers}

\author{
Flavia Auler ${ }^{*}$, Alika T. A. Nakashima ${ }^{1}$ and Roberto K. N. Cuman ${ }^{2}$ \\ ${ }^{1}$ Pontifical Catholic University of Parana, Curitiba, PR, Brazil \\ ${ }^{2}$ State University of Maringa, Maringa, PR, Brazil
}

\begin{abstract}
The goal of this study was to determine the prevalence of abdominal obesity (AO) in a population of recyclable waste pickers and to ascertain its association with various risk factors. This aim was achieved through a descriptive cross-sectional study on 273 pickers from the South of Brazil. The outcome criterion used was waist circumference (WC), with a cut-off value as established by the International Diabetes Federation (2009). The exposure variables were sociodemographic, anthropometric and biochemical. Most of the participants were adults (92.3\%), women (72.5\%), non-whites (58.6\%), overweight people (51.3\%), hypertensive people (25\%) and short people (21.6\%). The prevalence of $\mathrm{AO}$ was high $(57.5 \%)$ and was associated with female gender (OR 4.35) and differences in total cholesterol (OR 2.02), low density lipoprotein (LDL) fraction (OR 4.24), blood glucose (OR 3.84), Castelli indices I (OR 2.72) and II (OR 2.45), waist-to-height ratio (OR 91.2), conicity index (OR 12.1) and body fat $(\mathrm{OR} 89.5)(\mathrm{p}<0.05)$. The data show a high prevalence of $\mathrm{AO}$ in this vulnerable population with many risk factors. Public policies on income and education targeting this population should be expanded to improve its health profile.

\section{References}

1. Martins IS, Marinho SP. 2003.The potential of central obesity anthropometric indicators as diagnostic tools. Revista de Saúde Pública 37(6): 760-767. doi: $10.1590 / \mathrm{S} 0034-89102003000600011$

2. Monteiro CA, Moura EC, Conde WL, Popkin BM. 2004. Socioeconomic status and obesity in adult populations of developing countries. Bulletin of the World Health Organization 82(12): 940-946. doi: 10.1590/S0042-96862004001200011

3. Oliveira Lde P, Pereira ML, Azevedo A, Lunet N.2012. Risk factors for cardiovascular disease among the homeless and in the general population of Portugal. Cadernos de Saúde Pública 28(8): 1517-1529. doi: 10.1590/S0102-311X2012000800010

4. Dall'agnol CM, Fernandes DS. 2007. Health and self-care among garbage collectors: work experiences in a recyclable garbage cooperative. Revista Latino-Americana de Enfermagem 15: 729-735. doi: 10.1590/S0104-11692007000700003
\end{abstract}

\section{CALHM1 Deletion in Mice Affects Taste Nerve Responses, Food Intake, Body Weight and Life Span}

\section{Eric Kaplan* and Goran Hellekant}

Department of Biomedical Sciences, Medical School, University of Minnesota, Duluth, MN, USA

\section{Abstract}

Stimulation of Type II taste receptor cells (TRC) with T1R taste receptors causes sweet or umami taste, while T2Rs elicit bitter taste. Type II TRCs contain the calcium channel, CALHM1, which releases ATP transmitter to taste fibers. We have 
previously demonstrated with chorda tympani (CT) nerve recordings and two-bottle preference (TBP) tests that mice with genetically deleted Calhm1 (KO) have severely impaired perception of sweet, bitter and umami compounds, while their sour and salty tasting ability is unaltered. Here we present data from $\mathrm{KO}$ mice of effects on glossopharyngeal nerve (NG) responses, TBP, food intake, body weight (BW) and life span. KO mice have no NG response to sweet and a suppressed response to bitter compared to control (WT) mice. KO mice showed some NG response to umami, suggesting that umami taste involves both CALHM1 and non-CALHM1 modulated signals. NG responses to sour and salty were not significantly different between $\mathrm{KO}$ and WT mice. Behavioral data conformed in general with the NG data. Adult KO mice consumed less food, weighed significantly less and lived almost a year longer than WT mice. Our more recent results show a significant positive correlation between BW and resting blood glucose levels in both KO and WT mice. Taken together these data demonstrate that sweet taste majorly influences food intake, body weight and life span.

\title{
An OPEN Discussion of How Eating Behaviors Manifest in Low-Income Women and Their Preschool-Aged Children
}

\author{
Lenwood W. Hayman ${ }^{1 *}$, Nipher M. Malika ${ }^{2}$, Hannah J. Lee ${ }^{3}$, Alison L. Miller ${ }^{3}$ and Julie C. Lumeng ${ }^{3}$ \\ ${ }^{1}$ University of Michigan-Flint, MI, USA \\ ${ }^{2}$ Loma Linda University, CA, USA \\ ${ }^{3}$ University of Michigan, MI, USA
}

\begin{abstract}
Emotional-eating has been proposed as a risk factor for obesity. However, it is unclear as to how individuals conceptualize this behavior. As a result, it is also unclear as to how this behavior relates to comfort food seeking, food cravings, and food addiction. We sought to understand how women conceptualize emotional-eating and how this conceptualization may manifest in seeking comfort foods, food cravings, and food addiction. Sixty-one low-income women with preschool-aged children (ages 2-5 years) participated in either a focus group or individual semi-structured interview during which they were asked about their conceptualizations of eating behaviors among adults and children. Responses were audio-recorded and transcribed. Themes were identified using the constant comparative method of qualitative analysis. Identified themes reveal that participants view emotional-eating as uncommon, severe, pitiable, and a reflection of a lack of self-control. Additionally, when this behavior occurred among children, it resulted from neglect or even abuse. Additional themes revealed that respondents believed that adults and children eat comfort foods in response to a variety of emotional causes - both positive and negative, and that food cravings were common and less severe than food addiction. Future research and interventions should seek to develop more detailed conceptualizations of these behaviors to improve measurement, destigmatize emotional-eating, and potentially capitalize on the strong identification of comfort food seeking, food craving, and food addiction by targeting these behaviors for interventions.
\end{abstract}

\section{Impact of Migration on Non Communicable Diseases and Risk Factors}

\author{
Shamim Begam Neduvenchery \\ Al-Zora Health Care Center, United Arab Emirates
}

\section{Abstract}

Introduction: The objective of the study was to compare the magnitude of non-communicable disease (NCD) risk factors between gulf migrant workers and non-migrant workers of Malappuram district, Kerala.

Methods: Using a multistage cluster sampling technique, 384 adult males between 25-65 years of age were selected. Data on NCD risk factors were collected using World Health Organization (WHO) STEPS protocol. Data were analyzed using SPSS software.

Results: Prevalence of current tobacco use was $21.4 \%$ among migrants and $16.6 \%$ among non-migrants, current alcohol use was $8.9 \%$ among migrants and $12.4 \%$ among non-migrants, physical inactivity was $26.7 \%$ among migrants and $23.8 \%$ among non-migrants, poor diet habit was $86.9 \%$ among migrants and $76.2 \%$ among non-migrants, history of chronic diseases was $37.5 \%$ among migrants and 21\%among non-migrants, working 7 days/week was $35 \%$ among migrants and $1 \%$ among non-migrants, working $>8$ hrs/day was $76.9 \%$ among migrants and 33.1\% among non-migrants, sleeping less than 6 hrs/day was $41.3 \%$ among migrants and $14 \%$ among non-migrants. Prevalence of hypertension was $59.7 \%$ among migrants and $29.8 \%$ among non-migrants $(\mathrm{p}=<0.001$ ) (adjusted OR 2.5, 95\% CI $=1.38-4.46$ ), abdominal obesity was $79.5 \%$ among migrants and 
44.5\% among non-migrants ( $\mathrm{p}=<0.001$ ) (adjusted OR 2.4, 95\% CI = 1.35-4.31). Among migrant hypertensives $43.5 \%$ were aware, $33.9 \%$ were on treatment and $12.2 \%$ achieved adequate control, the corresponding figures for non-migrants were $56.9 \%$, $53.4 \%$, and $48.3 \%$ respectively.

Conclusion: Majority of the risk factors were significantly higher among gulf migrants than non-migrants, which calls for urgent intervention to reduce these risk factors in this migrant population.

\title{
Incorporating the Health Belief Model into Preventative Health Programs to Improve Participant Adherence and Outcomes
}

\author{
Victor Romano \\ Johnson C. Smith University, NC, USA
}

\begin{abstract}
While healthcare cost continues to rise worldwide, the prevalence of modifiable risk behaviors and poor lifestyle habits are also rising. The leading causes of death continue to be attributed to unhealthy lifestyle choices. Many of these health problems associated with unhealthy lifestyle choices, such as hypertension, obesity, and diabetes are increased for low-income minority populations. The Health Belief Model (HBM) is one of the most widely recognized conceptual frameworks for creating healthy behaviors by focusing on positive behavioral change at the individual level. The HBM is designed to assist in explaining and predicting preventive health behavior. It focuses on the individual's motivation to undertake a health behavior and can be divided into three main categories: individual perceptions, modifying behaviors, and likelihood of action. It is important to shape and change the culture of the individual. In order to create change, there must develop a strong motivational reason that outweighs the trouble and turbulence associated with giving up old habits. Most obesity and chronic disease programs are usually 8-12 weeks in length. However, regardless of the positive behavior changes that might have happened during that time, the highest rate of relapse is seen early after the end of the program. Successful integration of the HBM into these programs can lead to the reduction of behavioral relapse, assisting in improving participants' adherence and outcomes. This session will describe the HBM and how to successfully integrate the HBM into a variety of preventative health programming, focusing on obesity and chronic diseases.
\end{abstract}

\section{Resistin and Antioxidant: Are They Predictors of Disease Severity and Outcome in Childhood Asthma}

\author{
Eitedal Daoud ${ }^{*}$, Maha M. Saber ${ }^{1}$, Reda M. Daoud $^{2}$, Khaled G. Abdel-Wahhab ${ }^{3}$ and Lobna Saber ${ }^{4}$ \\ ${ }^{1}$ Complementary Medicine Department, National Research Centre, Egypt \\ ${ }^{2}$ E.N.T Department, Faculty of Medicine, Al Azhar University, Egypt \\ ${ }^{3}$ Medical Physiology Department, National Research Centre, Egypt \\ ${ }^{4}$ Biochemistry Department, Faculty of Medicine, Al Azhar University, Egypt
}

\section{Abstract}

Background: Asthma is a chronic inflammatory lung disease involving complex interactions between numerous cell types and mediators that result in airflow limitation, hyperreactivity, and remodeling. There is a link between the increase in reactive oxygen (ROS) and the asthma severity. Also, resistin is suggested to play a role in modulating asthma risk and/or severity.

Aim of Work: To evaluate level of resistin, antioxidant and IL-1 in atopic asthmatic children and correlate their levels to asthma severity and disease outcome.

Methodology: Eighty-three atopic asthmatics (AA) children and 30 non-asthmatics healthy controls, aged 6-12 years were studied. (AA) group included with positive skin prick test. Blood levels of resistin, IL-1, total anti-oxidant capacity (TAC), reduced glutathione enzyme (red GSH), paraooxnase-1 (PON-1) and nitric oxide (NO) as an end product of lipid peroxidation were measured.

Results: There were reduction with significant differences in levels of resistin $(p \leq 0.055)$, activity of TAC ( $p \leq 0.011)$, PON-I ( 150.005$)$, and increased NO ( $\leq 0.007)$ and IL-1 $(p \leq 0.000)$ however, there was no significant difference in level of redGSH $(\mathrm{P} \leq 0.980)$ between AA and healthy controls. There was significant negative correlation between resistin, red GSH, TAC and PON-I level and severity of illness, and a significant positive correlation of NO and IL-1 between their levels and severity of the disease. No significant correlation was detected between resistin and IL-1 apart from significant correlation 
between it and TAC ( $\mathrm{r}=0.96, \mathrm{p} \leq 0.001)$, PON1 $(\mathrm{r}=0.969, \mathrm{p} \leq 0.001), \mathrm{NO}(\mathrm{r}=0.673, \mathrm{p} \leq 0.000)$ and red GSH $(\mathrm{r}=0.889, \mathrm{p} \leq 0.000)$.

Conclusions: Our findings suggest that: resistin, and antioxidant may serve as predictors of disease severity in childhood $\mathrm{AA}$, and proved their role in the pathogenesis of airway inflammation. Their close associations with diseases severity, may have a disease-modifying effects in the outcome of childhood asthma.

\title{
Effect of Laser Lipolysis Therapy as A Noninvasive Approach on Adipose Tissue Thickness in Egyptian Women
}

\author{
Maha Saber ${ }^{*}$, Said Shalaby ${ }^{1}$, Amira Medhat ${ }^{1}$, Ahmed Kharbotly ${ }^{2}$, Nashwa Taher ${ }^{3}$ and Lobna M. Saber ${ }^{4}$ \\ ${ }^{1}$ Complementary Medicine Department, National Research Center, Cairo, Egypt \\ ${ }^{2}$ National Institute of Laser, Cairo University, Egypt \\ ${ }^{3}$ Rheumatology Department, Kasr Al-Aini-Cairo University, Egypt \\ ${ }^{4}$ Medical Biochemistry Department, Faculty of Medicine-Al Azhar University for Girls, Egypt
}

\begin{abstract}
The prevalence of overweight and obesity is increasing worldwide. Obesity is a complex disorder involving an excessive amount of body fat. Obesity isn't just a cosmetic concern. It increases the risk of diseases and health problems, such as heart disease, diabetes and high blood pressure. The main objective of this study was to measure the effect of Laser Lipolysis on adipose tissue thickness in women. A randomized, triple-blind, placebo-controlled trial of noninvasive Laser Lipolysis therapy was carried out on obese women those attended at complementary medicine clinic, center of excellent, National Research Center, Egypt. Sixty volunteer women of age between 30-50 years with a body mass index $25-35 \mathrm{~kg} / \mathrm{m}^{2}$ with buttock obesity (waist hip ratio less than 0.8 ) were included. All participants were undergone a balanced healthy diet for weight maintenance program. They were randomly assigned into two groups: 1 ) intervention group that receive Laser Lipolysis treatment, and 2) matching control group that receive a sham treatment; both for twice a week for 6 weeks. Local reduction of subcutaneous fat was measured by ultrasonography. The results revealed a significant decrease in fat thickness associated with a significant increase in waist hip ratio among women of intervention group after Laser Lipolysis treatment and weight maintenance program compared to control group. There were no significant differences between two groups regarding serum lipid profile including total cholesterol, HDL and LDL levels as well as weight loss at the end of the study. In conclusion Laser lipolysis is a safe, effective therapy for reduction of fat tissue thickness and well-tolerated noninvasive procedure for body contouring.
\end{abstract}

\section{$\underline{\text { Poster Presentations }}$}

\section{Maternal Diet Effects During Perinatal Period on Cognitive and Hedonic Brain Process and Behavior of Adult Age Yucatan Minipigs}

\author{
Yentl Gautier ${ }^{*}$, Isabelle Luneau ${ }^{1}$, Nicolas Coquery ${ }^{1}$, Paul Meurice ${ }^{1}$, Regis Janvier ${ }^{1}$, Julien Georges ${ }^{1}$, David Val-Laillet ${ }^{1}$, \\ Charles-Henri Malbert ${ }^{2}$ and Mickael Genissel ${ }^{3}$
}

${ }^{1} I N R A$, UR1341 ADNC, France

${ }^{2}$ INRA, US 1395 AniScans, France

${ }^{3}$ INRA, UMR 1348 PEGASE, France

\begin{abstract}
A lot of evidences defend the existence of a relationship between industrial food consumption and the increase of obesity prevalence (OMS, 2011). Particularly, the quality of early nutrition has long-term impact on the offspring phenotype and health status (Barker, 1989; Rice et Barone, 2000).

Our study explored the impact of Western diet early exposure on both cognitive and hedonic functions through behavioural assessments and brain imaging, to determine the role of maternal diet (High Fat Fructose diet, HFF versus standard diet, STD) during pregnancy and lactation on the cognitive abilities and eating behaviour of the progeny at the adult age, i.e. $\mathrm{xx}$ males and females Yucatan minipigs.

Learning and memory performances were tested using the holeboard discrimination task, with a palatable food reward $\left(M \& M{ }^{\circledR}{ }^{\circledR}\right)$, and alley maze. Eating behaviour was explored with a two-choice feed test and an operant conditioning test with progressive ratio. In parallel, the brain basal glucose metabolism was investigated using Positron Emission Tomography (PET)
\end{abstract}


and the dopaminergic brain reward system (in particular the striatum) was explored using DAT---scan imaging.

We show that early nutrition can indirectly impact cognitive performances, increasing motivation for reward, and decreasing attention level and exploratory behaviour in the HFF--- exposed group. Modulation of the emotional status in an anxiogenic situation suggests that early nutrition can condition motivational and attentional functions that affect learning processes, and potentially smooth differences between the two groups. Brain sub--- activations and less expression of dopamine transporter (DAT) observed in HFF animals in the anterior prefrontal cortex and nucleus accumbens are consistent with this hypothesis.

\title{
Phthalate Exposure and Metabolic Parameters in Korean Girls
}

\author{
Shin Hye Kim ${ }^{1}$, Hyunwook Nam$^{2}$ and Heesoo Pyo ${ }^{3}$ \\ ${ }^{1}$ Department of Pediatrics, Sanggye Paik Hospital, Inje University College of Medicine, Seoul, Republic of Korea \\ ${ }^{2}$ The Hill School, PA, USA \\ ${ }^{3}$ Molecular Recognition Research Center, Korea Institute of Science and Technology, Seoul, Republic of Korea
}

\begin{abstract}
Objectives: We studied to examine the associations of urine levels of phthalate metabolites with obesity and metabolic parameters in Korean girls.

Methods: A total of 139 girls (67 overweight cases and 72 controls, aged 6 to $13 \mathrm{yr}$ ) were recruited. Spot urine samples were collected and phthalate monoesters were analyzed using gas chromatography-mass spectrometry (GC-MS). Associations between phthalate exposure and metabolic parameters were examined by multiple linear regression and Logistic regression analyses.

Results: There was no significant difference in the concentrations of all phthalate monoesters between overweight and control girls, however, percentage fraction of MEOHP among DEHP metabolites (MEOHP\%) were significantly lower in overweight girls than in controls. After adjusting for age, pubertal stages, and height percentile, MEHHP\% was positively associated with waist circumference and MEOHP\% was negatively associated with body mass index (BMI) percentile. Concentrations of MiBP, MnBP, MEHP, MEHHP, sum of DEHP metabolites, and sum of high molecular weight phthalates (HMP) were positively associated with serum ALT. Concentrations of MiBP were also positively associated with total cholesterol/LDL-cholesterol levels. After controlling for age and pubertal stages, MEHHP\% was positively associated with fasting insulin and HOMA-IR, whereas MEOHP\% was negatively associated with fasting insulin and HOMA-IR. However, after further adjustment for BMI percentile, the significant associations were remained only for MEOHP\%.
\end{abstract}

Conclusions: Urinary concentrations of several phthalate metabolites were positively associated with serum ALT levels, and $\mathrm{MEOHP} \%$ was negatively associated with insulin resistance. Prospective studies are needed to determine potential causal links between phthalate exposure and metabolic derangement in children.

\section{Bariatric Surgery in Obese Adolescents: A Meta-Analysis}

\author{
Katie Feeney", Tania B. Huedo-Medina and Ali Corso \\ Synthesis of Individual Participant Evidence Data (SIPED) Research Lab, University of Connecticut, CT, USA
}

\begin{abstract}
Objectives: The meta-analysis was conducted to synthesize data on adolescent bariatric surgeries. Analysis was focused to assess the efficacy of weight reduction, comorbidity resolution, quality of life improvements, and the safety of procedures.

Methods: A comprehensive literature search was conducted based on predetermined inclusion criteria. The search returned 21 interventions (18 reports; $\mathrm{N}=625$ patients), composed of 3 LSG interventions, 10 LAGB interventions, and 8 RYGB interventions. Fixed-effect and random-effect models were used to calculate overall effect sizes. Moderators were assessed using meta regression.

Results: Analysis shows significant reductions in weight across surgery types $[\mathrm{d}+=-2.1854(-4.1114$ to -0.2594$)$. Weightrelated comorbidities have considerable resolution rates. Notably, cardiovascular risk factors resolved in $62.06 \%$ of patients [LN $(p)=-0.9691(-1.5056$ to -0.4327$)]$. Significant quality of life improvements was apparent after surgery $[\mathrm{d}+=1.9181(1.6430$ to 2.1771)]. Surgical complications occurred in $19.76 \%$ of patients [LN (p) $=-1.4012(-1.8661$ to -0.9363$)]$. Meta regression of weight shows a positive moderation in $\operatorname{LAGB}[\beta=1.3950(0.5523$ to 2.2376$)]$ and a negative moderation in $\mathrm{RYGB}[\beta=$
\end{abstract}


$-1.5823(-2.9269$ to -0.2376$)]$.

Conclusions: Overall, the current literature supports bariatric surgery in adolescents. Further comparative research of surgeries should be conducted to balance beneficial outcomes with procedure safety.

\title{
Efficacy of "Sacbe", a Clinical Education Program, to Decrease Glucose Abnormal Fasting in Mexican Children/Adolescents with Overweight/Obesity and Relatives with Type 2 Diabetes
}

\author{
Ana Rodriguez-Ventura", Bernarda Sánchez, Reyna Sámano, Arturo Parra, Luis Monroy, Florinda Palacios and Maricruz \\ Tolentino
}

Instituto Nacional de Perinatologia, Mexico City, Mexico

\begin{abstract}
Introduction: Before the global epidemic of overweight/obesity is important to identify adolescents with risk factors to develop type 2 diabetes and to reverse pre-diabetic states through of integral clinical education programs.

Methods: Sacbe, a Mayan word that means "the way", is a clinical education program based of a healthy lifestyle (DPP, TODAY) sessions of groups, active participation of parents and an integrated team of nutritionists, nurses and physicians inside a socio cultural context previously analyzed and already published. Inclusion criteria were: any gender, age between 9 and 20 years, overweight or obesity ( $\geq$ body mass index -BMI- percentile 85 , according CDC tables), relatives with diabetes, and being Mexican.
\end{abstract}

Results: Fifty-five children and 65 parents participated, $63 \%$ was female sex, media age was $14.3 \pm 3$ years, $35 \%$ presented overweight (OW) and $65 \%$ obesity (OB), after the first 3 months, OB decreased until $42.5 \%, 3 \%$ achieved a normal BMI; $\mathrm{BMI}$ and weight decreased significantly $(\mathrm{p}=0.001)$. The $29 \%$ presented glucose abnormal fasting $(\mathrm{GAF})$ and $9 \%$ intolerance glucose; 43\% hyperinsulinism ( $\geq 15), 67.5 \%$ HOMA $\geq 2.5,66 \%$ HbA1c between 5.7-6.4\%, 11.5\% hypercholesterolemia and $31.5 \%$ hypertriglyceridemia. Anyone had diabetes. After 3 months of intervention, GAF decreased until $9 \%(\mathrm{p}<0.02)$ and hyperinsulinism until 32.4\% ( $\mathrm{p}<0.000)$.

Conclusions: An integral intervention inside a socio cultural context is effective to decrease $\mathrm{OW}$ and $\mathrm{OB}$ and to reverse GAF and hyperinsulinism in children/adolescents with other risks to develop type 2 diabetes (racial group and relatives with diabetes).

\section{Long-term Supplementation of a Functional Sugar with Iso-caloric Diet Improved the Glycemic Control with Weight Loss in Diet-induced Obese Mice}

\author{
Youngji Han ${ }^{1,2^{*}}$, Ga Young Do ${ }^{1,2}$, Yun Jin Kim ${ }^{1,2}$ and Myung-Sook Choi ${ }^{1,2}$ \\ ${ }^{1}$ Center for Food and Nutritional Genomics Research, South Korea \\ ${ }^{2}$ Department of Food Science and Nutrition, Kyungpook National University, South Korea
}

\begin{abstract}
Recently there has been a global shift in diet towards increased intake of energy-dense foods that are high sugars. D-psicose, a C-3 epimer of D-fructose, has been reported as one of anti-diabetic food components; however, its mechanism is not yet completely understood. In present study we investigated in C57BL/6J mice fed high-fat diet using the pair-feeding method. Sixty C57BL/6J mice were divided into six dietary groups and fed a normal diet (ND), a high-fat diet (HFD) (20\% fat, $1 \%$ cholesterol, w/w), HFD with D-psicose (PSI) supplement for 16 weeks. In order to investigate mechanism, we analyzed biochemical changes to compare the physiological effect of other sugar substitutes. In our study, body weights and fasting blood glucose levels were significantly increased in HFD group compared to ND group. Also glucose-dependent insulin tropic polypeptide (GIP), leptin, resistin and adiponectin levels and leptin: adiponectin ratio in ND-fed mice was drastically higher than HFD-fed mice. However long-term consumption of D-psicose in HFD-fed mice drastically reduced not only body weight and fasting glucose levels also concentration of hormone and adipokines that are related to insulin resistance compared to HFD groups. Moreover, supplementation of D-psicose drastically reduced the hepatic glucose-regulating enzyme activities such as glucokinase, PEPCK and glucose-6-phosphate compared to HFD groups. Thus D-psicose supplement with Iso-caloric highfat diet improves the glycemic control via regulation of hepatic glucose-regulating enzyme activities and reduced adipokines levels which is related to insulin resistance.
\end{abstract}




\title{
Insulin Resistance in Spontaneously Obese Rhesus Monkeys (Macaca mulatta)
}

\author{
Charon de Villiers ${ }^{*}$, Joritha van Heerden ${ }^{2}$ and Johan Louw ${ }^{3}$ \\ ${ }^{1}$ Delft Animal Centre, South African Medical Research, Council, Cape Town, South Africa \\ ${ }^{2}$ Primate Unit, South African Medical Research Council, Cape Town, South Africa \\ ${ }^{3}$ Biomedical Research and Innovation Platform, South African Medical Research Council, Cape Town, South Africa
}

\begin{abstract}
Introduction: Spontaneous obesity and diabetes have been observed in a variety of non-human primate species including rhesus, cynomologus, vervet monkeys and baboons with deposition of excessive fat that is most noticeable in the abdominal region. As in humans, nonhuman primates have a period of obesity-associated insulin resistance and hyperinsulinaemia that is initially met with compensatory insulin secretion. This period is followed by continued deterioration of insulin secretory capacity and with continued insulin resistance and further pancreatic reserves; there is generally a sharp increase in glucose that prompts treatment to prevent ketosis and acidosis. As in humans, the majority of cases reported in nonhuman primates represents Type 2 diabetes (T2D) and are associated with both obesity and increasing age.
\end{abstract}

Aim: We report on nine adult rhesus female monkeys that developed spontaneous obesity with increased bodyweight and waist circumference.

Methods:

- Measurements of bodyweight, length and waist circumference

- Measurements of crown-rump length

- Circumference of the abdomen

- Nine obese monkeys and nine control monkeys were starved overnight before the intravenous glucose tolerant test (IV-GTT)

- A polyethylene catheter was used for infusion of glucose ( $1 \mathrm{ml}$ of $50 \%$ glucose $/ \mathrm{kg}$ bodyweight over one minute) and for blood collection

- Samples were collected at baseline, 5, 10, 15, 30, 45 and 60 minutes

Results:

- High bodyweights were primarily attributed to body fat rather than to statues or lean body mass

- Abdominal circumference and abdominal adipose fat were highly correlated with total body fat

- Compared to the controls, the obese individuals had a similar glucose uptake

- Major differences were obtained in insulin synthesis with obese individuals

- It is clear that these obese individuals are in a pre-diabetic state

Conclusion: The investigation of South African traditional medicines that have the potential to alleviate obese related diseases, are of interest. The rhesus model can be useful for testing novel substances.

\section{Correlation Between the Glycemic Variability with the Circadian Variability of Blood Pressure in Individuals with Normal Weight and Normal Glucose Tolerance}

\author{
Lizet Rosales-Rivera, Laura-Yareni Zuñiga, Luis Hernández-Palma, Diego Hernández-Molina, Ernesto Cardona- \\ Muñoz, Carlos Ramos-Becerra, Julia Ramos-Núñez, Manuel González-Ortiz and Esperanza Martínez-Abundis
}

Institute of Experimental and Glinical Therapeutics, University of Guadalajara, Guadalajara, Mexico

\begin{abstract}
Glycemic variability and variability in blood pressure have been associated with the development and progression of cardiovascular risk; there is not information of the correlation between the glycemic variability with the circadian variability of blood pressure in individuals with normal weight and normal glucose tolerance.

A cross-sectional study was carried out in 11 subjects of 30 to 40 years, with a body mass index between $18.5-24.9 \mathrm{~kg} / \mathrm{m}^{2}$, with normal glucose tolerance. Each individual had a clinical and laboratory evaluation (glucose, uric acid, creatinine, lipid profile and transaminases). An ambulatory continuous glucose monitoring was performed each 5 min for $72 \mathrm{~h}$ using an iPro2 (Medtronic, Northridge, CA) and a $24 \mathrm{~h}$ ambulatory blood pressure monitoring every $15 \mathrm{~min}$ (Microlife WatchBP, Switzerland).
\end{abstract}


Mean amplitude of glycemic excursions (MAGE), area under the curve of glucose, mean of daily differences, ratio of blood pressure variability, linear regression and Pearson correlation coefficient were calculated. A p $\leq 0.05$ was considered significant.

The group average MAGE was $22.79 \pm 7.25 \mathrm{mg} / \mathrm{dl}$. The variability in systolic blood pressure $7.73 \pm 1.60 \mathrm{mmHg}$ and in diastolic blood pressure $10.73 \pm 2.10 \mathrm{mmHg}$. A significant negative correlation was found between MAGE with the ratio of variability of systolic blood pressure $(r=-0.726, p<0.05)$ and the ratio of variability of diastolic blood pressure $(r=-0.924, p<0.05)$.

The MAGE and the circadian variability of blood pressure are within the expected for subjects with normal weight, without impaired glucose tolerance. There was a negative correlation between glucose and blood pressure and between the glycemic variability and the circadian variability of blood pressure.

\title{
Momordica charantia Reduces Body Weight, Fat Percentage and Waist Circumference in Type 2 Diabetes Mellitus Patients
}

\author{
Marisol Cortez-Navarrete*, Esperanza Martinez-Abundis, Karina Griselda Perez-Rubio and Manuel Gonzalez-Ortiz \\ Institute of Experimental and Clinical Therapeutics, Department of Physiology, Health Sciences University Center, University of \\ Guadalajara, Mexico
}

\begin{abstract}
Momordica charantia is a popular plant used for the treatment of diabetes. Several clinical and animal models studies have demonstrated favorable results in metabolic and cardiovascular profiles.

The aim of this study was to evaluate the effect of Momordica charantia administration in metabolic control parameters in patients with type 2 diabetes mellitus (T2DM).

A randomized, double blind, placebo controlled clinical trial was carried out in 24 adults with newly diagnosed T2DM. Patients received Momordica charantia (1000 mg orally, twice daily) or placebo for 3 months. An oral glucose tolerance test (OGTT) of 2-h was done before and after the intervention. Glycated hemoglobin (A1C), blood pressure, weight, body mass index (BMI), waist circumference (WC) and fat \% were evaluated. Statistical analyses were performed with Wilcoxon and Mann-Whitney U tests. An Ethic Committee approved the protocol and a written informed consent was obtained from all volunteers.

In the Momordica charantia group, there were significant decreases in weight $(79.4 \pm 9.2 \mathrm{vs} .78 .0 \pm 9.2 \mathrm{~kg}, \mathrm{p}=0.007)$, BMI $\left(29.1 \pm 2.4\right.$ vs. $\left.28.3 \pm 1.9 \mathrm{~kg} / \mathrm{m}^{2}, \mathrm{p}=0.007\right)$, fat $\%(36.7 \pm 7.8$ vs. $36.3 \pm 7.6 \%, \mathrm{p}=0.021)$, WC $(106 \pm 12$ vs. $104 \pm 11 \mathrm{~cm}$, $\mathrm{p}=0.013), \mathrm{A} 1 \mathrm{C}(7.8 \pm 0.8$ vs. $7.1 \pm 1.3 \%, \mathrm{p}=0.039)$ and $2-\mathrm{h}$ glucose in an oral glucose tolerance test $(\mathrm{OGTT})(17.1 \pm 3.7 \mathrm{vs}$. $13.2 \pm 4.3 \mathrm{mmol} / \mathrm{l}, \mathrm{p}=0.008)$. The placebo group showed no changes after the intervention.
\end{abstract}

In conclusion, Momordica charantia reduced A1C, 2-h glucose, weight, BMI, fat \% and WC.

\section{A Study on Obesity Inside the Prison System}

\section{Flavia Auler ${ }^{*}$, Gilmara Braz de Lima ${ }^{1}$, Marcelly Caroline Pires Fernandes ${ }^{1}$, Larissa Dantas Guidoti ${ }^{1}$, Wesley Marcos de Almeida $^{2}$ and Evandro Henrique Cavalheri ${ }^{2}$}

${ }^{1}$ Nutrition Course, Pontifical Catholic University of Paraná, Curitiba, PR, Brazil

${ }^{2}$ Statistical responsible, Pontifical Catholic University of Paraná, Curitiba, PR, Brazil

\begin{abstract}
The increase in the female prison population is a sad reality in Brazil, between 2000 and 2014 the increase was 567\% while only $220 \%$ was found in men. Because little research on the health profile in incarcerated women, the objective of this study was the health profile of chronic diseases contemplating social aspects, risk factors, care and health perception relating them to obesity. Descriptive cross-sectional study with a quantitative approach, performed in the Female Penitentiary of Paraná, with 10 diabetic and 41 hypertensive patients with a positive record and the data were analyzed with SPSS. Most were of low economic class (54.9\%), ex-smokers (68.6\%) and sedentary (68.6\%). Regarding health care, the average time of the last doctor appointment was 6.1 months, $76.5 \%$ had never performed the measurement of waist circumference. The BMI average was 26.1 $+3.8 \mathrm{~kg} / \mathrm{m}^{2}$ with a high prevalence of obesity (66.6\%) and abdominal obesity (72.5\%), being the only variable with statically significance (OR $3.2 \mathrm{p}<0.05$ ) with obesity. It was observed that $60 \%$ of women gain weight during incarceration $(0.4 \mathrm{~kg} /$ month). Obesity did not influence the glycemic control, but the average blood pressure values indicate uncontrolled (177 x 132
\end{abstract}


$\mathrm{mmHg}$ ), despite the perception of control show $47.9 \%$. From these results, it was found that significant weight gain during incarceration and may interfere with the control of hypertension. There is a concern of local health staff in the control of diabetes and hypertension, but there is no effective monitoring of nutritional parameters and these could prevent the complications of these diseases.

\title{
Health Conditions of Recyclable Waste Pickers
}

\author{
Flavia Auler ${ }^{*}$, Roberto K. N. Cumang and Alika T. A. Nakashima ${ }^{3}$ \\ ${ }^{1}$ Pontifical Catholic University of Paraná, Curitiba, PR, Brazil \\ ${ }^{2}$ State University of Maringá, Maringá, $P R$, Brazil \\ ${ }^{3}$ Pontifical Catbolic University of Paraná, Curitiba, PR, Brazil
}

\begin{abstract}
The presence of chronic non-communicable diseases (NCDs) among vulnerable populations is a rarely discussed subject. Thus, the present cross-sectional descriptive study aimed to analyze the health conditions and access to public health services of 268 waste pickers in southern Brazil. Regarding NCDs, their prevalence of hypertension was 32.8\%, diabetes 11.4\%, dyslipidemia $16.4 \%$, overweight $51.1 \%$, obesity $25.7 \%$ and abdominal obesity $57.8 \%$. Regarding access to health services, $36.9 \%$ of the sample had not consulted with a doctor in more than one year, $15.7 \%$ had no access to a healthcare unit, $7.5 \%$ had never measured their blood pressure, $45.9 \%$ had never had a glucose test, and $61.2 \%$ had never had a triglyceride test. The statistical analysis showed that whereas men had gone longer since their last medical consultation and blood pressure measurement, women had increased frequencies of overweight, abdominal obesity, awareness of their hypertension and use of anti-hypertensive drugs ( $<<0.05)$. Therefore, the health profile of waste pickers is critical, with a high prevalence of NCDs and poor healthcare usage. Strategies to improve monitoring and treatment are recommended.
\end{abstract}

\section{Simulation of an in vitro NAFLD Model Showed Inhibition of Lipid Accumulation and Lipotoxicity by Picroside II}

\author{
Hiteshi Dhami-Shah ${ }^{1-3^{*}}$, Rama Vaidya ${ }^{1}$, Shobha Udipi ${ }^{1,3}$, Srividhya Raghavan ${ }^{2}$, Shiny Abhijit ${ }^{2}$, Viswanathan Mohan ${ }^{2}$, \\ Muthuswamy Balasubramanyam ${ }^{2}$ and Ashok Vaidya ${ }^{1}$
}

${ }^{1}$ Medical Research Centre of Kasturba Health Society, Mumbai, India

${ }^{2}$ Madras Diabetes Research Foundation, Chennai, India

${ }^{3}$ S.N.D.T University, Mumbai, India

\begin{abstract}
In South Asians, Non-Alcoholic Fatty Liver Disease (NAFLD) patients with normal BMI are often “metabolically obese”. In addition, high proclivity of Indians for visceral obesity increases their likelihood for NAFLD with a subsequent progress into aggressive stages like steatohepatitis, cirrhosis \& hepatic carcinoma. Despite high prevalence (men: $35.1 \%$, women: $29.1 \%$ ) of NAFLD in India, its identification \& management remains neglected. Liver-directed pharmacotherapy may form an important component of the comprehensive management of NAFLD. Our team has shown the efficacy of Picrorhiza kurroa in viral hepatitis using double blind placebo control trial. The hepatoprotective activity of Picrorhiza kurroa was further shown in a high fat diet (30\%), male Wistar rat model of NAFLD. Extracts of roots \& rhizomes of Picrorhiza kurroa showed a significant dosedependent reduction in lipid content with normal liver histology compared to Silymarin. However, its effect against NAFLD needs to be explored both mechanistically as well as mode of action point of view in appropriate cell-models. Therefore, we simulated \& standardized an in-vitro model of fatty infiltration in hepatocytes (HepG2 cells) with the experimental induction of lipotoxicity so as to have a rapid drug-screening platform. Cells treated with Picroside-II of Picrorhiza Kurroa \& Silibinin of Silymarin (positive control), have shown changes in the markers of lipogenesis, gluconeogenesis \& $\beta$-oxidation. The mRNA \& protein expression levels of SREBP-1c \& PEPCK were significantly reduced on treatment either with Picroside II or Silibinin $(\mathrm{p}<0.005)$. The observed reduction in TNF- $\alpha$ \& IL-6 levels indicated anti-inflammatory activity of these phytomolecules. As Picroside II appears to be a novel lead molecule against NAFLD, further studies are being underway to investigate its regulatory roles in lipid peroxidation, mitochondrial function, DNA damage \& ATP levels.
\end{abstract}




\title{
Body Mass Index and Chronic Hepatitis in Teenagers in an HCV-Endemic Township in Taiwan
}

\author{
Chia-Yen Dai ${ }^{1-3^{*}}$, Po-Cheng Liang ${ }^{2}$, Chun-Yi Huang ${ }^{2}$, Chung-Feng Huang ${ }^{2,3}$, Ming-Lun Yeh ${ }^{2,3}$, Jee-Fu Huang, ${ }^{2,3}$ Wan- \\ Long Chuang ${ }^{2,3}$ and Ming-Lung $\mathbf{Y u}^{2,3}$ \\ ${ }^{1}$ Health Management Center, Kaobsiung Medical University Hospital, Kaobsiung Medical University, Taiwan \\ ${ }^{2}$ Hepatobiliary Division, Department of Internal Medicine, Kaohsiung Medical University Hospital, Kaohsiung Medical University, \\ Taiwan \\ ${ }^{3}$ Faculty of Internal Medicine, College of Medicine, Kaohsiung Medical University, Taiwan
}

\section{Abstract}

Background and Aims: With a high prevalence of hepatitis $\mathrm{B}$ virus (HBV) and hepatitis $\mathrm{C}$ virus (HCV) infection in Taiwan, the present study aimed to assess the prevalence of $\mathrm{HBV}$ and $\mathrm{HCV}$ infection among teenagers in an $\mathrm{HCV}$ and $\mathrm{HBV}$ endemic township in southern Taiwan.

Methods: We have conducted a cross-sectional survey in the junior higher school located in Tzukuan Township where $\mathrm{HCV}$ and HBV is endemic. 513 teenagers with body mass index (BMI) $21.5 \pm 4.4 \mathrm{~kg} / \mathrm{m}^{2}$ were enrolled and received tests for serum liver enzyme, serum marker of hepatitis B and C, and serum HBV DNA or HCV RNA by real-time PCR assay if need.

Results: The servo prevalence of $\mathrm{HBsAg}$, anti-HBs, anti-HBc, and anti-HCV were $2.1 \%, 2.5 \%, 20.3 \%$, and $0.6 \%$ respectively. 45.5\% of the $11 \mathrm{HBsAg}$ carriers were positive for HBeAg and with high HBV DNA levels more than 7 log IU/mL. $33.3 \%$ of the $6 \mathrm{HBeAg}$-negative subjects have positive HBV DNA (397 and 2,179 IU/mL, respectively). All three subjects with positive anti-HCV had negative HCV RNA. Students with positive HBsAg had significantly lower body weight and BMI than students with negative $\mathrm{HBsAg}(\mathrm{P}=0.031$ and $\mathrm{P}=0.017$, respectively $)$ in univariate analysis. In multivariate analysis, lower $\mathrm{BMI}$ was the only significant factors associated with positive $\mathrm{HBsAg}$ (odds ratio: 0.691, 95\% CI: 0.539-0.885, P = 0.03).

Conclusions: A high prevalence of $\mathrm{HBsAg}$ and anti-HCV and low prevalence of anti-HBs, and $\mathrm{HCV}$ viremia were noted among teenagers in this HCV hyperendemic area. HBV infection is associated with lower body weight and BMI.

\section{Prenatal Metformin Treatment Prevents Estradiol Increase and Partially Improves Ovarian Function in Offspring of Obese Mothers}

\author{
Gonzalo Cruz $^{1 *}$, Daniela Alvarez ${ }^{1}$, Sofia Olguin ${ }^{1}$, Karina Ceballo ${ }^{1}$, Daniela Fernandois ${ }^{1}$, Ramon Sotomayor ${ }^{2}$ and Jonathan \\ Martinez ${ }^{2}$ \\ ${ }^{1}$ Laboratory of Reproductive and Metabolic Disorders, Facultad de Ciencias; Centro de Neurobiologia y Plasticidad Cerebral (CNPC), \\ Instituto de Fisiologia, Universidad de Valparaiso, Valparaiso, Chile \\ ${ }^{2}$ Laboratory of Neurochemistry and Neuropharmacology, Facultad de Ciencias; Centro de Neurobiologia y Plasticidad Cerebral (CNPC), \\ Instituto de Fisiologia, Universidad de Valparaio, Valparaiso, Chile
}

\begin{abstract}
Obesity epidemic is one of the major concerns in the world. Maternal obesity leads to several abnormalities on offspring such as cardiovascular, metabolic and reproductive disorders. We previously demonstrated that maternal obesity in rats leads to liver dysfunction, increased serum estradiol, advanced puberty and ovarian follicular alterations in the progeny. We aimed to determine if metformin prevents this programming produced by a high fat diet exposure. Sprague Dawley rats were distributed in 3 groups: Control diet (13\% Kcal in fat); High Fat Diet (HF) (60\% Kcal in fat, Research Diet, USA) and HF+ Metformin $(60 \% \mathrm{Kcal}$ in fat + metformin $150-200 \mathrm{mg} / \mathrm{Kg}$ in tap water). Diet was administered for 1 month previous to pregnancy, during pregnancy and nursing. Metformin was administered from 1 week previous to pregnancy until weaning of the offspring. Weight gain during pregnancy and offspring weight were not affected by prenatal metformin. At postnatal day (PND) 14 metformin tended to prevent the estradiol increase while at PND60 metformin significantly prevented the estradiol increase. Coherently, hepatic CYP3A2 (enzyme that metabolizes estradiol) decreased in offspring obese mothers and this decrease was prevented by metformin treatment. The generation of ovarian cyst was also prevented by metformin in offspring of obese mothers. In conclusion, metformin prevented some reproductive alterations triggered by maternal obesity on the offspring.
\end{abstract}

Financial Support: Fondecyt grant 11130707 to G.C. 


\title{
Intra Cerebro Ventricular Administration of Leptin Increases Norepinephrine Content in Fat Tissue, Liver and Ovary: Implications on Metabolic and Reproductive Function
}

\author{
Daniela Fernandois ${ }^{1^{*}}$, Gonzalo Cruz ${ }^{1}$, Maria Jesus Vazquez ${ }^{2}$ and Manuel Tena-Sempere ${ }^{2}$ \\ ${ }^{1}$ Laboratory of Reproductive and Metabolic Disorders, Facultad de Ciencias; Centro de Neurobiología y Plasticidad Cerebral (CNPC), \\ Instituto de Fisiologia, Universidad de Valparaiso, Valparaiso, Chile \\ ${ }^{2}$ Department of Cell Biology, Physiology and Immunology, University of Cordoba; CIBER Fisiopatologia de la Obesidad y Nutricion, \\ Instituto de Salud Carlos III; Instituto Maimonides de Investigacion Biomedica de Cordoba/Hospital Universitario Reina Sofia, \\ Cordoba, Spain
}

\begin{abstract}
Obesity is strongly associated with the development of cardiovascular, metabolic and reproductive dysfunctions. On the other hand, obstructive sleep apnea and chronic stress, both causing sympathetic activation, are associated to metabolic and reproductive alterations, including polycystic ovary syndrome (PCOS). In this context, we aimed to study if leptin, which is increased in obese individuals, is mechanistically associated with an increase in the adrenergic tone in the ovary, liver and different types of fat tissue. Groups of female Wistar rats were treated icv with vehicle (Control) or leptin (7.5 $\mu \mathrm{g} / 12-\mathrm{h}$ for 5 days). After completion of the treatments, the rats were sacrificed and different tissues were collected. Norepinephrine was measured by ELISA. Norepinephrine content increased in the ovary, liver, visceral adipose tissue and brown adipose tissue. Notably, norepinephrine in ovary leads to cyst formation in models of ovarian sympathetic hyperactivity. In addition, hepatic norepinephrine is also related to liver insulin resistance and non-alcoholic fatty liver disease (NAFLD), while the increase in norepinephrine in the adipose tissue is related to elevated levels of free fatty acids, which might contribute to hepatic insulin resistance. We propose that the increase in leptin during obesity activates the sympathetic nervous system output to the ovary, liver and adipose tissue. Thereby, increased norepinephrine could participate in the pathogenesis of common disorders, such as NAFLD and PCOS, linked to obesity.
\end{abstract}

Financial Support: Fondecyt grant 11130707 to G.C. and BFU2014-57581 grant to M.T-S.

\section{High Free Fatty Acids Induced Aberrant Activation of TGF- $\beta$ Signal: Link Between Obesity and Breast Cancer Prognosis}

\author{
Yong Wu ${ }^{1,2^{*}}$, Sami Dwabe ${ }^{1}$, Mohammad Atefi ${ }^{1}$, Yahya Elshimali ${ }^{1}$, Kevin T. Kemp II $^{1}$, Jesse Haro ${ }^{1}$, Marianna Sarkissyan ${ }^{1}$, \\ Jay Vadgama ${ }^{1,2}$, Xiaoting $\mathrm{Yu}^{3}$ and Xianghua $\mathrm{Yi}^{3}$ \\ ${ }^{1}$ Division of Cancer Research and Training, Department of Internal Medicine, Charles R. Drew University of Medicine and Science, \\ Los Angeles, CA, USA \\ ${ }^{2}$ David Geffen School of Medicine, University of California, Los Angeles, CA, USA \\ ${ }^{3}$ Department of Pathology, Tongji Hospital, Tongji University School of Medicine, Shanghai, China
}

\begin{abstract}
Obesity is an independent risk factor for breast cancer. It has also been associated with poorer prognosis, increased incidence, and a more aggressive tumor phenotype. The mechanisms behind this correlation are poorly defined. Most obese individuals have high plasma free fatty acids (FFA) levels. Increasing evidence points to FFA signaling playing an important role in tumorigenesis and breast cancer development and progression. In spite of the physiological significance of FFA in the breast cancer biology, the precise molecular mechanisms by which FFA might influence cancer development and progression still remain to be elucidated. Here, we demonstrated that TGF- $\beta$ transiently activates the extracellular signal-regulated kinase (ERK) and consequently phosphorylates SMAD4 at Threonine 277, which promotes SMAD4-USP9x interaction, SMAD4 nuclear retention, SMAD3-SMAD4 complex nuclear accumulation, and stimulated TGF- $\beta$ target gene Twist transcription in human breast cancer cells. Inhibition of ERK with its specific inhibitor leads to SMAD4 de-phosphorylation and USP9xSMAD4 disassociation, resulting in SMAD4 nuclear export and termination of Twist transcription. Furthermore, loss of USP9 $x$ eliminates TGF- $\beta$-induced SMAD4 nuclear accumulation and SMAD3/SMAD4 formation, leading to repression of Twist expression. In addition, FFA further promotes TGF- $\beta$-induced ERK activation, SMAD4 phosphorylation, SMAD4 nuclear accumulation and SMAD3/SMAD4 formation, thus facilitating TGF- $\beta$-dependent cancer progression. Importantly, suppression of ERK and USP9x inhibits obesity-induced metastasis in vivo. Together, our study highlights the dynamic interaction of USP9 $x$ and SMAD4 for regulating TGF- $\beta$ signaling and dyslipidemia-induced aberrant TGF- $\beta$ activation during breast cancer metastasis.
\end{abstract}




\title{
The Effects of Diet Containing Low and High Levels of the Soy Isoflavone Daidzein on Liver Steatosis in Obese Female Zucker Rats
}

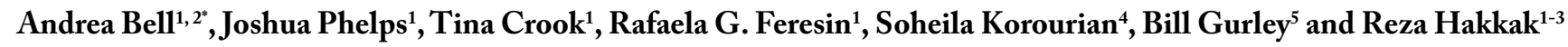 \\ ${ }^{1}$ Department of Dietetics and Nutrition, University of Arkansas for Medical Sciences (UAMS), AR, USA \\ ${ }^{2}$ Arkansas Children's Hospital Research Institute, Little Rock, AR, USA \\ ${ }^{3}$ Department of Pediatrics, UAMS, AR, USA \\ ${ }^{4}$ Department of Pathology, UAMS, AR, USA \\ ${ }^{5}$ Department of Pharmaceutical Sciences, UAMS, AR, USA
}

\begin{abstract}
Obesity increases the risk for chronic diseases including non-alcoholic fatty liver disease (NAFLD). Hepatic steatosis initiates NAFLD, and progression of NAFLD can result in steatohepatitis, fibrosis and liver failure. Previously, we reported a diet containing high-isoflavone soy protein isolate (HISPI) compared to casein was associated with significantly lower liver weights and liver steatosis scores in obese Zucker rats (OZR). The objective of this study was to investigate whether daidzein, a soy isoflavone in HISPI, is the component of HISPI responsible for reduced liver steatosis. We hypothesized a casein diet containing high-daidzein (HD) compared to low-daidzein (LD) would mitigate hepatic steatosis in female OZR. After one week of acclimation on an AIN-93G diet, 19 five-week-old female OZR (fa/fa) rats were randomly assigned to a modified AIN-93G diet containing either HD $(0.121 \mathrm{~g} / \mathrm{kg}$ feed) or LD $(0.01 \mathrm{~g} / \mathrm{kg}$ feed $)$ ad libitum for 8 weeks. At the end of the experiment, livers were weighed and two 3-mm sections of liver lobe were fixed in $10 \%$ buffered formalin to evaluate for microand macrovesicular steatosis. Steatosis was semiquantitated as a score of 1 to 4 based upon the relative degree of steatosis within hepatocytes: 1) < 25\%, 2) $25-50 \%$, 3) $50-75 \%$, and 4) > 75\%. Our results showed the mean liver weight for HD (n = 10) was $7.05 \%$ of body weight and LD ( $\mathrm{n}=9$ ) was $6.63 \%$ of body weight. The mean liver steatosis scores for HD and LD groups were 3.9 and 3.8 respectively. Differences between mean liver weights $(p=0.278)$ and mean liver steatosis scores $(p=0.62)$ were not significant. In conclusion, daidzein may not be the main isoflavone in HISPI responsible for reducing liver steatosis in OZR. Further research is needed to investigate the other components and mechanisms of HISPI responsible for mitigating hepatic steatosis in OZR.
\end{abstract}

\section{Ursolic Acid Improves Obesity in Metabolic Syndrome}

\author{
Alejandra M. Ramirez-Rodriguez, Manuel Gonzalez-Ortiz, Esperanza Martinez-Abundis and Natalhie Acuna-Ortega \\ Institute of Experimental Therapeutic and Clinical, Mexico
}

\begin{abstract}
Introduction: The metabolic syndrome (MS) is a disease with high prevalence which is characterized by the presence of obesity and increases the risk of developing a cardiovascular disease or type 2 diabetes mellitus. Ursolic acid (UA) is a promising compound for the treatment of obesity in MS since it has demonstrated significant positive effects on metabolism in vitro and in vivo.
\end{abstract}

Aim: To assess the effect of ursolic acid on obesity in patients with metabolic syndrome.

Methods: Randomized, double-blind, placebo-controlled clinical trial in 24 patients with MS without drug treatment. They were randomized to receive $150 \mathrm{mg}$ of UA or placebo, once a day for 90 days. We assessed components of the MS, body weight, BMI and waist circumference. The statistical analysis was evaluated with Wilcoxon and Mann Whitney U tests. An Ethics Committee approved the protocol and written informed consent.

Results: After UA administration, remission of the SM in 50\% of patients compared with the placebo group control was observed $(\mathrm{p}=0.014)$. There was a significant decrease in body weight $(75.7 \pm 11.5 \mathrm{vs} .70 .0 \pm 17.8 \mathrm{~kg}, \mathrm{p}=0.001), \mathrm{BMI}(29.9 \pm$ 3.7 vs. $\left.27.3 \pm 6.4 \mathrm{~kg} / \mathrm{m}^{2}, \mathrm{p}=0.001\right)$, waist circumference $(95.2 \pm 9.4$ vs. $86.6 \pm 10.0 \mathrm{~cm}, \mathrm{p}=0.001)$ and fasting glucose $(6.0 \pm$ 0.5 vs. $4.7 \pm 0.4 \mathrm{mmol} / \mathrm{L}, \mathrm{p}=0.002)$.

Conclusion: UA administration during 90 days achieved remission of MS and significantly decreased body weight, BMI, waist circumference and fasting glucose concentrations. 


\title{
Brown Adipose Tissue Denervation Unexpectantly Enhances Leptin-Mediated Weight Loss
}

\author{
Philip J. Scarpace ${ }^{1^{*}}$, Michael M. Matheny ${ }^{1}$ and Nihal Tumer ${ }^{1,2}$ \\ ${ }^{1}$ Department of Pharmacology and Experimental Therapeutics, University of Florida, Gainesville, FL, USA \\ ${ }^{2}$ Department of Veterans Affairs Medical Center, Gainesville, FL, USA
}

\begin{abstract}
Leptin efficaciously reduces food consumption and body weight (BW) for a transient period, at least in lean rodents. Leptin is also a potent activator of brown adipose tissue (BAT) thermogenesis through induction and activation of uncoupling protein 1 (UCP1). It has long been assumed that increased BAT thermogenesis is one mechanism underlying the leptinmediated decreases in BW. We tested this hypothesis using a rat model in which the interscapular BAT was bilaterally surgically denervated. We previously demonstrated that this procedure prevents the induction/activation of UCP1 by centrally administered leptin. The present study consisted of three groups of F344XBN rats, bilaterally denervated administered vehicle (Den-Control), denervated administered central leptin (Den-Leptin) and sham operated administered leptin (Sham-Leptin). Leptin was delivered via gene delivery of recombinant adeno-associated viral leptin (rAAV-leptin) into the third ventricle. This method provides constant central activation by leptin for up to two years. Delivery of rAAV-leptin to Sham-Leptin decreased BW and FI in the expected manner over 30 days. The other two groups were pair-fed to the Sham-Leptin group. BW in DenControl paralleled the Sham-Leptin indicating the BW reduction in Sham-Leptin was entirely due to the decreased food intake. Surprisingly, BW reduction in the Den-Leptin was greater than either of the other two groups. These data suggest that BAT thermogenesis does not contribute to the leptin-mediated BW reduction in rats, and more importantly, that BAT denervation triggers a compensatory pathway that promotes weight loss, possibly by enhancing leptin action. Discovery of this pathway has the potential to enhance leptin action in the obese state where the effectiveness of leptin therapy is absent.
\end{abstract}

This work has been Supported by NIH DK091710.

\section{Effectiveness of Dietary Fiber Adequacy on Adiposity Reduction in Free-Living Adults under Physical Activity Program}

\author{
Patricia Baston Frenhani", Hugo Tadashi Kano, Fernanda Maria Manzini Ramos, Mariana Santoro Nakagaki and Roberto \\ Carlos Burini
}

Nutrition and Physical Exercise Metabolism Center, Botucatu Medical School-UNESP, Brazil

\begin{abstract}
Dietary fiber adequacy is the key goal as limiting energy consumption when fat loss is the objective. Aiming to reduce obesity by introducing dietary fiber adequacy in a short-term $(10 \mathrm{wk})$ lifestyle modification with physical exercises we selected 50 subjects $(50.0 \pm 11.8$ years old) participants of the epidemiological program for healthy lifestyle promotion ("Mexa-se PróSaúde”). They were assigned in two dietary groups receiving either a regularly counseled diet (G1, n = 22, 5M and $17 \mathrm{~F}, \mathrm{BMI}$ $\left.=32.1 \pm 5.3 \mathrm{~kg} / \mathrm{m}^{2}, \mathrm{WC}=103.2 \pm 12.7 \mathrm{~cm}\right)$ or a supervised intervention with grains, fruits and vegetables for a $30 \mathrm{~g}$ fiber/day adequacy $\left(\mathrm{G} 2, \mathrm{n}=28,6 \mathrm{M}\right.$ and $\left.22 \mathrm{~F}, \mathrm{BMI}=33.6 \pm 5.3 \mathrm{~kg} / \mathrm{m}^{2}, \mathrm{WC}=106.7 \pm 14.4 \mathrm{~cm}\right)$. Both groups were in a supervised aerobic mixed physical exercise during 10 weeks. Anthropometric, dietary and physical fitness assessments were undertaken at baseline and end of experiment. Groups and moments were statistically compared for $p=0.05$. At baseline both groups were similar for all variables. G1 responded to the intervention by keeping the baseline anthropometry. When compared to G1 the G2 showed significantly greater anthropometric reductions. G2 increased the fiber intake by $211 \%$ and plasma $\beta$ carotene by $150 \%$ along with a reduction of body fat (4.0\%), WC (7\%) and severe obesity (16\%). The obesity reduction resulted by the migration of grade III to II (2\%) and II to I (14\%) without changes on overweight and eutrophy. Thus, by adequating dietary fiber improved the quality of the diet and accelerated the body fat loss in obese free-living adults.
\end{abstract}

This work has been supported by CNPq and CAPES. 


\title{
Effects of 8 Weeks of Alternate Day Fasting and Combined Exercise Program on Body Composition, Insulin Resistance, and Blood Lipids in Overweight and Obese Adults: A Pilot Randomized Controlled Study
}

\author{
Ji Won Lee ${ }^{1^{*}}$, Ki Deok Park ${ }^{2}$, Minsuk $\mathrm{Oh}^{3}$ and Ki-yong $\mathrm{An}^{3}$ \\ ${ }^{1}$ Department of Family Medicine, Yonsei University, College of Medicine, Seodaemun-gu, Seoul, Republic of Korea \\ ${ }^{2}$ Department of Rehabilitation Medicine, Gachon University, Gil Medical Center, Incheon, Republic of Korea \\ ${ }^{3}$ Department of Sport and Leisure Studies, Sport Medicine Laboratory, Yonsei University, Seoul, Republic of Korea
}

\begin{abstract}
The purpose of this study is to investigate the effects of ADF and exercise, and combined effect of exercise and ADF on body weight, composition, blood lipids and insulin resistance in overweight or obese adult. Fifty overweight or obese adults were randomized into 4 groups (combination: exercise + ADF, ADF only, exercise only, and control) for 8 weeks. Finally, 35 participants were completed the trial after 8 weeks of intervention. Compared to the control group, the combination group showed greater change in fat mass, and BMI ( $p<0.05)$. Fasting insulin level $(9.4 \pm 3.5 \rightarrow 6.5 \pm 3.0 \mu \mathrm{IU} / \mathrm{ml}, \mathrm{p}<0.05)$, and insulin resistance $(2.4 \pm 1.4 \rightarrow 1.4 \pm 0.8, \mathrm{p}<0.05)$ presented by homeostasis model assessment (HOMA-IR) index were decreased only in the combination group. Only in the combination group, level of triglyceride (TG) reduced. $(133.4 \pm 47.1 \rightarrow 89.7 \pm 33.6 \mathrm{mg} /$ $\mathrm{d}$ l, $\mathrm{p}<0.01)$. High-density lipoprotein cholesterol (HDL-C) was increased after the intervention in the combination (48.0 \pm $10.4 \rightarrow 53.3 \pm 10.5 \mathrm{mg} / \mathrm{dl}, \mathrm{p}<0.05)$, exercise $(44.8 \pm 9.9 \rightarrow 51.5 \pm 8.6 \mathrm{mg} / \mathrm{dl}, \mathrm{p}<0.05)$, and in the control group (54.6 \pm 11.8 $\rightarrow 59.4 \pm 12.4 \mathrm{mg} / \mathrm{dl}, \mathrm{p}<0.05$ ). The 8 weeks of combination intervention (ADF + exercise) is the most effective way to reduce body weight and fat mass, and it reduces TG and glucose level in overweight or obese adults.
\end{abstract}

Acknowledgements: This study was supported by a faculty research grant of Yonsei University College of Medicine for 2013(6-2013-0021). This research was supported by the Bio \& Medical Technology Development Program of the National Research Foundation (NRF) funded by the Ministry of Science, ICT \& Future Planning, Republic of Korea (NRF2013M3A9B6046416).

\section{Evaluation of the Effect of Moderate Intensity Physical Activity on Glycemic Variability in Sedentary Individuals with Normal Weight without Alterations in the Oral Glucose Tolerance}

\author{
Laura-Yareni Zuniga*, Esperanza Martinez-Abundis and Manuel Gonzalez-Ortiz \\ Institute of Experimental and Clinical Therapeutics, University of Guadalajara, Guadalajara, Mexico
}

\section{Abstract}

Background: Currently, it is recognized that physical activity has influence on plasma glucose concentrations. However, so far not known its effect on glycemic variability.

Methods: An open interventional study was carried out in 11 sedentary subjects of 30 to 40 years, with body mass index of $18.5-24.9 \mathrm{~kg} / \mathrm{m}^{2}$ and with normal oral glucose tolerance. After subjects agreed to participate through a signed informed and written consent, was performed an ambulatory continuous glucose monitoring each 5 minutes for 96 hours (4 days), using an iPro2 (Medtronic, Northridge, CA). The first 2 days of the study all the enrolled patients were instructed to continue their usual sedentary lifestyle and the next 2 days they performed 30 minutes of moderate intensity physical activity (60 to $70 \%$ of maximum heart rate). The glycemic variability was calculated by the mean amplitude of glycemic excursions (MAGE), mean of daily differences (MODD) and area under the curve of glucose (AUCG). Statistics analysis: Wilcoxon signed rank test. Statistically significant $\mathrm{p} \leq 0.05$. ClinicalTrials.gov: NCT02620670.

Results: Were enrolled 11 individuals (64\% female and 36\% male). After physical activity were not found significant differences in MAGE (22.92 \pm 6.55 vs. $26.58 \pm 12.18 \mathrm{mg} / \mathrm{dL}, \mathrm{p}=0.168)$, MODD $(12.78 \pm 4.41 \mathrm{vs.} 10.74 \pm 4.34 \mathrm{mg} / \mathrm{dL}, \mathrm{p}=$ $0.168)$ and $\mathrm{ABCG}(27,057 \pm 2,982 \mathrm{vs} .27,037 \pm 2,163 \mathrm{mg} * \mathrm{~h} / \mathrm{L}, \mathrm{p}=0.946)$.

Conclusions: The practice of moderate intensity physical activity (60 to $70 \%$ of maximum heart rate) for 30 minutes by day for 2 days did not modify the glycemic variability in sedentary subjects with normal weight and normal oral glucose tolerance. 


\title{
The Effects of Glycoside from Sea Buckthorns Fruit on Obesity in C56BL/6J Mice Fed High-fat Diet
}

\author{
Youngmi Lee ${ }^{1,2^{*}}$, Jeong Hyeon Lee ${ }^{1,2}$, Ye Jin Kim ${ }^{1,2}$ and Myung-Sook Choi ${ }^{1,2}$ \\ ${ }^{1}$ Center for Food and Nutritional Genomics Research, Korea \\ ${ }^{2}$ Department of Food Science and Nutrition, Kyungpook National University, Korea
}

\begin{abstract}
A high-fat diet which has high energy density results in obesity and, consequently, obesity is caused directly metabolic syndrome such as dyslipidemia, type 2 diabetes and hypertension. Many researchers have attempted to study the effects of a variety of natural materials on metabolic syndrome. The aim of this study was to investigate the effects of glycoside from sea buckthorn fruit on obesity in C57BL/6J mice. Male C57BL/6J mice were divided in 2 groups and were fed high-fat diet $(60 \% \mathrm{kcal}, \mathrm{w} / \mathrm{w})$ and HFD supplemented with GSF (glycoside of sea buckthorn fruit, 0.04\% w/w) for 12 weeks. Body weight was significantly decreased in GSF group compared to HFD group from 5 weeks. Food intake was not different, while food efficiency ratio was significantly lower in GSF group than HFD group. Subcutaneous and visceral white adipose tissue weights were noticeably lower in GSF group compared to HFD group. Whereas, muscle weight was increased in GSF group than HFD group. Free fatty acid level was significantly reduced in GSF group compared to HFD group, although triglyceride and total cholesterol levels were not lowered. Hepatic enzyme activity examination showed that GSF significantly reduced lipogenesisrelated enzymes activities such as malic enzyme (ME), fatty acid synthase (FAS) and phosphatidatephosphohydrolase (PAP). While GSF significantly elevated $\beta$-oxidation activity in GSF group compared to HFD group. Therefore, GSF affected lipid metabolism by decreasing ME, FAS and PAP activities and increasing $\beta$-oxidation. These findings suggest that GSF effective on reducing body weight, improving body composition, and reducing central obesity. Hence, GSF is considered a beneficial natural material for improving adiposity.
\end{abstract}

\section{Lixisenatide vs. Exenatide on Body Weight and Other Parameters of Metabolic Control in Impaired Glucose Tolerance Patients}

\author{
Karina G. Perez-Rubio", Manuel Gonzalez-Ortiz and Esperanza Martinez-Abundis \\ Institute of Experimental and Clinical Therapeutics, Department of Physiology, Health Science University Center, University of \\ Guadalajara, Mexico
}

\section{Abstract}

Currently glucagon-like peptide-1 receptor agonists could be recommended in the treatment of impaired glucose tolerance (IGT) patients. Lixisenatide and exenatide have distinct pharmacokinetic profile and molecular structure with different potential clinical implications.

The aim of this study was to evaluate the effect of lixisenatide vs. exenatide on body weight and other parameters of metabolic control in IGT patients.

A randomized, open-label, clinical trial was carried out in 24 volunteers with IGT. They received SC for 12 weeks lixisenatide (10 $\mu \mathrm{g}$ once daily for 2 week and then $20 \mu \mathrm{g}$ once daily) or exenatide ( $5 \mu \mathrm{g}$ twice daily for 4 weeks and then $10 \mu \mathrm{g}$ twice daily). At the beginning and at the end of the study, body weight, BMI, waist, blood pressure, glucose and lipid profile were measured. Glucose was measured before and after a 75-gram oral dextrose load. Statistical analyses: Wilcoxon and Mann-Whitney U tests. An Ethic Committee approved the protocol and a written informed consent was obtained from all volunteers.

Both groups decreased weight $(-2.0 \pm 2.2$ vs. $-1.8 \pm 1.3 \mathrm{~kg}, \mathrm{p}=0.651)$, BMI, waist, systolic and diastolic blood pressures, as well as, glucose at $120 \mathrm{~min}$. Lixisenatide also decreased fasting glucose $(5.7 \pm 0.8$ vs. $5.0 \pm 0.5 \mathrm{mmol} / 1, \mathrm{p}=0.008)$ and HDL-C $(1.1 \pm 0.1$ vs. $1.0 \pm 0.1 \mathrm{mmol} / 1, \mathrm{p}=0.025)$, and increased LDL-C $(2.5 \pm 0.8$ vs. $3.0 \pm 0.9 \mathrm{mmol} / 1, \mathrm{p}=0.016)$, whereas exenatide decreased triglycerides $(2.4 \pm 1.0$ vs. $2.1 \pm 1.0 \mathrm{mmol} / 1, \mathrm{p}=0.050)$.

In conclusion, both treatments decreased body weight and the same metabolic measurements. Lixisenatide also decreased fasting glucose and HDL-C with increment of LDL-C, and exenatide decreased triglycerides. 


\title{
Supplementation of Ethanol Extract of Platycodon grandiflorum Root Suppress Body Weight Gain and Body Fat Mass in Diet-Induced Obese Mice and Ameliorates Inflammation and Insulin Resistance
}

\author{
Ye Jin Kim ${ }^{1 *}$, Ji-Young Choi ${ }^{2}$, Ri Ryu ${ }^{1}$, Su-Jung Cho ${ }^{1,2}$, Eun-Young Kwon ${ }^{1}$, Myung-Sook Choi ${ }^{1,2}$ and Yong Bok Park ${ }^{3}$ \\ ${ }^{1}$ Department of Food Science and Nutrition, Kyungpook National University Daegu, Republic of Korea \\ ${ }^{2}$ Center for Food and Nutritional Genomics Research, Kyungpook National University, Daegu, Republic of Korea \\ ${ }^{3}$ School of Life Sciences and Biotechnology, Kyungpook National University, Daegu, Republic of Korea
}

\begin{abstract}
Obesity-induced inflammation plays a pathogenic role in development of insulin resistance, and various adipokines are linked to inflammation. Platycodon grandiflorum (PG), one of oriental herbal, is well known to have beneficial effects on obesity. In this study, we demonstrated the metabolic effects of Platycodon grandiflorum root ethanol extract (PGE) on insulin resistance and inflammation in diet induced obesity. C57BL/6J mice (4-week-old, male) were fed a normal diet (16.58\% kcal from fat, ND), high-fat diet (60\% kcal from fat, HFD), and HFD supplemented with 5\% (w/w) PGE. They were given free access to food and distilled water for 12 weeks. In DIO mice, PGE treatment significantly decreased fat mass, body weight as well as plasma level of triglycerides, total-cholesterol, and free fatty acid compared to HFD group. Also, PGE decreased plasma glucose level and insulin resistance index (HOMA-IR) and improved glucose tolerance. PGE led significant decrease in adipokine markers in plasma, such as resistin, PAI-1, IL-6, and TNF $\alpha$ levels. Moreover, PGE improved inflammation by downregulating leptin and TNF $\alpha$ gene expression in epididymal WAT. Together, our findings suggest that PG is one of bioactive material for improving HFD-induced inflammation and insulin resistance by regulating plasma adipokines levels as well as expression of inflammation-related gene.
\end{abstract}

\section{Adequate Fruit Intake and Moderate to High Physical Activity are Protective Factors against Abdominal Obesity in a Brazilian Free-Living Adults}

\author{
Roberto Carlos Burini ${ }^{1 *}$, Hugo Tadashi Kano ${ }^{1}$, Mariana Santoro Nakagaki ${ }^{1}$ and Katia Cristina Portero McLellan ${ }^{2}$ \\ ${ }^{1}$ Botucatu Medical School-UNESP, Brazil \\ ${ }^{2}$ Texas Institute for Kidney and Endocrine Disorders, TX, USA
}

\begin{abstract}
Abdominal obesity is an independent risk factor for cardiovascular diseases, type-2 diabetes mellitus, high blood pressure, dyslipidemias, fibrinolysis and some types of cancer. This study aimed to characterize the major factors associated with abdominal obesity in free-living adults. Baseline data from 1,557 subjects enrolled in an ongoing epidemiological program for healthy lifestyle promotion were used ("Mexa-se Pró-Saúde"). The analyzed data had waist circumference (WC) as primary variable and demographic, socio-economic, anthropometric, clinical, biochemical and dietary food intake data as co-variables. High WC and other metabolic syndrome (MetS) components were defined by NCEP-ATP III (2005). Statistical analysis included comparisons of continuous and categorized variables for $\mathrm{p}=0.05$. The prevalence of high WC was $62.5 \%$. Individuals with high WC consumed mostly a low variety diet rich in fat, predominantly saturated fats and fewer fruit servings. They showed also higher blood pressure and higher plasma levels of glucose, triglyceride, uric acid, CRP, and high HOMA-IR, as well as lower HDL-C values. WC correlated negatively with fruit intake and aerobic capacity (VO2 max). After adjustments by gender, age and energy intake, the $\mathrm{CHO}$ intake $(\mathrm{OR}=1.72, \mathrm{CI}: 1.14-2.58)$ and fruit intake $(\mathrm{OR}=0.68, \mathrm{CI}: 0.49-0.96)$ remained as risk and protecting factors, respectively. Thus, adequate fruit intake and moderate to high levels of physical activity are protecting factors against abdominal obesity whose normalization protects against all the MetS components as well as probably against the pro-inflammatory and oxidative stresses.
\end{abstract}

This work has been supported by CNPq and CAPES. 


\title{
Nrf2 Activation by Glucoraphanin Alleviates Obesity and Insulin Resistance through Increased Energy Expenditure and Alternative Activation of Macrophages
}

\author{
Tsuguhito Ota ${ }^{1^{*}}$, Liang Xu${ }^{1}$, Fen Zhuge ${ }^{1}$, Shuichi Kaneko ${ }^{1}$, Naoto Nagata ${ }^{1}$, Yusuke Ushida ${ }^{2}$, Yudai Aoki ${ }^{2}$ and Hiroyuki \\ Suganuma ${ }^{2}$ \\ ${ }^{1}$ Brain/Liver Interface Medicine Research Center, Kanazawa University, Kanazawa, Japan \\ ${ }^{2}$ KAGOME CO. LTD., Nagoya, Japan
}

\begin{abstract}
Nuclear factor (erythroid-derived 2)-like 2 (Nrf2) is a master regulator of the antioxidant response and plays a role in maintaining glucose homeostasis. Although synthetic Nrf2 inducers alleviate obesity in animal models, they are not clinically available due to safety concerns. We examined the effect of glucoraphanin (GR), a naturally occurring Nrf2 inducer, on high-fat $\operatorname{diet}(\mathrm{HFD})$-induced obesity and insulin resistance in mice. Eight-week-old male C57BL/6J mice were fed a HFD or a HFD containing GR (300 mg/kg) for 14 weeks. GR suppressed HFD-induced weight gain by $15 \%$ (p < 0.01) without affecting food intake. Indirect calorimetry revealed that GR increased energy expenditure of mice fed HFD by 12\% ( $p<0.01$ ). GR improved glucose tolerance, hyperinsulinemia, and fatty liver and enhanced hepatic insulin signaling assessed by phospho-Akt. A flow cytometry analysis revealed that GR decreased the number of liver macrophages identified as CD45+CD11b+F4/80+ cells in mice fed a HFD. In addition, HFD-GR mice had 67\% fewer CD11c+CD206- (M1) macrophages but 20\% more CD11c-CD206+ (M2) macrophages compared with HFD mice, resulting in a predominance of the M2 compared to the M1 macrophage population in the liver. Although GR did not affect accumulation of adipose tissue macrophages (ATMs), it caused an M2 dominant phenotypic shift of ATMs. Importantly, the weight-reducing and insulin-sensitizing effects of GR were abolished in HFD-fed Nrf2 knockout mice. Thus, GR-mediated activation of Nrf2 relieved obesity, and alternatively activated macrophages in the liver and adipose tissue, thereby attenuating obesity-related inflammation and insulin resistance in mice.
\end{abstract}

\section{Barbaloin, A Compound Derived from Aloe vera, Inhibits Adipogenesis in 3T3-L1 Adipocytes via AMPK- $\alpha$ Activation}

\author{
Dong-Hyun Youn ${ }^{1 *}$, JongWook Kang ${ }^{1}$, Yunu Jung ${ }^{1}$, Jinbong Park ${ }^{1}$, Hye-Lin Kim ${ }^{1}$, Mi-Young Jeong ${ }^{1}$, Jae-Young Um ${ }^{1}, J^{-}$-Ye \\ $\mathrm{Kee}^{2}$ and Seung-Heon Hong ${ }^{2}$ \\ ${ }^{1}$ Department of Pharmacy, College of Korean Medicine, Institute of Korean Medicine, Kyung Hee University, 26 Kyungheedae-ro, \\ Dongdaemun-Gu, Seoul, Republic of Korea \\ ${ }^{2}$ Department of Oriental Pharmacy, College of Pharmacy, Wonkwang University, 460 Iksandae-ro, Iksan, Jeonbuk, Republic of Korea
}

\section{Abstract}

Introduction: Obesity became a major health threat in developed countries recently. However, current medications for obesity are limited because of their adverse effects. Thus, interest in natural products for the treatment of obesity is rapidly growing. Barbaloin is a $\mathrm{C}$-glucoside of aloe emodinanthrone which is found in the plant Aloe vera. This study examined the anti-obesity effect of barbarloin by using 3T3-L1 adipocytes.

Methods: The cellular lipid content in 3T3-L1 adipocytes was assessed by Oil Red O staining. Expression of peroxisome proliferators activated receptor- $\gamma(\mathrm{PPAR}-\gamma)$ and CCAAT/enhancer-binding protein- $\alpha(\mathrm{C} / \mathrm{EBP}-\alpha)$ was determined by realtime RT-PCR analyses. In order to examine changes on AMP-activated protein kinase- $\alpha$ (AMPK- $\alpha$ ), immunoblot assays were performed with protein extract from cells by western blotting.

Results: Barbaloin suppressed lipid accumulation in the process of differentiation of 3T3-L1 adipocytes. During the differentiation of 3T3-L1 adipocytes, barbaloin treatment significantly down regulated the mRNA expressions of PPAR- $\gamma$ and $\mathrm{C} / \mathrm{EBP}-\alpha$. It also significantly up-regulated the phosphorylation of AMPK- $\alpha$, when examined by a western blot assay. These results indicate that Barbaloin has a potent antiadipogenic effect in 3T3-L1 cells due to the inhibition of adipocyte differentiation and adipogenesis.

Conclusion: With the continuing spread of obesity prevention as a fundamental medicine strategy, both clinicians and researchers should take a closer look at herbal medicine. Our results suggest barbaloin, which falls in this category as it is derived from a natural plant, may be beneficial in the treatment of obesity and can be used as a safe natural promoter of health. 


\title{
Secoisolariciresinol Diglucoside Improves High-Fat Diet-induced Obesity in Mice by Activating UCP1 in Brown and White Adipose Tissues
}

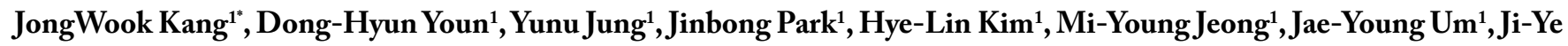 \\ $\mathrm{Kee}^{2}$ and Seung-Heon Hong ${ }^{2}$ \\ ${ }^{1}$ Department of Pharmacy, College of Korean Medicine, Institute of Korean Medicine, Kyung Hee University, 26 Kyungheedae-ro, \\ Dongdaemun-Gu, Seoul, Republic of Korea \\ ${ }^{2}$ Department of Oriental Pharmacy, College of Pharmacy, Wonkwang University, 460 Iksandae-ro, Iksan, Jeonbuk, Republic of Korea
}

\begin{abstract}
Introduction: Obesity is a metabolic disorder characterized by chronic inflammation and dyslipidemia and is a strong predictor for the development of hypertension, diabetes mellitus, and cardiovascular disease. Flaxseeds are used for treating metabolic diseases such as obesity in Traditional Korean Medicine. Secoisolariciresinol diglucoside (SDG) is a main substance of lignan which exists abundantly in flaxseeds. This study examined anti-obesity functions of SDG by assessing changes in highfat diet (HFD)-induced obese C57BL/6 mice and the factors related to adipogenesis.
\end{abstract}

Methods: We induced obesity in C57BL/6 mice by HFD administration for 12 weeks. The body weight and fat tissue weights were measured. Expressions of uncoupling protein1 (UCP1), Peroxisome proliferator-activated receptor gamma coactivator 1-alpha (PGC-1 $\alpha$ ) and cell death-inducing DFFA-like effector a (Cidea) in the adipose tissues were determined by real-time RT-PCR and western blotting assays.

Results: SDG reduced body weight, size and mass of adipose tissues. UCP1, PGC1, and cidea were up-regulated in the interscapular brown adipose tissue (iBAT) and inguinal white adipose tissue (iWAT). These results demonstrate that SDG has a potent anti-obesity effect in vivo, due to the induction of iBAT activation and beige adipocyte recruitment.

Conclusion: Due to the limits of currently available drugs, the necessity for new drugs for the treatment of obesity is rapidly growing. Our results suggest that SDG, the nature-derived agent, shows anti-obesity effects by activating iBAT and inducing "browning" in iWAT. Therefore, SDG shows a new potential pharmacotherapy as a thermogenic agent in the treatment of obesity.

\section{E3 Ubiquitin Ligase Synoviolin Inhibitors has Effects on Obesity}

\author{
Satoko Aratani ${ }^{1-3^{*}}$, Hidetoshi Fujita ${ }^{1,2}$, Maho Yokota ${ }^{1,4}$, Kusuki Nishioka ${ }^{1}$, Toshihiro Nakajima ${ }^{1,2,5,6}$ and Naoko Yagishita $^{4}$ \\ ${ }^{1}$ Institute of Medical Science, Tokyo Medical University, Japan \\ ${ }^{2}$ Department of Future Medical Science, Tokyo Medical University, Japan \\ ${ }^{3}$ Physician, Student and Researcher Support Center, Tokyo Medical University, Japan \\ ${ }^{4}$ Tokyo University of Pharmacy and Life Science, Japan \\ 5integrated Gene Editing Section (iGES), Tokyo Medical University Hospital, Japan \\ ${ }^{6}$ Bay Side Misato Medical Center, Japan \\ ${ }^{7}$ Institute of Medical Science, St. Marianna University School of Medicine, Japan
}

\begin{abstract}
We cloned an E3 ubiquitin ligase synoviolin (SYVN1) from synoviocytes of rheumatoid arthritis (RA) patients. SYVN1 is involved in endoplasmic reticulum associated degradation (ERAD) and mediates preferentially the degradation of secreted proteins. We reported that SYVN1 has crucial roles on proliferation of synoviocytes, fibrosis and arthritis (Genes Dev., EMBO J, Nat. Clin. Pract. Rheumatol.). Since SYVN1 is a cytoplasmic enzyme and is likely related to several disorders, therefore we carried out various kinds of high through put screening and identified several classes of chemical compounds which inhibit SYVN1 ubiquitination activity. These inhibitors suppress synoviocytes proliferation in patients with RA, and reduce the occurrence of arthritis in an RA model mouse (Int. J. Mol. Med.). Furthermore, we established the post-neonatal SYVN1knockout mice induced by tamoxifen administration. Analysis of the knockout mice has indicated that SYVN1 is associated with energy metabolism and mitochondrial biosynthesis through peroxisome proliferator-activated receptor gamma, coactivator $1 \beta$ (PGC-1 $\beta$ ) degradation (EMBO J). To examine the effects of SYVN1 inhibitors on obesity, we daily administrated the inhibitors to mice intraperitoneally. They reduced body weight and white adipose tissue. These results indicated that SYVN1 may represent a potential therapeutic target for obesity.
\end{abstract}




\title{
Third-Degree Family Health History and Perception of Disease Risk
}

\author{
Liana F Romero, Vasileios Margaritis and Aaron Mendelsohn \\ Walden University, MN, USA
}

\begin{abstract}
Chronic diseases are a significant cause of illness and mortality in the United States. Hereditary predisposition to chronic diseases is a useful indicator for identifying people at risk for disease development. An ideal tool for determining this predisposition is the CDC, NIH, and AAFP recommended third-degree Family Health History (FHH). The aim of this quantitative, cross-sectional study based on the theoretical frameworks of social constructivism and the health belief model was to assess the possible influence between the completed third-degree FHH and the participant's perception of disease risk. Twohundred seventy-three participants were recruited from health care facilities and from the general population using convenience sampling. A statistically significant association between the presence of heart disease, stroke, breast cancer, ovarian/cervical cancer, prostate cancer, colon cancer, and diabetes and the perception of risk for the particular disease was noted. A familial history of stroke appeared to be the strongest predictor of perception of disease risk. Moreover, increasing age, particularly within the age range of 40 to 57, was associated with increasing levels of perception of disease risk for heart disease, stroke, and prostate cancer. Individuals from the general population significantly indicated higher than average risk for colon cancer compared to those from health care facilities. Social change implication of this study may be the widespread implementation of a familial health history questionnaire that leads to an impactful, higher degree of disease risk awareness, prompting preventive action on the part of the individual, and leading to improved individual and population health.
\end{abstract}

\section{Body Mass Index and Suicide Behavior in Chinese Adolescents}

\author{
Cun-Xian Jia ${ }^{1,2^{*}}$ and Hua Chen ${ }^{1,2}$ \\ ${ }^{1}$ Department of Epidemiology, Shandong University School of Public Health, China \\ ${ }^{2}$ Center for Suicide Prevention Research, Shandong University, China
}

\begin{abstract}
Objectives: This study aims to test whether there is association between BMI and suicide behavior in Chinese adolescents.

Methods: A questionnaire survey of 2,090 adolescents (mean age 15.5 years, range: 8-21 years, 49.1\% males) was conducted in 3 high schools in 2007, Lijin county, Shandong, China. All participants were categorized into three BMI categories (underweight, healthy weight, and overweight/obese) according to the cut point of the latest Chinese criteria. Multivariate logistic regression was performed to examine the association between BMI and suicide behavior. All data analyses were conducted with SPSS (version 18.0).

Results: The overall prevalence in the past year of suicidal ideation, suicidal plan and attempted suicide were $6.2 \%, 2.7 \%$ and $1.7 \%$, respectively. Overall, $10.5 \%$ of the samples were underweight, $79.5 \%$ were normal weight and $10 \%$ were overweight/ obese according to their BMI. The prevalence of suicidal ideation, suicidal plan and attempted suicide were 5.5\%, 1.4\%, and $0.9 \%$ in the underweight group; $6.3 \%, 3.1 \%$, and $1.9 \%$ in the normal weight group; $5.7 \%, 1.0 \%$ and $1.4 \%$ in the overweight/ obese group. After adjusting for age, numbers of friend, negative life events, smoking, alcohol use, health status, Internalizing, Externalizing and compared with normal weight, ORs (95\% CIs) of underweight to suicidal ideation, suicidal plan, attempted suicide were $0.81(0.43-1.53), 0.40(0.12-1.33), 0.45(0.10-1.92)$; of overweight/obese were $0.88(0.47-1.68), 0.29(0.07-1.24)$, and $0.73(0.22-2.45)$.
\end{abstract}

Conclusion: There was no significant association between BMI and suicide behavior in adolescents.

\section{Noscapinoids: New Cancer Chemotherapeutic}

\author{
Vartika Tomar $^{1,2^{*}}$ and Ramesh Chandra ${ }^{1,2}$ \\ ${ }^{1}$ Department of Chemistry, University of Delhi, India \\ ${ }^{2}$ Dr. B. R. Ambedkar Center for Biomedical Research, University of Delhi, India
}

\section{Abstract}

Cancer is linked to smoking, alcohol consumption, diet, obesity and physical inactivity, chronic infections (viruses, bacteria, 
parasites), genetic susceptibility, occupational exposures, sunlight, radiation, medical and iatrogenic factors, environmental pollution, and reproductive factors. Recurrent cancers often become resistant. Noscapine however remains effective in some such instances, e.g., taxane-resistant ovarian cancer. Noscapine and analogs also do not show signs of neurotoxicity or immunosuppression. On the other hand, noscapine was shown to be neuroprotective in mouse models of neurodegenerative disease and in stroke patients. Like low doses of colchicine, noscapine and its analog also show anti-inflammatory activities. There are indications of a preventive use for noscapine in ischemia-reperfusion injury and fibrosis. The entire biosynthetic pathway of noscapine is encoded as gene cluster within 401 kilo bases of genomic DNA, opening up opportunities for the large-scale biotechnological production of noscapine for medicinal needs. Thus, noscapine and its derivatives (noscapinoids) might be cost-effective and safe components of cancer chemotherapeutics. Because of its low toxicity it also might be useful for preventive use in high-risk situations. Four C-9 substituted analogs of noscapine, compound 2-5 have been synthesized and tested in vitro for antiproliferative activity against human glioblastoma cancer cell line, U87. All compounds obtained exhibited antiproliferative activity. The most active compounds 2 and 4 exhibited IC50 values between 6 to $17 \mu \mathrm{M}$ compared to the parent molecule, noscapine, 1 . Thus, we provided sufficient evidence that the substitution on the C-9 position of phenyl ring with bromo and aldehyde groups in noscapine leads to considerable increase in cytotoxicity and may be used as potential anticancer agents.

\title{
Body Composition in Male and Female Mexican Adults with and without Down Syndrome Using a Four-Compartment Model: Prediction Equation Development
}

\author{
Mauro E. Valencia ${ }^{1,2^{*}}$, Luz A. Caraveo ${ }^{1,2}$, Maria del Carmen Candia-Plata ${ }^{1,2}$, Heliodoro Aleman-Mateo ${ }^{1,2}$, Rosa Consuelo \\ Villegas-Valle $^{1,2}$, Lesley Evelyn Antunez-Roman ${ }^{1,2}$ and Herminia Mendivil-Alvarado, ${ }^{1,2}$
}

${ }^{1}$ Department of Nutrition and Metabolism, Centro de Investigación en Alimentacion y Desarrollo, A. C. Hermosillo, Sonora, Mexico

${ }^{2}$ Departments of Chemical and Biological Sciences and Medicine and Health Sciences, University of Sonora, Mexico

\section{Abstract}

Background: Patients with Down syndrome (DS) show reduced bone mineral density (BMD) and bone mineral mass (BMM) that could affect the density of fat free mass (DbFFM) when using 2 compartment (2-C) models.

Objective: To characterize body composition (BC) of individuals with DS and their controls using a Four compartment (4-C) model and to develop BC prediction algorithms.

Methods: One hundred and twenty males and females (60 with DS and 60 controls) matched by weight were recruited from the general population in Hermosillo Sonora, Mexico. The 4-C model (Selinger et al, 1977) was obtained by measuring body density (Db), (BOD POD), bone mineral mass (DXA), total body water estimated by (BIA), based on deuterium dilution (Schoeller et al, 1986) and DbFFM by Wang et al, (2003).

Results: Mean weight for DS and controls was $62.6 \mathrm{~kg}$. Means \pm SD of BMI and height, were: $26.0 \pm 11.6 .0$ vs. $24.2 \pm$ $10.1(\mathrm{p}=0.171) \mathrm{kg} / \mathrm{m}^{2}$ and $147 \pm 8.5$ vs. $163 \pm 6.4 \mathrm{~cm}(\mathrm{p}<0.0001)$, respectively. Body fat (4-C), BMM and DbFFM were: 31.4 \pm 11.5 vs. $24 \pm 10.5 \%(\mathrm{p}<0.0001) ; 1.640 \pm 0.27 \mathrm{vs.} 2.076 \pm 0.31 \mathrm{~kg}(\mathrm{p}<0.0001)$ and $1.0820 \pm 0.003 \mathrm{vs} .1 .0905 \pm 0.004 \mathrm{~g} / \mathrm{cm}^{3}$ $(\mathrm{p}<0.0001)$, for DS and controls respectively. The 4-C model equation was: FFM kg $=12.324-0.1278^{*} \mathrm{Age}+0.5487^{*}(\mathrm{Height} 2 / \mathrm{R})$ + 0.1548*Weight + 3.019*Sex (R2 = 0.98; SRMSE = 1.25), where: Age (years); Weight (kg); Height (cm); R (ohms); and Sex (males $=1$; females $=0$ ). Predicted FFM did not differ from the mean measured value by the 4-C model, was accurate, precise and had no significant bias.

Conclusion: Due to the differences in BMM and DbFFM, 2-C models might not be suitable for measuring body composition in adults with Down syndrome.

\section{Consumption of Fat, Oil Intake and Percent of Body Fat in Mongolian People}

\author{
Ouynbileg Janchiv ${ }^{1}$, Ichinkhorloo Bonduush ${ }^{1}$, Enkhtuya Palam ${ }^{1}$, Bolormaa Norov ${ }^{1}$, Tserendolgor Uush ${ }^{1}$, Tsogtbaatar \\ Byambaa $^{1}$ and Otgontuya Dugee ${ }^{2}$
}

${ }^{1}$ Public Health Institute of Mongolia, Mongolia

${ }^{2}$ Monash University of Australia, Australia

\section{Abstract}

Type of oil used for cooking, $77.1 \%$ (95\% CI, 70.3-83.9) of the survey respondents reported using vegetable oil, $17.1 \%(95 \%$ 
CI, 10.8-23.3) - animal fat, 5.5\% (95\% CI, 3.3-7.8) - other types of fats and oils and 0.3\% (95\% CI, 0.1-0.5) did not use fat or oil for cooking. Of male respondents, 73.7\% (95\% CI, 65.9-81.5) used vegetable oil, 20.3\% (95\% CI, 12.8-27.9) - animal fat, 5.5\% (95\% CI, 3.2-7.8) - other oil and 0.4\% reported no use of fat or oil in cooking. 94.1\% (95\% CI, 91.5-96.7) of urban and $60.7 \%$ (95\% CI, 48.6-72.7) of rural respondents used vegetable oil. Body fat: Mean body fat of men aged 15 - 64 years was above the reference value or $22.2 \%$ (95\% CI 21.4-23.0), while for women was within normal range or 29.3\% (95\% CI 28.5-30.2). Men aged 25 and above, and women aged 35 and above had mean body fat content above reference values, i.e. were obese. In 45-64 age group one in four men and one in three women had increased body fat content, i.e. were obese. There was no significant difference in mean body fat \% between urban and rural survey participants. Proportion of men with very high body fat was $34.3 \%$ (95\% CI 28.8-39.8) in urban, and 33.8\% (95\% CI 27.9-39.6) in rural areas. Similarly, the prevalence of very high body fat or obesity in 15-64 year-old women was not significantly different in urban vs. rural areas or 26.9\% (95\% CI 22.7-31.1) and 23.4\% (95\% CI 19.3-27.4), respectively. Body fat \% was high or very high in 43\% of men and 52.2\% of women. Body fat \% tended to increase with age irrespective of gender.

\section{Submit Your Next Manuscript}

We are currently accepting submissions from new and published authors. Read our inaugural issue articles and submit your next manuscript to Journal of Obesity and Chronic Diseases (JOCD).

Submit Online: http://www.editorialmanager.com/usg

Citation: Proceedings of the Inaugural International Conference on Obesity and Chronic Diseases (ICOCD-2016). $J$ Obes Chronic Dis 1(Suppl 1): S1-S49.

Copyright: This is an Open Access article distributed under the terms of the Creative Commons Attribution 4.0 International License (CC-BY) (http://creativecommons.org/licenses/by/4.0/) which permits commercial use, including reproduction, adaptation, and distribution of the article provided the original author and source are credited. Published by United Scientific Group.

Received: September 15, 2016 Accepted: October 19,2016 Published: October 21, 2016 